USE OF GENETIC ALGORITHM FOR SELECTION OF REGULARIZATION PARAMETERS IN MULTIPLE CONSTRAINT INVERSE ECG PROBLEM

A THESIS SUBMITTED TO

THE GRADUATE SCHOOL OF NATURAL AND APPLIED SCIENCES OF

MIDDLE EAST TECHNICAL UNIVERSITY

BY

\begin{abstract}
ALIREZA MAZLOUMI GAVGANI
IN PARTIAL FULFILLMENT FOR THE REQUIREMENTS

FOR

THE DEGREE OF MASTER OF SCIENCE

IN

ELECTRICAL AND ELECTRONICS ENGINEERING
\end{abstract}


Approval of the thesis:

\section{USE OF GENETIC ALGORITHM FOR SELECTION OF REGULARIZATION PARAMETERS IN MULTIPLE CONSTRAINT INVERSE ECG PROBLEM}

Submitted by ALIREZA MAZLOUMI GAVGANI in partial fulfillment of the requirements for the degree of Master of Science in Electrical and Electronics Engineering Department, Middle East Technical University by,

Prof. Dr. Canan Özgen

Dean, Graduate School of Natural and Applied Sciences

Prof. Dr. İsmet Erkmen

Head of Department, Electrical and Electronics Engineering

Assist. Prof. Dr.Yeşim Serinağaoğlu Doğrusöz

Supervisor, Electrical and Electronics Engineering Dept., METU

\section{Examining Committee Members:}

Prof. Dr. Nevzat G. Gençer

Electrical and Electronics Engineering Dept., METU

Assist. Prof. Dr.Yeşim Serinağaoğlu Doğrusöz

Electrical and Electronics Engineering Dept., METU

Prof. Dr. B. Murat Eyüboğlu

Electrical and Electronics Engineering Dept., METU

Assist. Prof. Dr. İlkay Ulusoy

Electrical and Electronics Engineering Dept.,

Dr. Özlem Birgül

TÜBİTAK, UZAY

Date: 28.01.2011 
I hereby declare that all information in this document has been obtained and presented in accordance with academic rules and ethical conduct. I also declare that, as required by these rules and conduct, I have fully cited and referenced all material and results that are not original to this work.

Name, Last Name: Alireza Mazloumi Gavgani

Signature: 


\begin{abstract}
USE OF GENETIC ALGORITHM FOR SELECTION OF REGULARIZATION

PARAMETERS IN MULTIPLE CONSTRAINT INVERSE ECG PROBLEM
\end{abstract}

\author{
Mazloumi Gavgani, Alireza \\ M.Sc. Department of Electrical and Electronics Engineering \\ Supervisor : Assist. Prof. Dr.Yeşim Serinağaoğlu Doğrusöz
}

January 2011, 104 Pages

The main goal in inverse and forward problems of electrocardiography (ECG) is to better understand the electrical activity of the heart. In the forward problem of ECG, one obtains the body surface potential (BSP) distribution (i.e., the measurements) when the electrical sources in the heart are assumed to be known. The result is a mathematical model that relates the sources to the measurements. In the inverse problem of ECG, the unknown cardiac electrical sources are estimated from the BSP measurements and the mathematical model of the torso. Inverse problem of ECG is an ill-posed problem, and regularization should be applied in order to obtain a good solution. Tikhonov regularization is a well-known method, which introduces a tradeoff between how well the solution fits the measurements and how well the constraints on the solution are satisfied. This trade-off is controlled by a regularization parameter, which can be easily calculated by the L-curve method. It is theoretically possible to include more than one constraint in the cost function; however finding more than one regularization parameter to use with each constraint is a challenging problem. It is the aim of this thesis to use genetic algorithm (GA) optimization method to obtain regularization parameters to solve the inverse ECG problem when multiple constraints are used for regularization. The results are presented when there are two spatial constraints, when there is one spatial, one temporal constraint, and when there are two spatial one temporal constraints; the 
performances of these three applications are compared to Tikhonov regularization results and to each other. As a conlcusion, it is possible to obtain correct regularization parameters using the GA method, and using more than one constraints yields improvements in the results.

Keywords: inverse ECG problem, genetic algorithm, regularization 


\title{
$\ddot{O} \mathbf{Z}$
}

\section{ÇOK KISITLI TERS EKG PROBLEMINNDE DÜZENLİLEŞTİRME PARAMETRELERININ SEÇIMINDE GENETIKK ALGORITTMA KULLANILMASI}

\author{
Mazloumi Gavgani, Alireza \\ Yüksek Lisans, Elektrik ve Elektroniks Bölümü \\ Tez Yöneticisi : Yrd.Doç.Yeşim Serinağaoğlu Doğrusöz
}

Ocak 2011, 104 Sayfa

İleri ve geri elektrokardiyografi (EKG) problemlerinde ana hedef kalbin elektriksel aktivitesinin daha iyi bir şekilde anlaşılmasıdır. İleri EKG probleminde, kalpteki elektriksel kaynakların biliniyor olduğu varsayılarak vücut yüzeyi potansiyel (VYP) dağılımı (ölçümler) elde edilmektedir. Sonuç, kaynakları ölçümlerle ilişkilendiren, gövdenin matematiksel bir modelidir. Geri EKG probleminde ise, VYP ölçümleri ve ileri problem çözümünden gelen gövdenin matematiksel modeli kullanılarak elektriksel kaynaklar kestirilmektedir. Geri EKG problemi kötü konumlanmış bir problemdir ve güvenilir bir sonuç elde edebilmek için düzenlileştirme uygulanması gerekmektedir. $\mathrm{Bu}$ alanda en çok kullanılan yöntem Tikhonov düzenlileştirme metodudur. Bu yöntem, çözümün gerçek değerlerine yakınlığı ve çözümün göstermesi beklenen özellikler (kısıtlar) arasında bir ödünleşim gerçekleştirir. $\mathrm{Bu}$ ödünleşim, L-eğrisi (L-curve) yöntemiyle kolaylıkla hesaplanan bir düzenlileştirme parametresiyle kontrol edilebilmektedir. Teorik olarak maliyet fonksiyonunun birden fazla kısıt içermesi sağlanabilir. Fakat bu kısıtların her biri için ayrı bir düzenlileştirme parametresinin bulunması problem oluşturmaktadır. Bu tezin amacı, geri EKG problemini birden fazla kısıt kullanıldığı durumda çözmek için genetik 
algoritma (GA) optimizasyonu kullanarak düzenlileştirme parametrelerini hesaplamaktır. Sonuçlar iki uzamsal kısıt, bir uzamsal bir de zamansal kısıt, ve iki uzamsal bir zamansal kısıt olduğu durumlar için elde edilmiştir. Bu sonuçlar, Tikhonov düzenlileştirmesi sonuçları ve birbirleriyle karşılaştırılmışlardır. Sonuç olarak, GA yöntemi ile doğru düzenlileştirme parametreleri bulounabilmektedir, ve birden fazla kısıt kullanmak çözümde iyileşmelerin olmasını sağlamaktadır.

Anahtar kelimeler: ters EKG problemi, genetic algoritma, düzenlileştirme 
To my family and friends... 


\section{ACKNOWLEDGEMENT}

I would like to thank my thesis supervisor Prof. Dr.Yeşim Serinağaoğlu Doğrusöz for providing me this research opportunity and guiding me through the study.

This study is part of the project 105E070 that is supported by Turkish Scientific and Technological Research Council (TUBITAK).

I would like to express my thanks and gratitude to the members of the jury specially Prof. Dr. B. Murat Eyüboğlu and Prof. Dr. Nevzat G. GENÇER.

I would like to thank all my colleagues and friends in the ECG, MRI and Brain Research Laboratories specially Umit Aydin, Ceren Bora, Ersin karci, Kerem Kara, Evren Degirmenci, Emre Arpinar, Ali Ersoz , Rasim Boyacioglu, Alper cevik, Soner keceli, Gorkem Kandemir, Cihan Goksu, Tankut Topal, Feza Carlak, Bulkar Erdogan, Berna Akici, Reyhan Zengin , Azadeh Kamali for their comments and technical supports.

I also would like to express my appreciation to all my friends especially Faith Nar, Sona Khaneshenas, Maryam Farzin, Samira Ghoshouni, Yousef Zamani, Aslan Eslami, Aydin Tahmasebifar, Sina Mojtehedi, Adnan mousvani for their support throughout the development of this thesis.

I like to express very special thanks to my dear family for their endless support and love throughout my education.

And finally I would like to dedicate this work to my beloved father which I miss very much, and I would have loved for him to see this work. 


\section{TABLE OF CONTENTS}

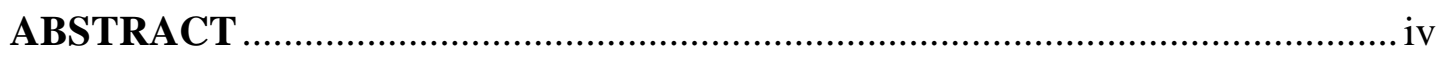

ÖZ

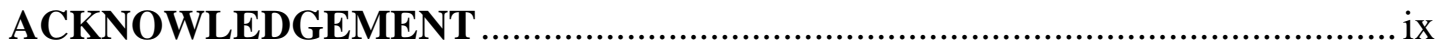

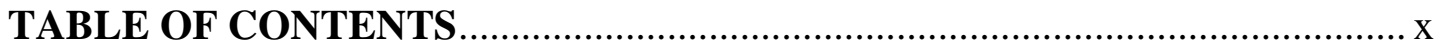

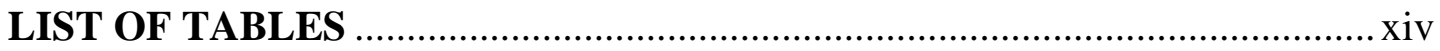

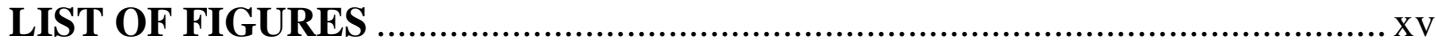

\section{CHAPTERS}

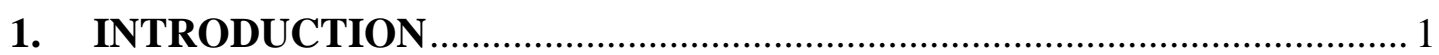

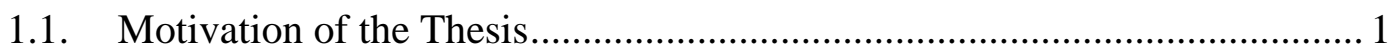

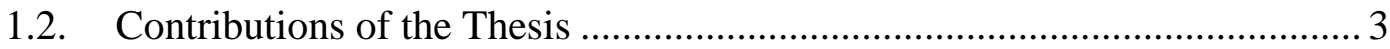

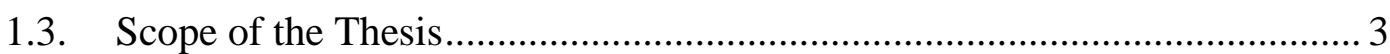

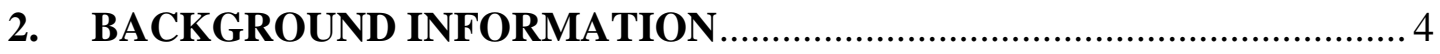

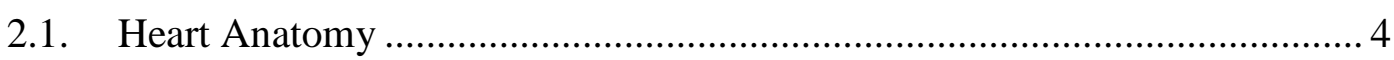

2.1.1. Cardiac Chambers ............................................................................ 5

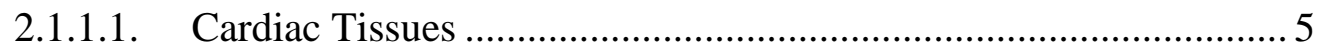

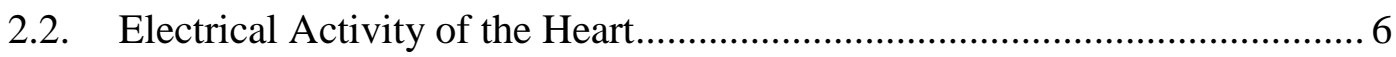

2.2.1. Action Potential Generation .............................................................. 6

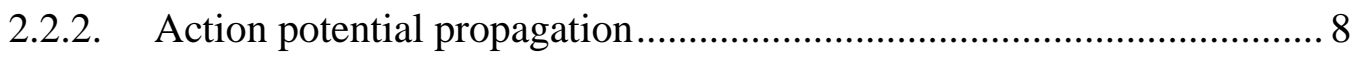

2.2.3. Electrical Conduction System of the Heart ......................................... 9

2.3. Standard 12 Lead Electrocardiography (ECG)........................................ 9

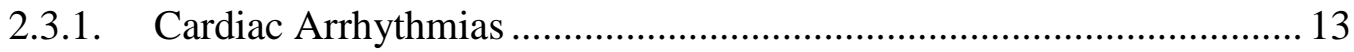


2.3.1.1. Cardiac Re-entry Phenomenon ............................................... 13

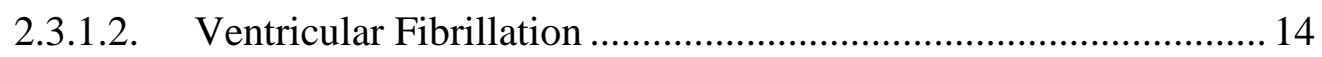

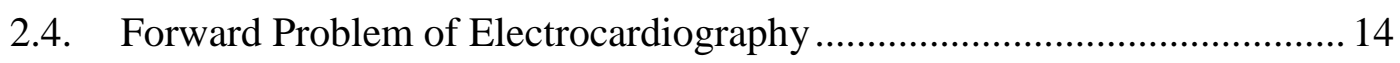

2.4.1. Geometric Information ................................................................. 14

2.4.2. Numeric Methods for Solving the Forward Problem........................... 15

2.5. Inverse Problem of Electrocardiography.............................................. 16

2.5.1. Introduction to Inverse Problem of Electrocardiography.................... 16

2.5.2. Methods in the Literature to Solve the Inverse ECG Problem......... 18

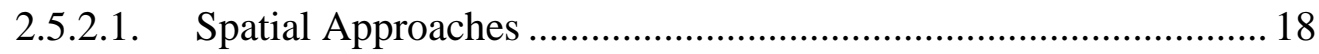

2.5.2.2. Spatial Temporal Approaches................................................. 20

2.5.3. Using Genetic Algorithm in Inverse ECG Problem............................ 21

2.5.4. Other Approaches to Obtain Cardiac Electrical Activity.................... 21

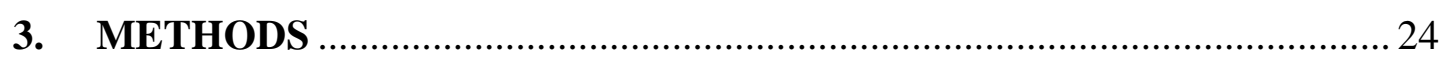

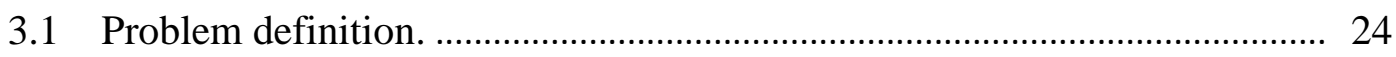

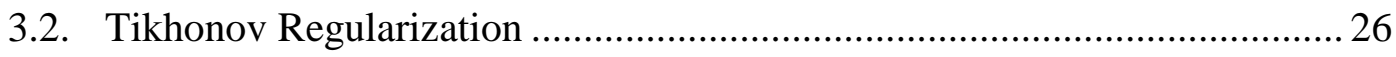

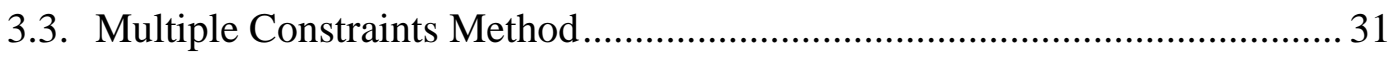

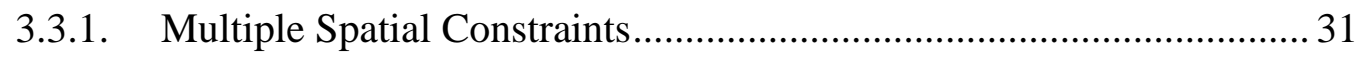

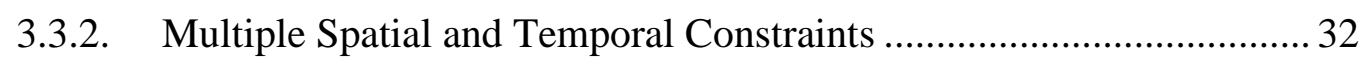

3.4. Regularization parameter calculation .................................................. 34

3.4.1. Regularization Parameter Calculation Using Real Data (Maximum CC

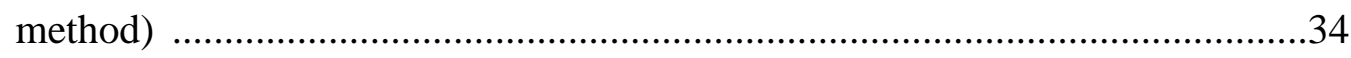

3.4.2. Regularization Parameters Calculation Using Genetic Algorithm ...... 35

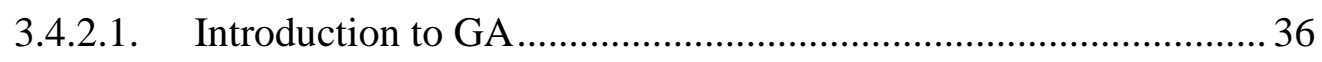

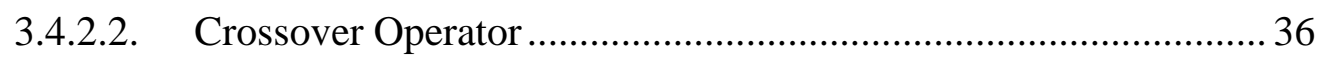




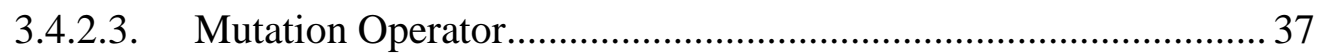

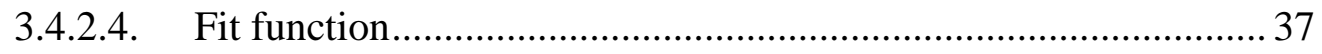

3.4.3. Using GA for Regularization Parameter Selection ............................. 38

3.4.3.1. Fit Function for Regularization Parameter Calculation ................ 40

3.4.3.2. Real Valued Crossover................................................................ 41

3.4.3.3. Real Valued Mutation .............................................................. 42

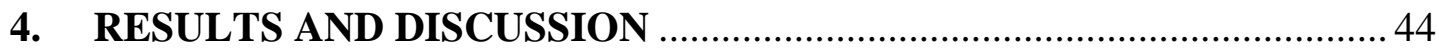

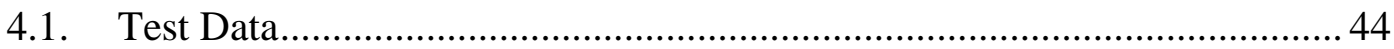

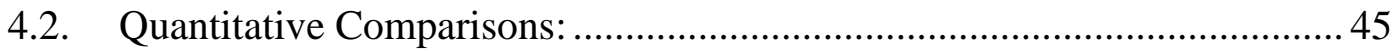

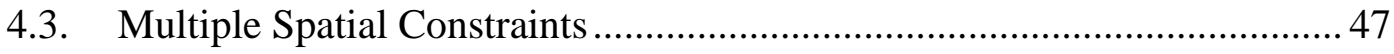

4.3.1. Two Spatial Constraints with $30 \mathrm{~dB}$ Noise Data .............................. 48

4.3.2. Two Spatial Constraints with $10 \mathrm{~dB}$ Noise Data ............................... 56

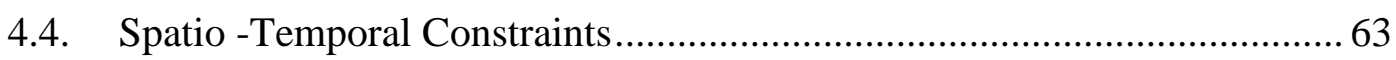

4.4.1. One Spatial and One Temporal Constraints with $30 \mathrm{~dB}$ Noise Data... 64

4.4.2. One Spatial One Temporal Constraints with $10 \mathrm{~dB}$ Noise Data.......... 70

4.4.3. Two Spatial One Temporal Constraints with $30 \mathrm{~dB}$ Noise Data ......... 77

4.4.4. Two Spatial One Temporal Constraints with $10 \mathrm{~dB}$ Noise Data ......... 79

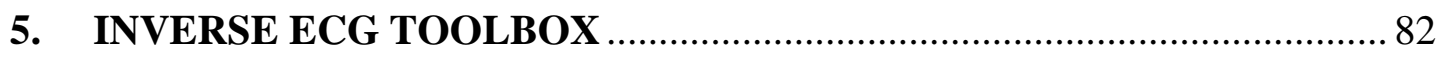

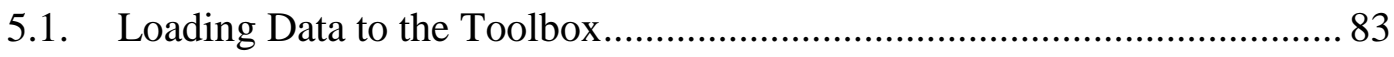

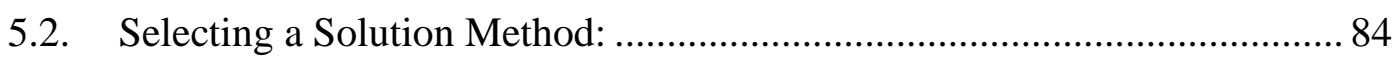

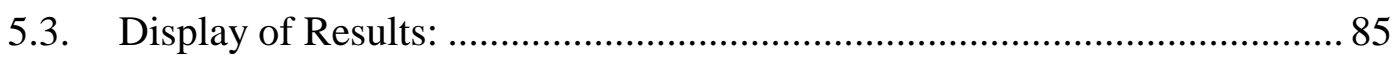

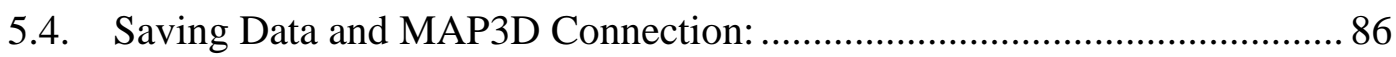

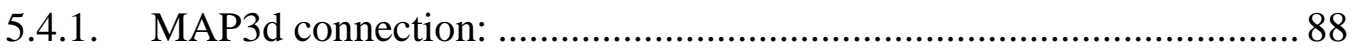

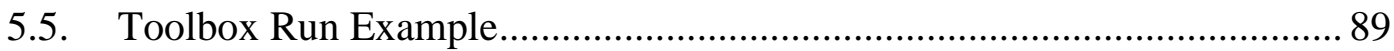


6. CONCLUSION

6.1. Future work 94

REFERENCES 95

\section{APPENDICES}

APPENDIX A 101

USING PRE/POST DIAGONALIZATION METHOD TO SOLVE MULTIPLE

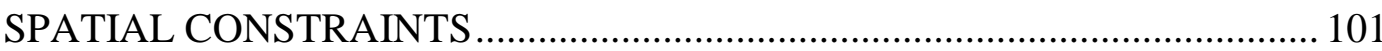

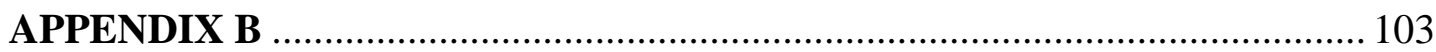
USING PRE/ POST DIGONALIZATION METHOD TO SOLVE MULTIPLE SPATIO-TEMPORAL CONSTRAINTS 103 


\section{LIST OF TABLES}

\section{TABLES}

Table 4.1: MCC and GA method solution results for $30 \mathrm{~dB}$ SNR data.

Table 4.2 : Means and standard deviations of CC and RDMS values for $30 \mathrm{~dB} \ldots . .51$

Table 4.3: Means and standard deviations of CC and RDMS values for second data set

Table 4.4: MCC and GA method solution results for $10 \mathrm{~dB}$ SNR data

Table 4.5: Means and standard deviations of CC and RDMS values .59

Table 4.6 : Means and standard deviations of CC and RDMS values for second data set. 59

Table 4.7 : MCC and GA method solution results for $30 \mathrm{~dB}$ SNR data. 65

Table 4.8: Means and standard deviations of CC and RDMS values 66

Table 4.9: The average and standard deviation values of RDMS and CC of the second data set.

Table 4.10: MCC and GA method solution results for $10 \mathrm{~dB}$ SNR data. .71

Table 4.11 : The average and standard deviation values of RDMS and CC .72

Table 4.12: The average and standard deviation values of RDMS and CC of the second data set .73

Table 4.13: The average and standard deviation values of RDMS and CC 79

Table 4.14: The average and standard deviation values of RDMS and CC .81 


\section{LIST OF FIGURES}

\section{FIGURES}

Figure 2.1: Action potential generated by a tissue in the heart [5] ........................... 7

Figure 2.2: Action potential changes from one region to another [6] ........................ 8

Figure 2.3: 12 lead Electrocardiograph electrode placement[8] ............................. 10

Figure 2.4: A single heart beat detected by Electrocardiograph [3] ......................... 11

Figure 2.5: Cardiac AP conduction path and the corresponding wave on the ECG signal [9].

Figure 2.6: Inverse ECG [14].Using body surface potentials and geometric information to calculate epicardial potentials ........................................................ 17

Figure 3.1.1: Eigen values of transfer matrixATA................................................ 26

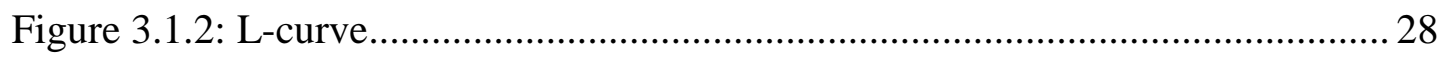

Figure 3.1.3: a) Two Gaussian-shaped peaks (original image), b) Tikhonov solution of the problem. c) Tikhonov solution with a slight change in the regularization

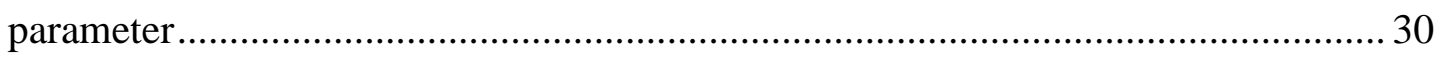

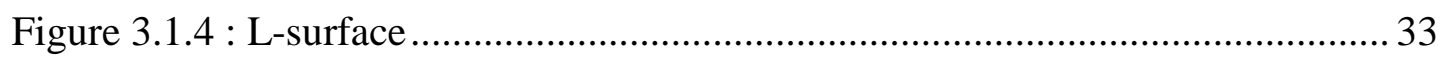

Figure 3.1.5 :Pseudocode in finding the best solution .......................................... 35

Figure 3.1.6: Flow chart of the inputs needed for regularization parameter calculation

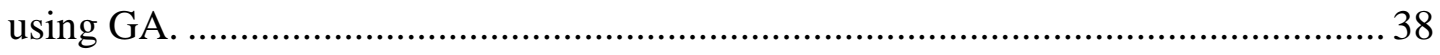

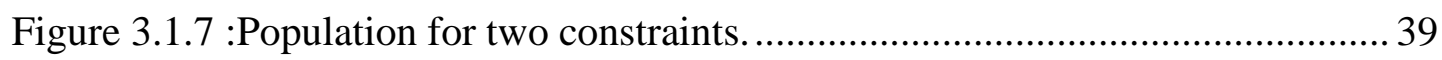

Figure 3.1.8: Father and mother chromosomes.................................................. 41

Figure 3.1.9: child chromosome before crossover .............................................. 41

Figure 3.1.10: child chromosome after crossover............................................... 42

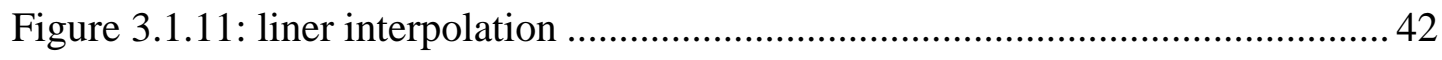

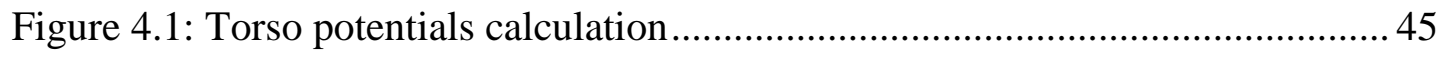

Figure 4.2:Correlation coefficient calculated for all $\lambda$ and $\eta$ values using MCC method.

Figure 4.3: Correlation coefficient figure for each time instant. .49 
Figure 4.4 : RDMS figure for each time instant.

Figure 4.5: Epicardial potential map for 47th time instant. a) Real Epicardial Potentials, b) Tikhonov method, c) Multiple Constraints (MCC), d) Multiple Constraints (GA).

Figure 4.6: Epicardial Potential map for $91^{\text {th }}$ time instant. a) Real Epicardial Potentials, b) Tikhonov method, c) Multiple Constraints (MCC), d) Multiple Constraints (GA).

Figure 4.7: Epicardial potential map for $93^{\text {th }}$ time instant. a) Real Epicardial Potentials, b) Tikhonov method, c) Multiple Constraints (MCC), d) Multiple Constraints (GA).

Figure 4.8 : Epicardial potential map for $6^{\text {th }}$ time instant. a) Real Epicardial Potentials, b) Tikhonov method, c) Multiple Constraints (MCC), d) Multiple Constraints (GA). .55

Figure 4.9: Correlation coefficient calculated for all $\boldsymbol{\lambda}$ and $\boldsymbol{\eta}$ values using MCC method for $10 \mathrm{~dB}$ data. .56

Figure 4.10: Correlation coefficients figures for each time instant. 57

Figure 4.11: RDMS figures for each time instant .58

Figure $4.12: 21^{\text {th }}$ time instant of the epicardial potentials. a) Real Epicardial Potentials, b) Tikhonov method, c) Multiple Constraints (MCC), d) Multiple Constraints (GA).

Figure 4.13: Epicardial potential map $10 \mathrm{~dB}$ SNR (47th time instant). a) Real Epicardial Potentials, b) Tikhonov method, c) Multiple Constraints (MCC), d) Multiple Constraints (GA).

Figure 4.14: Epicardial potential map $10 \mathrm{~dB}$ SNR for93 ${ }^{\text {th }}$ time instant. a) Real Epicardial Potentials, b) Tikhonov method, c) Multiple Constraints (MCC), d) Multiple Constraints (GA)

Figure 4.15: Correlation Coefficient calculated for all $\lambda$ and $\eta$ values using MCC method 64

Figure 4.16 : CC figures for each time instant. 65

Figure 4.17: RDMS figures for each time instant. 66

Figure 4.18 epicardial potentials 6th time instant. a) Real Epicardial Potentials, b) Tikhonov method, c) Multiple Constraints (MCC), d) Multiple Constraints (GA).. 68 
Figure 4.19 : epicardial potential map for 47time instant. a) Real Epicardial Potentials, b) Tikhonov method, c) Multiple Constraints (MCC), d) Multiple Constraints (GA).

Figure 4.20 :Correlation coefficient calculated for all $\lambda$ and $\eta$ values using MCC

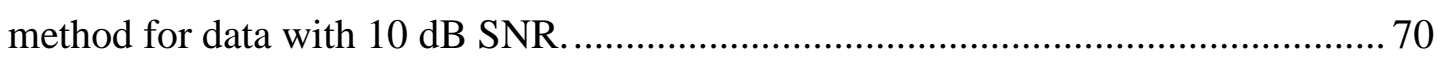

Figure 4.21: Correlation Coefficient figures for $10 \mathrm{~dB}$ SNR data........................... 71

Figure 4.22 : RDMS Figures for 10 dB SNR data ................................................ 72

Figure 4.23 : Epicardial potentials map for 25th time instant. a) Real Epicardial Potentials, b) Tikhonov method, c) Multiple Constraints (MCC), d) Multiple Constraints (GA).

Figure 4.24 : Epicardial potentials map for 47th time instant. a) Real Epicardial Potentials, b) Tikhonov method, c) Multiple Constraints (MCC), d) Multiple Constraints (GA). .75

Figure 4.25 : Epicardial Potential map corresponding to 93th time instant. a) Real Epicardial Potentials, b) Tikhonov method, c) Multiple Constraints (MCC), d)

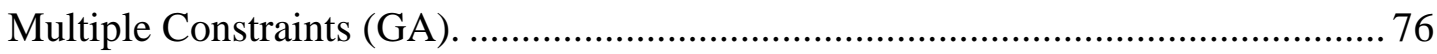

Figure 4.26: Correlation Coefficient figures for $30 \mathrm{~dB}$ SNR data........................... 77

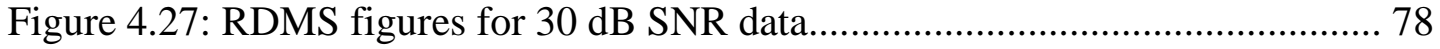

Figure 4.28: Correlation Coefficient figures for $10 \mathrm{~dB}$ SNR data............................79

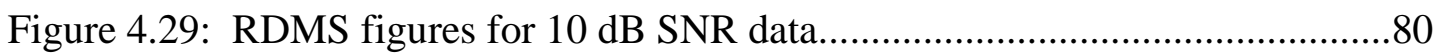

Figure 4.30: epicardial Potential maps corresponding to 85th time instant. a) Real Epicardial Potentials, b) MST (Three Constraints) c) Multiple Constraints (MST), d) Tikhonov

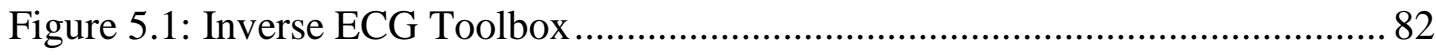

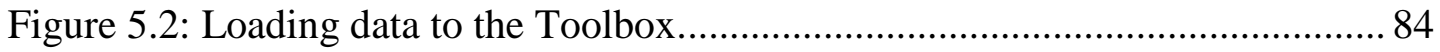

Figure 5.3: Selecting method and inputting corresponding properties.................... 85

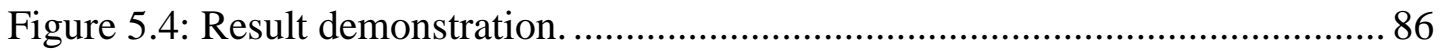

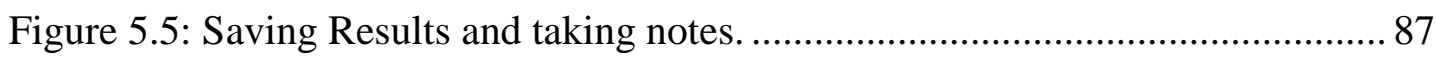

Figure 5.6: Results Display using direct connection to MAP3D .............................. 88

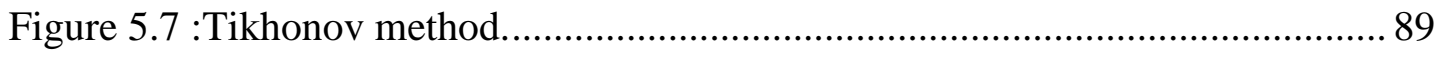

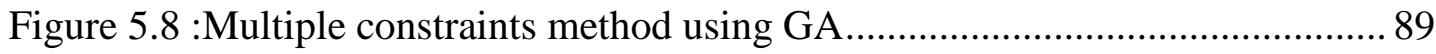




\section{CHAPTER1}

\section{INTRODUCTION}

\subsection{Motivation of the Thesis}

Different diseases cause millions of deaths all over the world every year. With Technological improvement, clinicians have used different equipments instead of surgical operations for determining these diseases. Heart failure affects approximately 15 million people worldwide and since 1968, the role of heart arrhythmias as the primary cause of death has increased fourfold [1]. In The European Union, deaths that annually occur due to cardiac diseases are over 1.9 million [1].

Knowledge about the electrical activity within the heart is essential in order to diagnose arrhythmias. One of the most important medical equipments used for investigating the cardiac disorders such as arrhythmia or myocardial infarction is Electrocardiography (ECG) . It consists of the interpretation of potentials recorded at the body surface (i.e. torso potentials) due to the electrical activity of the heart. Physicians could diagnose certain pathologies with the 12-lead ECG; however low resolution limits this technique's benefits significantly [2].

The 12-lead ECG also suffers from Brody and Respiration, effects and effects of inhomogeneities within the thorax, which cause wrong interpretations [3]. An alternative is to use catheters to image the electrical activity of the heart. This method gives reasonably good results, but the invasive nature of this technique restricts its usage. 
There are other non-invasive techniques to monitor cardiac behavior such as cardiac CT, nuclear imaging, stress electrocardiography and echocardiography. Cardiac source imaging is another non invasive method. Mathematically, cardiac source imaging problem consists of forward and inverse problems. In the forward problem, we calculate the body surface potentials that occur due to equivalent cardiac sources. On the other hand, the estimation of these equivalent cardiac sources from the signals recorded over the body surface is known as the inverse problem of ECG, in which high resolution images of the heart's electrical activity are generated. These images have the potential to support the diagnosis made based on the 12-lead ECG.

While solving the inverse problem, its ill posed nature makes it very difficult to accurately estimate the cardiac sources, and therefore effective imaging is difficult. Many approaches and methods have been proposed to cope with the ill-posedness of the problem. In all of the methods, prior information on the solution is incorporated into the solution. While some approaches have only taken the spatial information on the data, other methods have tried to solve this problem by taking into account both the spatial and temporal aspects of the potential distribution.

In this thesis study, we explored the applicability of incorporating multiple constraints, i.e., multiple kinds of prior information, into the solution, and compared all our solutions to those obtained using Tikhonov Regularization. Tikhonov Regularization is one of the most famous methods used to solve ill-posed inverse problems, in which one constraint is used to regularize the solution. Multiple constraints approach is an extension of Tikhonov regularization method, but instead of a single constraint controlled by single regularization parameter, more than one constraint is included in the cost function with a different regularization parameter controlling the influence of each constraint. In theory, it is easy to extend the cost function to accommodate multiple constraints. However, in practice, one needs to find multiple number of regularization parameters, which is not a straightforward task. In this thesis, we propose an application of Genetic Algorithm (GA) to select the optimum regularization parameters. Although the iterative nature of the GA approach increases the run time, its adaptability to estimate a number of regularization parameters simultaneously makes it an appropriate approach to be used in inverse problem solutions. 


\subsection{Contributions of the Thesis}

A new method based on GA has been proposed in calculating the spatial and/or regularization parameters. Using this method, multiple constraints can be added to the solution of inverse problem to take into account different properties of the solution. To the best of our knowledge, this approach has not been used in inverse ECG applications before.

A graphical user interface has been designed, which could be used for comparing different regularization algorithms, as well as for educational purposes.

\subsection{Scope of the Thesis}

The second chapter in this thesis is devoted to background information about the anatomy and physiology of the heart, the electrical conduction system of the heart; two of the most common heart arrhythmias are also described. We then continue by defining forward and inverse ECG problems. Methods used in the literature to solve these problems will be discussed briefly.

In the third chapter we start by giving the definition of the inverse problem of ECG. Then we explain the Tikhonov regularization method, which is the most popular method in the literature dealing with this problem. We continue by discussing the drawbacks of this method, and the approaches proposed in literature to overcome these drawbacks are discussed. The Genetic Algorithm solution proposed in this work is explained after some introduction about Genetic Algorithm.

The forth chapter of this thesis is devoted to results and discussions. We compare two proposed methods MS and MST with each other and with the Tikhonov solution for two different noise levels. In the fifth chapter Inverse ECG Toolbox designed in this study is introduced and different parts of this toolbox are explained.

In the sixth and final chapter, conclusions derived from this work will be given. 


\section{CHAPTER 2}

\section{BACKGROUND INFORMATION}

Heart failure and heart disabilities cause 15 million deaths worldwide every year [1] while millions of people suffer from cardiac arrhythmias. Therefore, understanding the functioning of the heart is of great importance from both medical and engineering point of views [2]. Thus, in this study we present different aspects of the forward and inverse electrocardiography as well as the solutions that have been discussed in the literature.

In this chapter, first electro-physical aspects of the heart will be discussed; different tissues of heart will be introduced and some major cardiac arrhythmias will be presented. Then, forward problem of electrocardiography will be discussed, followed by the inverse problem of electrocardiography, which is the main subject of this thesis.

\subsection{Heart Anatomy}

The human heart is located in the middle Mediastinum of the thorax; this organ is responsible for pumping blood to all parts of the body. Every single cell in the body is fed by continuous blood flow, which is enriched by nutrition's and oxygen.

Some scientists prefer to call the heart as the electro-mechanic pump of the body. On average, an adult heart beats about 72 times a minute. Hence, in a day (that is, 1,440 minutes), the adult heart beats 103,680 times on average [4]. It is easy to understand the reason why this organ has been given the name of "electro-mechanic pump" by only looking at this number of beats per day. 
Unfortunately, a great number of people worldwide suffer from heart diseases, and millions lose their lives every year due to heart malfunctions. This brings out great concern of exploring this organ and trying to understand this organ more closely and in more detail.

\subsubsection{Cardiac Chambers}

The heart consists of four chambers; two atria and two ventricle chambers[4]:

1. Right Atrium: receives oxygen-depleted blood and pumps it into the right ventricle.

2. Right Ventricle: pumps deoxygenized blood coming from the right atria to the lungs.

3. Left Atrium: receives blood from the lungs and pumps into the left ventricle.

4. Left Ventricle: responsible of pumping blood to all of the body.

\subsubsection{Cardiac Tissues}

Heart is composed of four layers:

1. Pericardium: the outer layer which encloses the heart

2. Epicardium: the outer tissue covering the heart; the epicardium is the outermost cell layer of the heart

3. Myocardium: the thickest layer; it contains the muscle cells

4. Endocardium: the innermost layer which has a smooth surface to allow blood flow.

The electrical potentials measured on the epicardium surface, namely the "epicardial potentials," are widely used in forward and inverse electrocardiography. These 
potentials are a representation of heart's electrical activity, and are used in this study as the cardiac sources.

\subsection{Electrical Activity of the Heart}

Here some terms that are used in describing heart's electrical activity will be explained.

\subsubsection{Action Potential Generation}

Each cell in our body is surrounded by a thin membrane. Different ions move across this membrane. Because ions are charged molecules, they cause an electric potential across two sides of a membrane, transforming each cell into a small battery. This imbalance is known as the membrane potential. Every resting cell in our body is slightly more negative inside then outside. Some cells that are called the excitable cells have the ability to reverse their membrane potential from negative resting values to slightly positive values. This rapid change in membrane potential is called an action potential (AP).

The heart muscle cells can generate action potentials. Each region of the heart has cells with slightly different action potentials. The Figure 2.1 shows a simplified shape of an action potential generated by the ventricular cardiomyocyte (muscle cell from the ventricle), the most common cell of the heart, Figure 2.2.

Generation of typical cardiac action potential after a stimulus can be explained with steps below:

1. The quick Na+ channels are opened which causes sodium to flow inward thus increasing the membrane potential through positive. Then depolarization occur around $+20 \mathrm{mV}$. 
2. Slower $\mathrm{K}+$ channels are opened and the outward flow of $\mathrm{K}+$ stops the rising potential due to $\mathrm{Na}+$.

3. $\mathrm{Na}+$ channels start closing while $\mathrm{K}+$ channels are still open.

4. Slow $\mathrm{Ca}++$ channels are opened and stay opened for approximately 20 millisecond which causes the plateau in membrane potential due to the inward $\mathrm{Ca}++$ flow.

5. $\mathrm{Ca}++$ channels are closed and repolarization occur with membrane voltage around $-90 \mathrm{mV}$.

The shape of cardiac action potential differs from the action potential of other excitable tissues in the body. The difference is the plateau present after depolarization in cardiac action potential. The main reason for this plateau is the 4 'th step explained above due to $\mathrm{Ca}++$ channels. This plateau does not occur in other excitable cells, such as nerve cells, because they do not have $\mathrm{Ca}++$ channels.

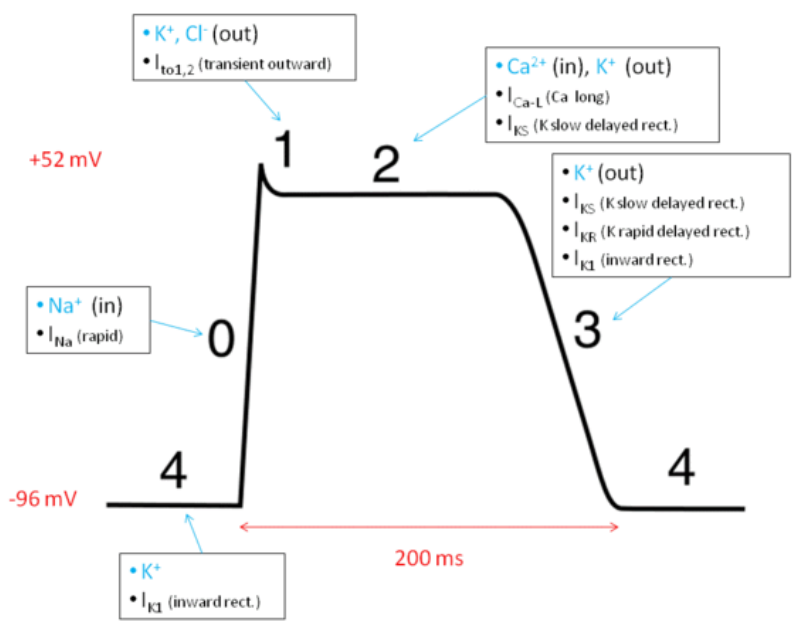

Figure 2.1: Action potential generated by a tissue in the heart [5] 


\subsubsection{Action potential propagation}

The action potential described in the previous section propagates, meaning that every activated cell stimulates its neighboring cells. Therefore, the generated action potential moves from one cell to another. The cardiac action potential differs significantly in different regions of the heart. Figure 2.2 illustrates different action potential shapes corresponding to different cells of the heart.

Although the action potential wave front changes for different tissues as seen in Figure 2.2 the generation of the typical cardiac action potential after the stimulus can be explained similarly, with steps introduces in the previous section.

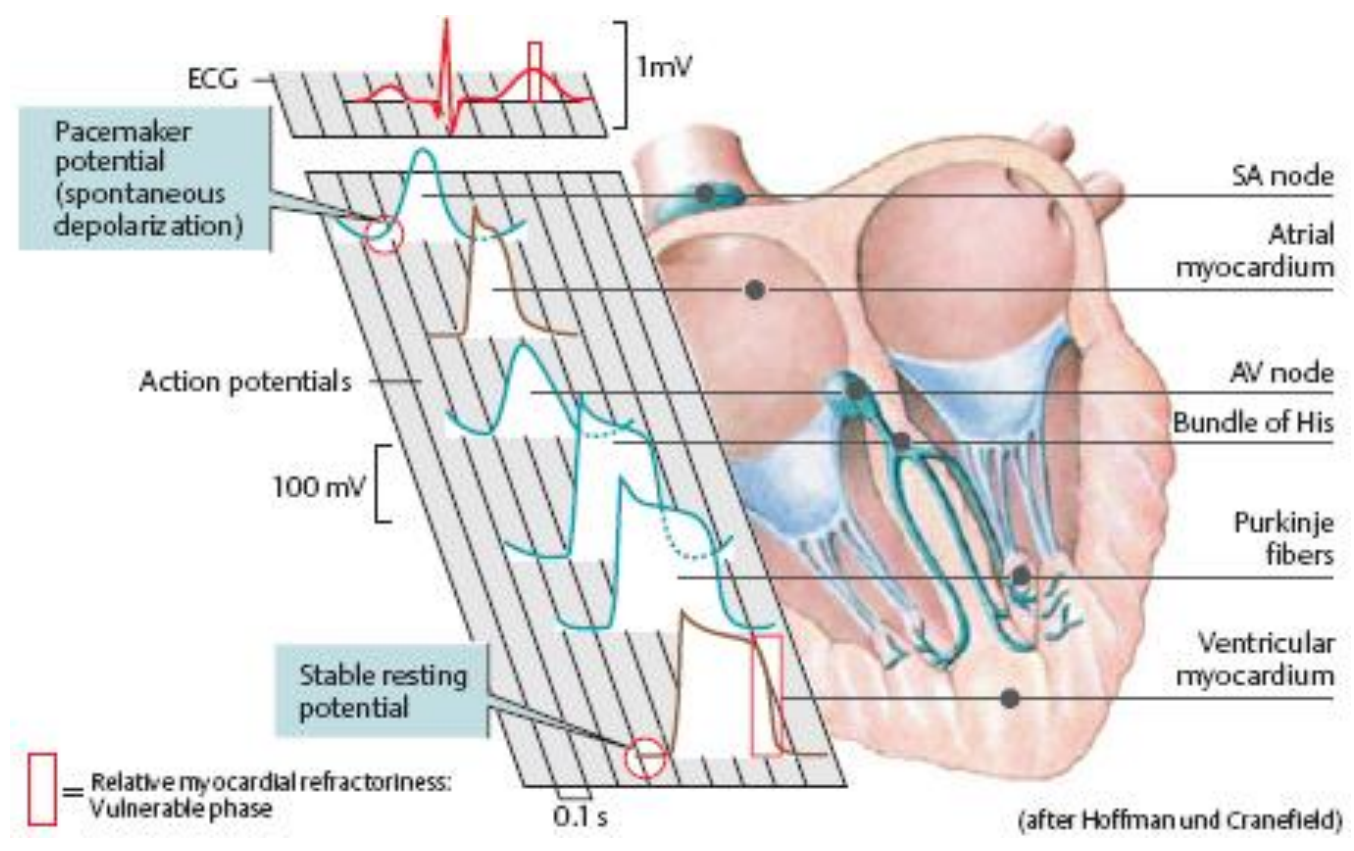

Figure 2.2: Action potential changes from one region to another [6] 


\subsubsection{Electrical Conduction System of the Heart}

Electrical activity of the heart starts by an electric stimulation in the so-called Sino Atrial (SA) Node. This stimulation point is the trigger point of a starting beat. Next, this electrical activity is transferred to the neighboring cells and an AP will be generated in each cell.

An electric wavefront moves all around the heart causing it to contract and pump blood to every single cell in the body. This contraction and the following relaxation is issued as an entire heart beat. The wavefront does not move uniformly on the heart because the conductivity in the heart is not the same in every direction (i.e., anisotropic conduction). The pumping effect is caused by this anisotropy property of the heart tissue.

The heart's electric activity is detected on the body surface by placing electrodes on the skin and measuring the electrical changes of the electric potentials with respect to a reference.

\subsection{Standard 12 Lead Electrocardiography (ECG)}

To measure the electric activity of the heart, ECG is used. The ECG works mostly by detecting tiny potential changes on the body surface when the heart muscle contracts during each heart beat. The term electrocardiogram was introduced by Willem Einthoven in 1893 [7]. Einthoven received the Nobel Prize for his life's work in developing the ECG. The ECG has evolved over the years; the standard 12-lead ECG is mostly used throughout the world [7]. In the 12-lead ECG, 12 waveforms are displayed. Figure 2.3. 


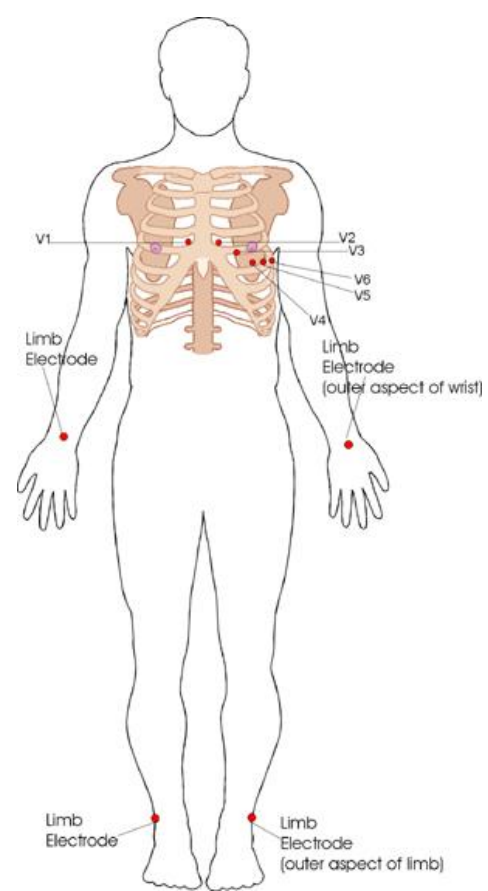

Figure 2.3: 12 lead Electrocardiograph electrode placement[8]

By looking at a typical electrocardiogram from lead II as shown Figure 2.4 , we can detect different wavefronts caused by the activity of different parts of the heart; this graph gives information of the functioning of heart chambers. Every section of this graph describes a certain activity of the heart; Figure 2.5 , displays the path of cardiac AP conduction, and the corresponding waves in the electrocardiogram with the conduction velocities, conduction times and self excitatory (intrinsic) frequencies [9]. 


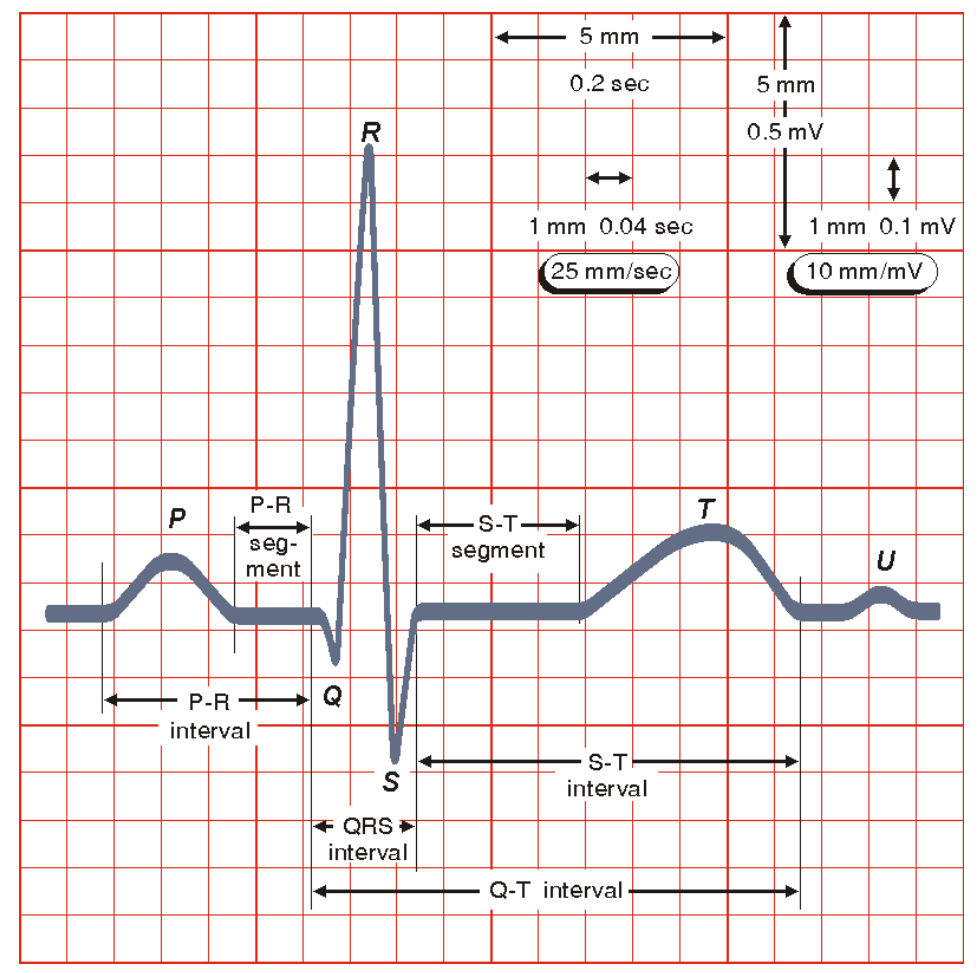

Figure 2.4: A single heart beat detected by Electrocardiograph [3]

The P-wave, the QRS wave and the T-wave are the important waves found in a typical ECG signal recorded from a normal heart:

P wave: $P$ wave is the depolarization of the atria that starts from the SA node which is the start point of the electrical activity of the heart. This depolarization moves all around the heart causing the atria to contract. This wave duration is around 80 100ms [9]. 


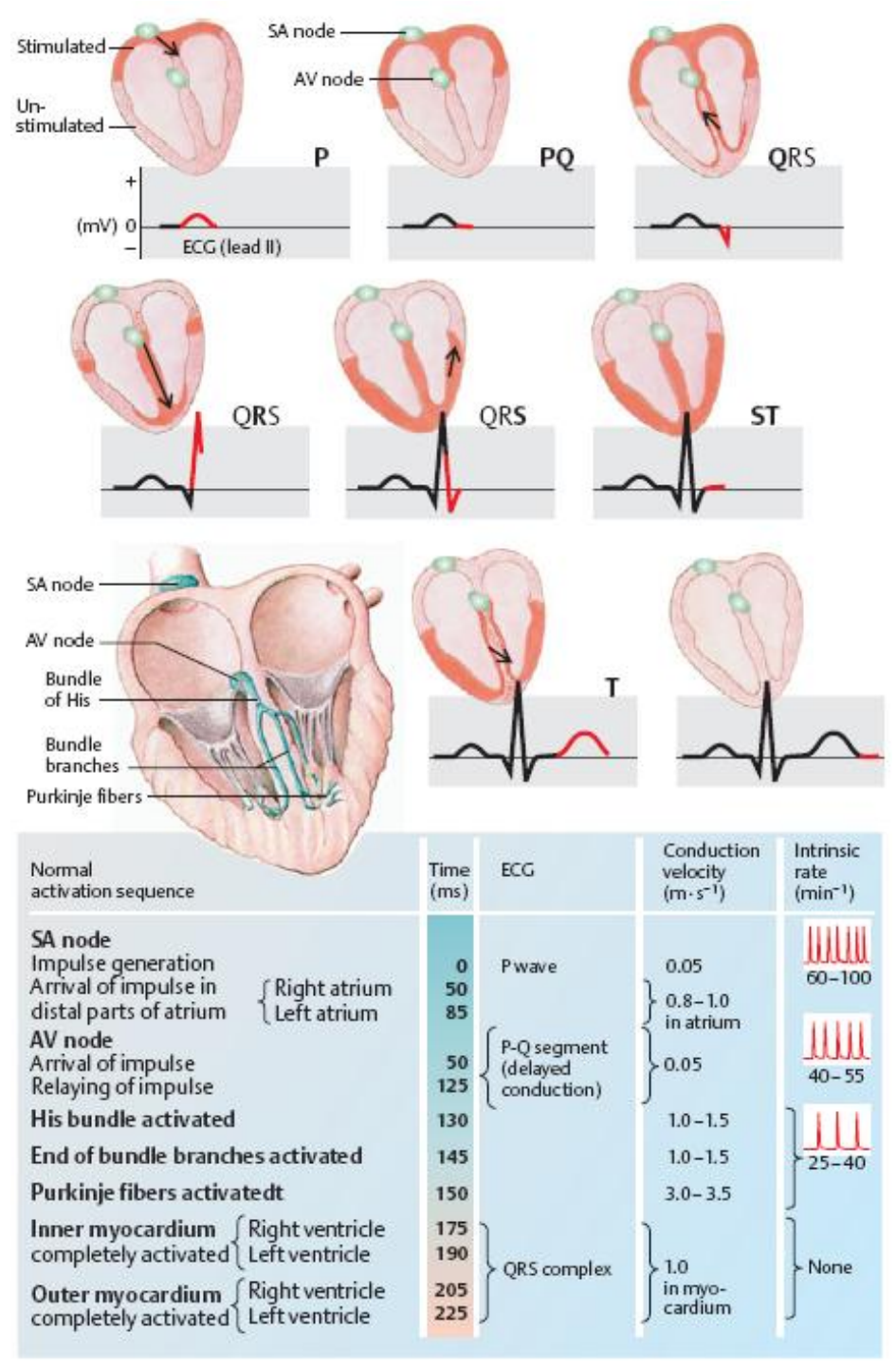

Figure 2.5: Cardiac AP conduction path and the corresponding wave on the ECG signal [9].

In Figure 2.5 the path of cardiac impulse conduction and the corresponding regions at the electrocardiogram with the conduction velocities, conduction times and self excitatory (intrinsic) frequencies [9].

QRS wave: The QRS wave or QRS complex is the result of ventricular depolarization and atrial repolarization. When the atria are completely depolarized, the wavefront is gathered in a node known as the AV node, and delayed for small time, and the AP is conducted through the Purkinje fibers to the ventricles and therefore causing them to start depolarizing. While the verticals start to contract, the 
atrial chambers start to relax; the corresponding electrical activity is detected on the body surface as QRS wave front.

T wave: The $\mathrm{T}$ wave represents the repolarization (or recovery) of the ventricles. The interval from the beginning of the QRS complex to the apex of the T wave is referred to as the absolute refractory period. The last half of the $\mathrm{T}$ wave is referred to as the relative refractory period (or the vulnerable period). Refractory period is the amount of time it takes for an excitable membrane to be ready for a second stimulus.

Any deformation of the ECG signal is translated as an abnormality in a certain activity of the heart. Thus clinicians can detect the abnormality of heart activity by simply looking at these graphs.

\subsubsection{Cardiac Arrhythmias}

Here we explain two most dangerous heart diseases which are caused by a malfunction in the cardiac chambers.

\subsubsection{Cardiac Re-entry Phenomenon}

In normal circumstances the excitation wave front follows a predetermined path because all other paths are obstructed with tissues in refractory period. But sometimes due to certain problems the wave front can find other ways to spread and after a period it comes back and simulates the same tissues again and again unless the conduction is resynchronized with electroshock [4]. 


\subsubsection{Ventricular Fibrillation}

Ventricular fibrillation is the most serious cardiac arrhythmia and it can cause death if not treated within 1 to 3 minutes. The main reason for this arrhythmia is Re-entry Phenomenon which distorts the synchrony of the heart muscles. Due to this asynchrony sufficient Blood cannot be pumped to body which results in unconsciousness in a few seconds and death in a few minutes [4].

\subsection{Forward Problem of Electrocardiography}

Obtaining the body surface potentials corresponding to potentials on the Epicardial layer of the heart is one of the major problems in biomedical engineering, which is called the Forward Problem of Electrocardiography. This problem has several applications:

- Simulation of ECG with computer heart models

- Solution of reciprocal problem of determining currents over the heart due to the current sources on body surface

In the forward ECG problem, a transfer function is obtained which relates the potentials on the epicardium surface to the potentials on the torso surface; this transfer function could later be used to find the solution to the inverse problem.

\subsubsection{Geometric Information}

Before solving the forward ECG problem, geometric information about the cardiac and surrounding tissues should be obtained. Where medical imaging methods like Magnetic Resonance Imaging (MRI) and Computed Tomography (CT) have been used to gather this information [2]. 


\subsubsection{Numeric Methods for Solving the Forward Problem}

There are a number of numerical approaches for solving this problem in the literature;

Boundary element method (BEM): In this method, we assume all torso regions are isotropic, thus only the boundaries of regions are taken into account. This method is seen as a surface method.

Finite Element Method (FEM): The main idea of this method is to divide the structure into small volumes and describe the behavior of each element by taking into account their physical and biological properties. In FEM, a distributed physical system to be analyzed is divided into a number of discrete elements. The complete system may be complex and irregularly shaped, but the individual elements are easy to analyze. The division into elements may partly correspond to natural subdivisions of the structure [10] [11].

Finite Difference Method (FDM): Finite-difference method approximates the solutions to differential equations by replacing derivative expressions with approximately equivalent difference quotients. In our problem we first generate a grid, where we want to find an approximate solution, then approximate the derivatives in the partial differential equation by linear combinations of function values at the node points.

Finite Volume Method (FVM): Similar to the finite difference method, values are calculated at discrete places on a meshed geometry. In this method, volume integrals in a partial differential equation are converted to surface integrals, using the divergence theorem.

In the literature, FEM and BEM approaches are compared [10] [11]. In BEM, only the boundaries of the tissues with different conductivity values are discretized [12] Thus, it is obvious that implementation of BEM is less complex then FEM, which requires the discretization of the entire volume. Adaptive methods can be used to increase the performance of both of these methods [12] [13]. 


\subsection{Inverse Problem of Electrocardiography}

In this section, first a brief introduction to the inverse problem for ECG will be given. Next, a literature survey on the algorithms that have been applied will be presented.

\subsubsection{Introduction to Inverse Problem of Electrocardiography}

The inverse problem of electrocardiography is the problem of finding the potential distribution over the cardiac surface using the potentials that are measured on the body surface. Inverse problem of ECG opens doors for non invasive detection of epicardial potentials, and new cardiac imaging modalities. This solution would give the clinicians the ability to detect and diagnose cardiac abnormalities or the opportunity to monitor the effects of drugs on cardiac activity. This shows the advantages of what an optimum solution for this problem would provide for the clinicians. By simply presenting the advantages of the solution of this problem, we could immediately understand the importance of any improvement or unique method in solving this problem.

One most common ground with different inverse problems are that they are concerned with determining causes for a desired or an observed effect or calibrating the parameters of a mathematical model to reproduce observations. Inverse problems most often are not well-posed; they might not have a solution in the strict sense, solutions might not be unique and/or might not depend continuously on the data.

When a problem is said to be well posed, two properties should be satisfied:

1) The solution should be unique.

2) The solution should only depend on the data.

One of the main difficulties about inverse electrocardiography problem is the nonuniqueness of the problem. Different cardiac source distributions may result in the 
same body surface potentials, which may result in physiologically meaningless solutions. In order to overcome this problem, physiological constraints can be used. If the solution of the problem depends not only on the data but also on the noise, the problem becomes ill posed, which is the case in the inverse problem of ECG; even a small amount of noise in the data would make the solution unstable and the solution would be unreliable.

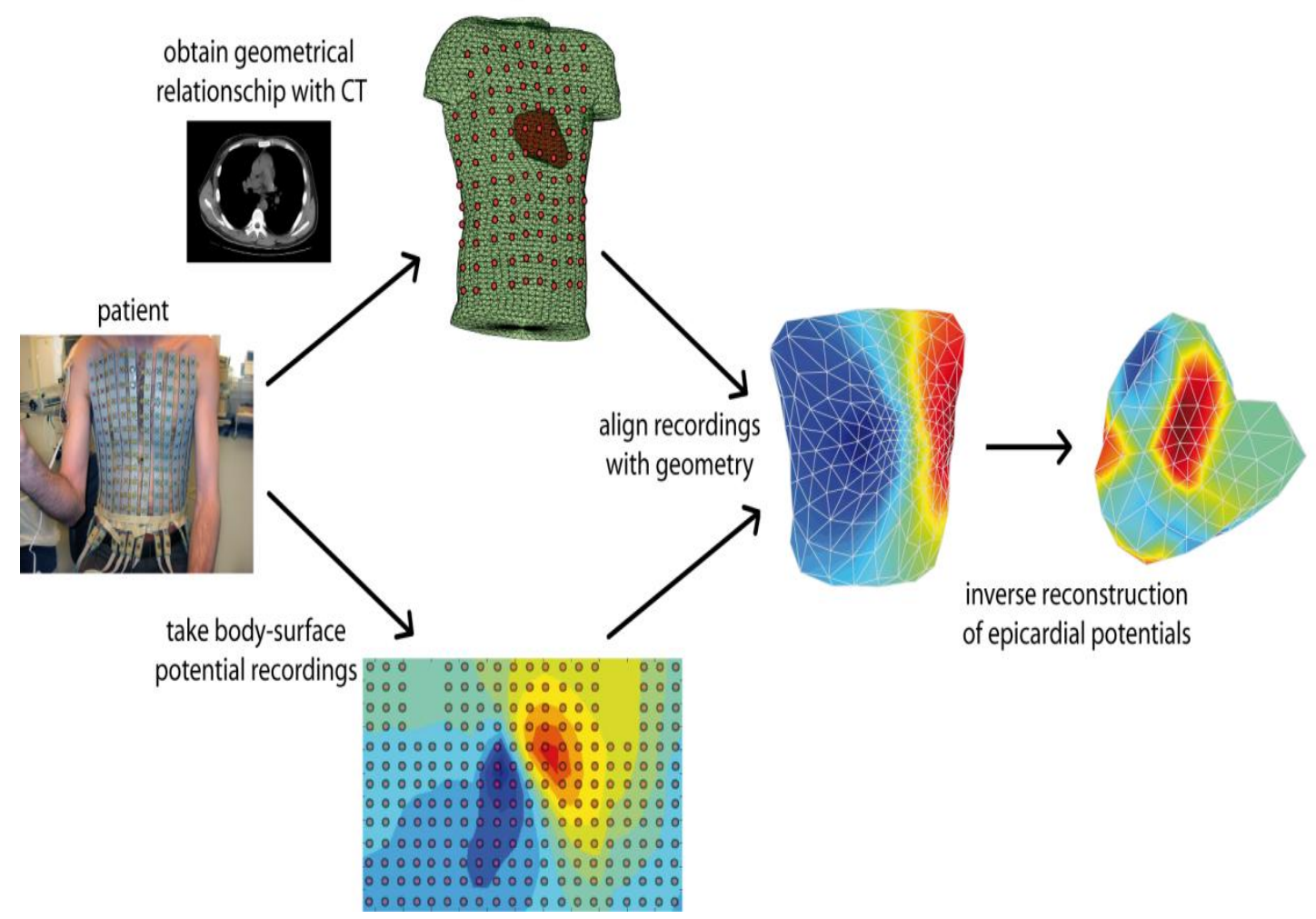

Figure 2.6: Inverse ECG [14].Using body surface potentials and geometric information to calculate epicardial potentials 


\subsubsection{Methods in the Literature to Solve the Inverse ECG Problem}

Throughout the years, inverse problem of ECG has been of interest to engineers and a number of solutions have been presented for this problem. But before solving the problem, a model for the cardiac electrical activity should be considered. In previous works, this has been done in different ways. Some try to model the heart by one dipole which is located in the middle of the heart [15]. Other methods use more than one dipole [16] [10].

The approaches for solving the inverse ECG problem can be categorized into two main categories:

1. Spatial approaches

2. Spatial temporal approaches

In each of these methods, different properties of the problem are taken into account; some only take spatial properties of the problem, others concentrate on temporal properties or both.

\subsubsection{Spatial Approaches}

These methods only use spatial constraints to solve this ill posed problem and the temporal behavior of the problem is not taken into account.

\section{a. Tikhonov Regularization}

This regularization method is one of the most common regularization methods used for solving ill posed problems. It is regarded as adding spatial constraints to the 
typical least square minimization problem [17]. This method will be explained in detail the following section 3.2.

\section{b. Truncated Singular Value Decomposition (TSVD)}

TSVD is another method that is widely used in regularization problems. TSVD is a type of Singular Value Decomposition (SVD); the difference is that in TSVD, one calculates the decomposition approximately by including only some of the singular values in the reconstruction. The main motivation in TSVD is the elimination of the small singular values of the transfer matrix to overcome the ill-posedness [18]. By eliminating the small singular values the system would become regularized, but this would also erase the higher frequency components of the solution, hence the system suffers from over smoothing.

\section{c. Bayesian Maximum a Posteriori (MAP) Estimation}

In this approach, the aim is to maximize the conditional a posteriori Probability Density Function (PDF) of the epicardial potentials given the BSP's and an a priori PDF of epicardial potentials. This solution usually takes into account only the spatial properties because in the solution usually each time instant is taken separately from each other. This algorithm could be extended to spatio-temporal solutions [18] [ 19]. 


\subsubsection{Spatial Temporal Approaches}

In this section spatio-temporal methods are given.

\section{a. Multiple Constraints}

The inverse problem of ECG has temporal properties as much as spatial; solving the problem without considering the temporal relations in the problem would mean loss of information. Thus methods that have been applied to solve the problem spatially have also been adapted to include the temporal information in addition to the spatial information. As mentioned above, Tikhonov regularization method is the most common regularization method; and the additional spatial and temporal constraints can be included in the cost function to benefit from all the available information [20].

\section{b. State-Space Models}

State space models like Kalman Filters are methods that benefit from the spatialtemporal behavior of the epicardial potentials. The main difficulty of using Kalman filter for electrocardiographic imaging is the determination of the State Transition Matrix (STM). This matrix is critical because it determines the spatio-temporal relationship of epicardial potentials for two consecutive time instants. Some methods use the identity matrix as the STM matrix [21] while other studies calculate State Transfer Matrix (STM) using true epicardial potentials or training sets [22] [23] [24] [25].

\section{c. Hybrid Methods}

There are a number of other approaches that use combinations of algorithms to find a solution to the inverse ECG problem. For example, one such study combines the 
least squares QR with TSVD, another combines genetic algorithms and Laplacian weighted minimum norm [26] [27] [28], etc.

\subsubsection{Using Genetic Algorithm in Inverse ECG Problem}

The regularization methods reconstruct epicardial potentials from body surface potentials by smoothing or truncating the noise. Studies in the literature have used Genetic Algorithm (GA), which is a stochastic search technique, in combination with various regularization methods to solve the inverse ECG problem [29] [30]. Where in these methods a solution of a regularization method (i.e. Least square) is treated as an initial population for the genetic algorithm. the results show that by combing the regularization methods with genetic algorithms (GA), the performance can be improved [30].

\subsubsection{Other Approaches to Obtain Cardiac Electrical Activity}

Methods like Laplacian weighted minimum norm (LWMN) algorithm is used to determine the current dipoles at myocardium [28]. They found the location of the arrhythmias in three dimensional myocardium, which distinguishes this study from others that try to find the location of arrhythmia with solutions of epicardial or endocardial potentials. Due to spatial smoothing effects of Laplacian operator, they also applied a recursive weighting algorithm to detect even small arrhythmia sites. Farina et al. suggested using the information obtained from the simulations as a priori information for inverse ECG solution [31]. For that purpose they used cellular automaton to calculate the transmembrane potentials for the patient's anatomical data obtained by MRI. Then using FEM, the Epicardial potentials are calculated from those transmembrane potentials. They compared three regularization methods which are zero'th order Tikhonov, Twomey and a stochastic regularization method. 
The a priori information used for Twomey and stochastic regularization are obtained from those calculated epicardial potentials and only spatial regularization is used. The results show that the best solutions are obtained with stochastic regularization, but its computational cost is higher than others. Also Twomey regularization helps to avoid over-smoothing which is a typical problem for the zero'th order Tikhonov regularization. In another study the same group determines the slope of transmembrane potential and action potential propagation velocity using body surface potentials [32]. The importance of this study is that they optimize the excitation velocity for different tissues in the patient specific cellular automaton model such as bundle branches and Tawara nodes. By this way a model of the patient's heart is constructed where the effects of therapy might be simulated, and the infracted tissues could be determined.

Another study which tries to fit a model according to the measured BSP's is studied by $\mathrm{He}$ et al. for 3-D myocardium model [33]. In their study, an anisotropic heart model is used to obtain more realistic results with different excitation velocities for longitudinal and transverse fiber direction and 82 different myocardial segments which results in 82 current dipoles are assumed. They also used cellular automaton model to simulate the BSP's. They first obtain the anatomical information with CT and use this data to build a thorax model. Then they optimize the parameters in their model with a comparison of measured and simulated BSP's. After that, using those optimized parameters, they determine the myocardial activation times. They have also published a similar study where they determine the transmembrane potentials [33].

The work by Nash et al. is important in the sense that it is an in vivo study that measures BSP's and epicardial potentials simultaneously [34]. Earlier studies use in vitro experiments with a dog heart placed in a torso tank [35] [36] or does not have the needed resolution because of the technological limitations [37] [38]. In order to measure epicardial and body surface potentials simultaneously, they implemented a sock electrode to the ventricles of a pig heart and the wire for epicardial measurements are exited near the diaphragm. Also a suture snare is inserted to the left anterior descending (LAD) coronary artery to occlude it whenever necessary during the experiment, and then they closed the chest. 
They have stated that with that experiment they were able to observe the slow conduction velocity at the ischemic region and measure the epicardial and BSP's simultaneously. As the authors stated, this study could serve well to validate the inverse electrocardiography solutions. Ramanthan et al. tested the technique they called electrocardiographic imaging which is same as inverse problem of electrocardiography for human subjects [39]. In their study they tested their imaging system for normal sinus rhythm, right bundle branch blocks, right and left ventricular pacing and atrial flutter. The first step in their system is the measurement of body surface potentials from 224 points with a vest electrode. Then they used CT with an axial resolution of 0.6 to $1 \mathrm{~mm}$ to obtain epicardial surface geometry and the positions of the vest electrodes.

In the signal processing part, first the preprocessing module acquires ECG signals with noise filtration, baseline correction etc, at the second part the segmentation process is carried on and the CT images are ready for calculating transfer matrix with BEM at the third step. Finally at the fourth step Tikhonov regularization and generalized minimum residual error algorithms are applied to the data to overcome ill-posedness of ECG signals. They obtained epicardial potential maps, epicardial electrograms, activation time isochrones maps and recovery times. Rudy et. al. also obtained the patent for that system in 2006. 


\section{CHAPTER3}

\section{METHODS}

As explained before, regularization of inverse problems involves introducing additional information on the solution in order to solve the ill-posed problems. This information is usually in terms of restrictions for smoothness or bounds on the vector space norm. From a Bayesian perspective this would correspond to imposing certain prior distributions on model parameters.

Informally, regularization is defined as stabilizing an ill posed problem in a manner that yields approximate solutions, often by incorporating prior information.

In this chapter, we start by introducing the problem in hand and the solutions introduced in the literature. We then continue by the proposed method in this study.

\subsection{Problem Definition}

In the forward problem of ECG, the relation between the torso and the epicardial potentials are linear, and additive noise that comes from measurement perturbations and inhomogeneities in the model corrupts the measurements:

$\mathbf{Y}=\mathbf{A X}+\mathbf{N}$

Here, $\mathbf{Y} \in R^{M \times T}$ and $\mathbf{X} \in R^{P \times T}$ are matrices representing the torso potentials and the epicardial potential respectively, where $M$ is the number of leads on the body 
surface, $\mathrm{P}$ is the number of leads on the epicardial surface, and $\mathrm{T}$ is the number of time instances.

$\mathbf{A} \in R^{M \times P} \quad$ represents the transfer matrix, which is obtained by the solution of forward problem of ECG. $\mathrm{N}$ is the measurement noise of the same size as $\mathbf{Y}$.

Although the notation that we are going to use equation 3.1 in this section is in the matrix form but there are two other states that this problem has been dealt with.

Equation (3.1) can be stated in vector notation

$$
\mathrm{y}(\mathrm{k})=\mathbf{A x}(\mathrm{k})+\mathrm{n}(\mathrm{k}) \quad k=1,2,3 \ldots T
$$

Where $\mathrm{k}$ resembles each time instant.

the third notation which equation 3.1 is stated is the augmented case.

$$
\left[\begin{array}{c}
y(1) \\
y(2) \\
\vdots \\
\vdots \\
y(T)
\end{array}\right]=\left[\begin{array}{ccc}
A & 0 & 0 \\
0 & A & 0 \\
0 & 0 & A
\end{array}\right]\left[\begin{array}{c}
x(1) \\
x(2) \\
\vdots \\
\vdots \\
x(T)
\end{array}\right]+N \quad \quad \bar{y}=\overline{\mathrm{A}} \overline{\mathrm{x}}+\mathrm{n}\left(0, \mathrm{C}_{\mathrm{n}}\right)
$$

We proceed by equation 3.1 when problem arises when one tries to find the inverse of $\mathbf{A}$ to calculate $\mathbf{X}$ because $\mathbf{A}$ is not square matrix therefore to calculate this calculated equation 3.4 .

$$
\mathbf{A}^{-1}=\left(\mathbf{A}^{\mathrm{T}} \mathbf{A}\right)^{-1} \mathbf{A}^{\mathrm{T}}
$$

Figure 3.1.1 shows the Eigen values of $\mathbf{A}^{\mathbf{T}} \mathbf{A}$ from largest to smallest Most of these eigen values are close to zero therefore The condition number of $\mathrm{A}$ which is calculated by dividing the largest eigen value of $\mathbf{A}$ to the smallest eigen value is $10^{19}$ which shows that this matrix is very ill-conditioned. Thus $\mathbf{A}^{\mathbf{T}} \mathbf{A}$ matrix is near singular and finding its inverse is not possible. 


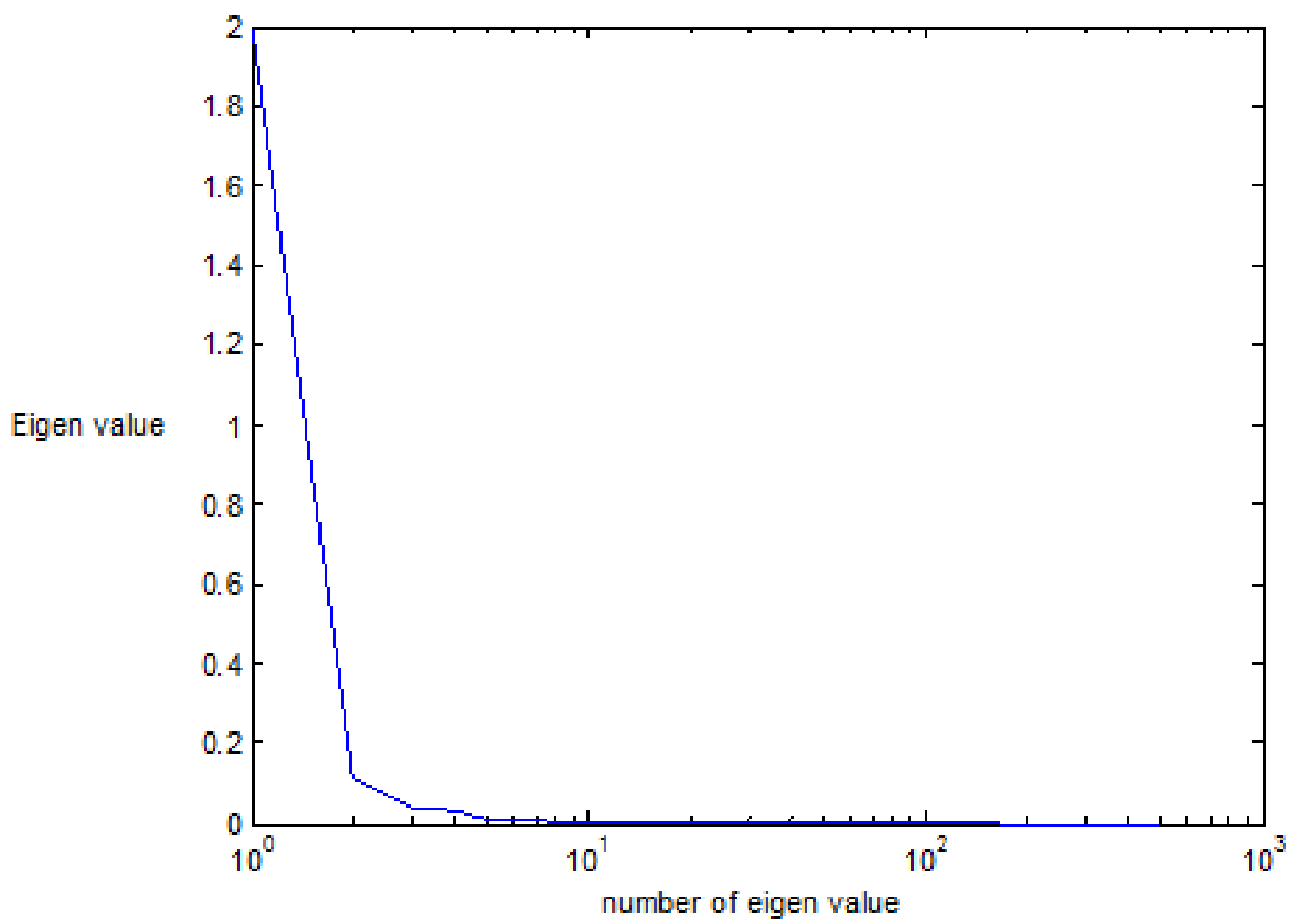

Figure 3.1.1: Eigen values of transfer matrix $\mathrm{A}^{\mathrm{T}} \mathrm{A}$.

Due to this ill-posedness, any small noise causes large perturbation in the inverse solution making it unreliable and often unmeaningful. Different regularization methods have been used in literature to solve this problem. One of the most famous regularization methods is Tikhonov regularization. In the rest of this chapter, we present some of the regularization methods that we used in our thesis study.

\subsection{Tikhonov Regularization}

In this approach the solution to equation 3.1 is

$\hat{\mathrm{x}}_{\lambda}=\operatorname{argmin}_{\mathrm{x}}\left(\left(\|\mathrm{Ax}-\mathrm{y}\|^{2}+\lambda^{2}\|\mathrm{Rx}\|^{2}\right) \quad\right)$

Where $y \in R^{M \times 1}$ denotes torso potentials for one time instant and $x \in R^{P \times 1}$ represents epicardial potentials for one time instant.

Which can also be expressed as: 
$\hat{x}_{\lambda}=\left(A^{T} A+\lambda^{2} R^{T} R\right)^{-1} A^{T} y$

As one can see from equations (3.5) and (3.6), Tikhonov solution can be separated into two parts; the first part is the solution of the least square method, and the second part is a constraint added to the overall cost function to stabilize the solution.

There are different methods used in the literature to calculate an optimum $\lambda$ such as Composite Residual and Smoothing Operator (CRESO) [40], Generalized CrossValidation (GCV) or maximum likelihood estimator [41][42], and the L-curve approach, which is the most popular method to obtain $\lambda$ [43][44]. The mathematics behind the L-curve approach is not discussed here, but to explain briefly, the residual norm, $\|A x-y\|$, is plotted against the solution norm, $\|\mathrm{Rx}\|$, corresponding to a range of regularization parameters, in log-log scale. In this method there is a tradeoff between the "size" of the constraint norm $\|\mathrm{Rx}\|$ and the quality of the fit that it provides to the given data $\|A x-y\|$. Thus calculating the regularization parameter is very important. Note that if too much regularization, or damping, is imposed on the solution, then it will not fit the given data $\boldsymbol{Y}$ properly and the residual $\|\mathrm{Ax}-\mathrm{y}\|$ will be too large. On the other hand, if too little regularization is imposed then the fit will be good but the solution will be dominated by the contributions from the data errors, and hence $\|\mathrm{Rx}\|$ will be too large. By selecting an appropriate regularization parameter the solution to this problem could be found.

The L-curve plot has an L-shaped form, and the regularization parameter corresponding to the corner of this L-shape is taken as the optimum value for the regularization parameter. Figure 3.1.2 shows an example of the L-curve [14]. 


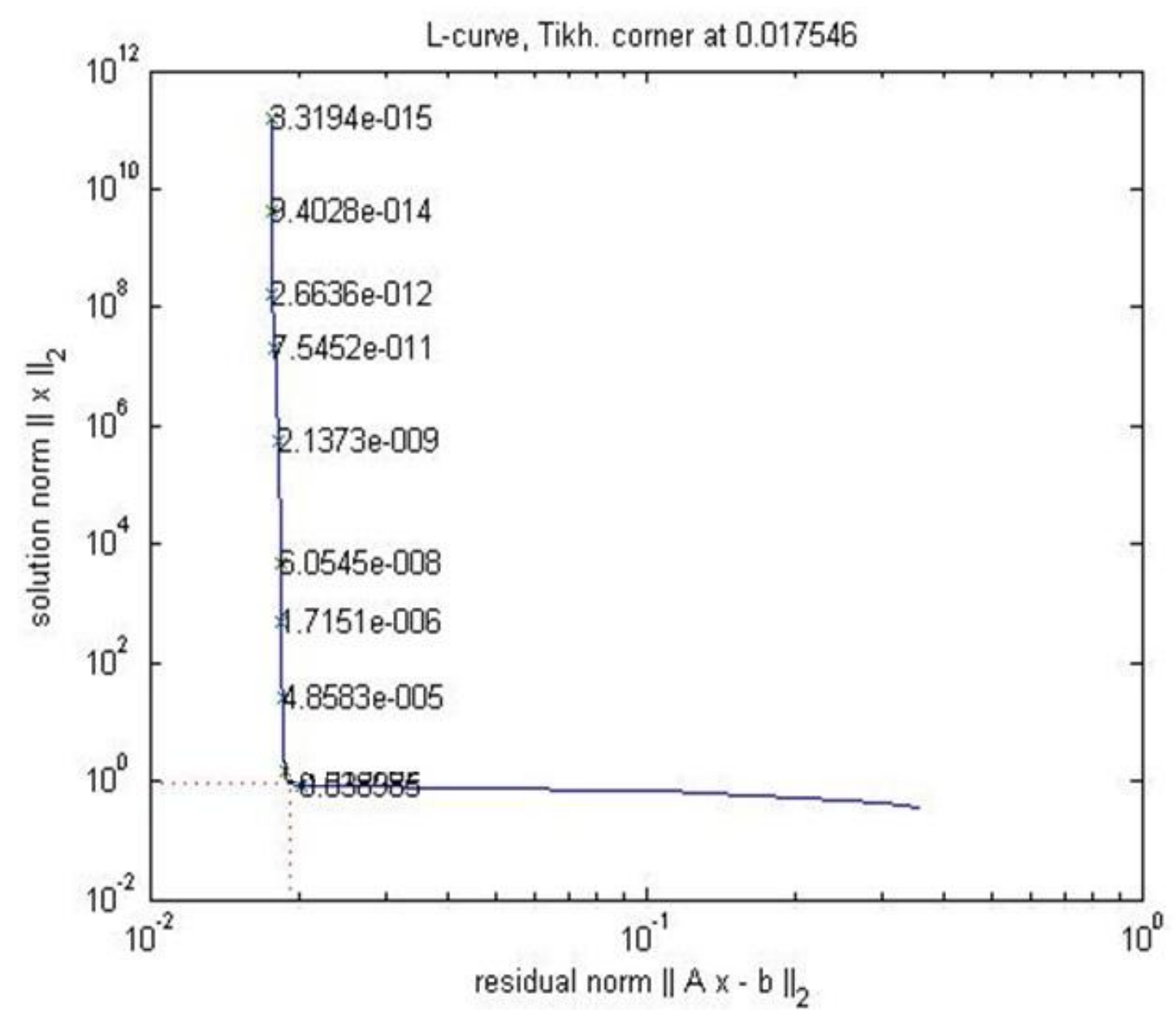

Figure 3.1.2: L-curve

To demonstrate the importance of correct choice of the regularization parameter, a simple example is given here. In this example, we have a source distribution consisting of a curve with two Gaussian-shaped peaks, which moves from left to right [45].

The spatial curve for the first time instant is defined as

$$
x(n)=\frac{1}{2 \sqrt{2 \pi}} \exp \left(-\frac{\left(n-m_{1}\right)^{2}}{2 \sigma_{1}^{2}}\right)+\frac{1}{4 \sqrt{2 \pi}} \exp \left(-\frac{\left(n-m_{2}\right)}{2 \sigma_{2}^{2}}\right)
$$

Where $m_{1}$ and $\sigma_{1}$ and $m_{2}$ and $\sigma_{2}$ are constants, and $n$ is the time index. The forward matrix used to obtain the measurements is given by the relation: 


$$
A(i, j)=\frac{1}{N \sqrt{2 \pi} \sigma} \exp \left(-\frac{\left(\frac{(i-j)^{2}}{N}\right)}{2 \sigma^{2}}\right)
$$

We solve this inverse problem by applying Tikhonov regularization. First the optimum regularization parameters are obtained for each time instant using the Lcurve approach, then these optimum parameters are increased by 0.2 units to observe the effects of a non-optimal regularization parameter. The original source distribution and the resulting optimal and non-optimal inverse solutions are plotted in Figure 3.1.3. This example shows that by changing the regularization parameter even slightly, the reconstructed image is deformed. By looking at the distributions in Figure 3.1.3, the change in the solution corresponding to a change in the regularization parameter is obvious. This change could be quantified using metrics such as the correlation coefficient, relative error, etc. 


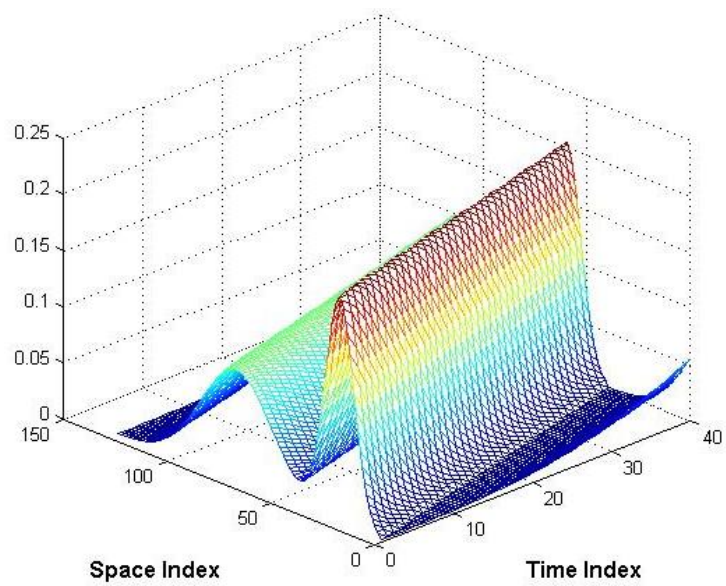

a)

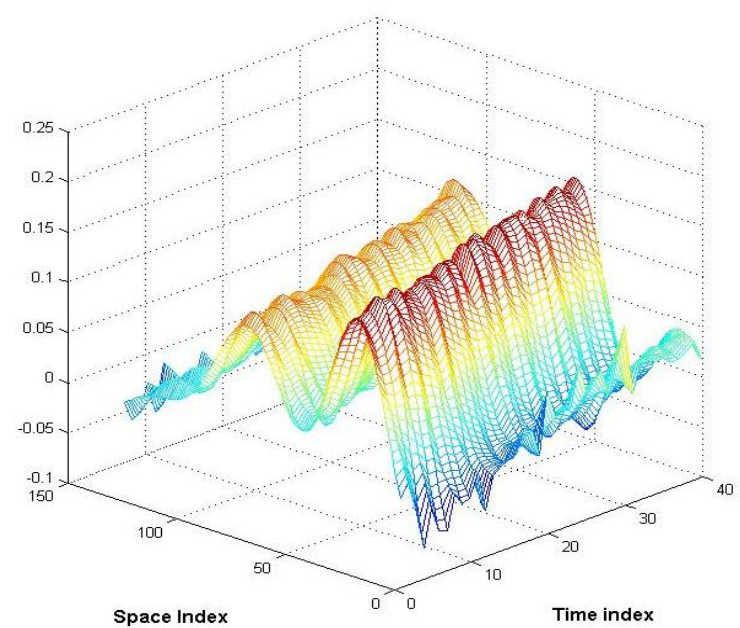

b)

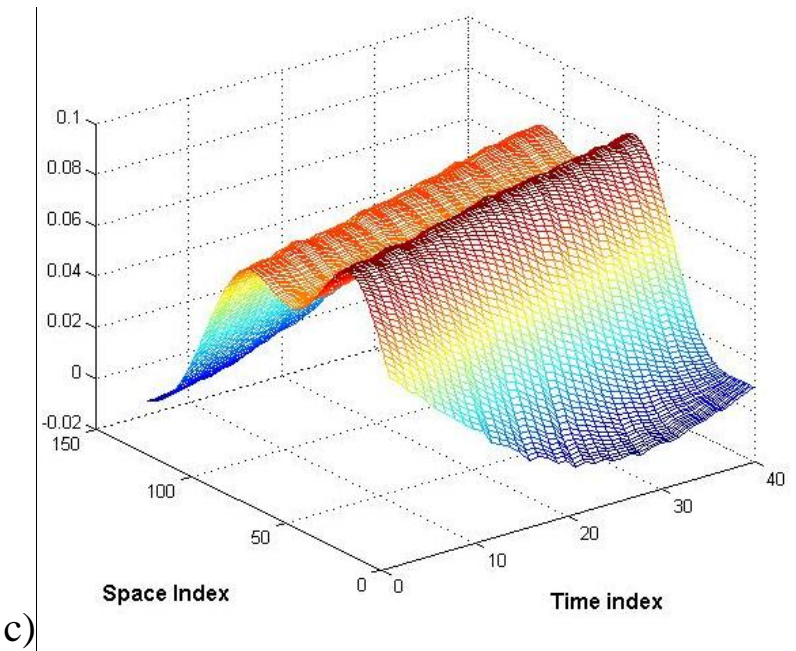

Figure 3.1.3: a) Two Gaussian-shaped peaks (original image), b) Tikhonov solution of the problem. c) Tikhonov solution with a slight change in the regularization parameter 


\subsection{Multiple Constraints Method}

One drawback to using constraints to stabilize the inverse solutions is that each constraint introduces a bias on the solution. Furthermore, regularized solutions are quite sensitive to the value of the regularization parameter. One way to reduce the effects of bias introduced by the constraints is to combine several constraints rather than using only one. Some studies in this field that combine multiple constraints are the admissible solution approach [46] and multiple constraints approach that adds each constraint to the cost function via corresponding regularization parameters [47]. It is the second approach that we considered in our studies. This approach could be studied under two sub-categories: 1) multiple spatial constraints, 2) multiple spatial and temporal constraints. In the next two sections, these approaches will be explained.

\subsubsection{Multiple Spatial Constraints}

In this approach, we extend the cost function in equation 3.5 to use multiple spatial constraints instead of using just one spatial constraint. The new optimization problem for $\mathrm{n}$ constraints with $\mathrm{n}$ regularization parameters can be given as:

$$
\min _{\mathrm{x}}\left(\|A X-Y\|^{2}+\sum_{\mathrm{i}=1}^{\mathrm{n}} \lambda_{\mathrm{i}}^{2}\left\|\mathrm{R}_{\mathrm{i}} \mathrm{X}\right\|^{2}\right)
$$

The solution to equations 3.9 is

$\widehat{X}=\left(A^{T} A+\sum_{i=1}^{n} \lambda_{i}^{2} R_{i}^{T} R_{i}\right)^{-1} A^{T} Y$

As it is obvious from the formulation above, there is no restriction on how many constraints that could be used to solve the inverse problem. The challenge is to find optimum values for $\lambda_{\mathrm{i}}$. more explanation on this approach is given in Appendix A. 


\subsubsection{Multiple Spatial and Temporal Constraints}

As mentioned before the epicardial potentials are spatially and temporally correlated, thus adding a temporal constraint to the solution is essential. In this section, a new cost function is introduced by adding temporal constraints to the expression in Equation 3.9.

$\min _{\mathrm{x}}\left(\|\mathrm{Ax}-\mathrm{Y}\|^{2}+\sum_{\mathrm{i}=1}^{\mathrm{k}_{\mathrm{s}}} \lambda_{\mathrm{i}}\left\|\mathrm{R}_{\mathrm{i}} \mathrm{x}\right\|^{2}+\sum_{\mathrm{j}=1}^{\mathrm{k}_{\mathrm{t}}} \eta_{\mathrm{j}}\left\|\Gamma_{\mathrm{j}} \mathrm{x}\right\|^{2}\right)$

Where $\Gamma_{j}$ and $\mathrm{R}_{\mathrm{i}}$ are the temporal and the spatial regularization matrices.

To calculate a solution to equations (3.11) we used pre-digonalaization method [20]. More detailed explanations are given in Appendix B.

Usually, the sizes of the $\mathrm{R}$ and Gamma Matrices are not the same, therefore we cannot use the compact form of the solution introduced in Equation 3.11.

The solution to the augmented problem can be given as:

$\hat{\bar{x}}_{\lambda_{i} \eta_{j}}=\left(\bar{A}^{T} \bar{A}+\sum_{i=1}^{n} \lambda_{i}^{2} \bar{R}_{i}^{T} \bar{R}_{i}+\sum_{j=1}^{k_{t}} \eta^{2}{ }_{j} \bar{\Gamma}^{T}{ }_{j} \bar{\Gamma}_{j}\right)^{-1} \bar{A}^{T} \bar{y}$

Where $\bar{x}, \bar{y}$ and $\bar{A}$ are as explained in equation 3.3 and $\overline{\mathrm{R}}=\mathrm{I}_{\mathrm{T}} \otimes \mathrm{R}$ and

$$
\bar{\Gamma}=\Gamma \otimes \mathrm{I}_{\mathrm{P}}
$$

In both types of multiple constraints scenarios, it is still a problem to find optimum regularization parameters. For the case where only two constraints are used (one spatial and one temporal constraint, or two spatial constraints), one can use the sacalled "L-surface," in which the residual norm and the two constraint norms are plotted in log-log scale as a function of two regularization parameters, $\lambda \lambda$ and $\eta$ [20]. An example of an L-surface is given in Figure 3.1.4. 


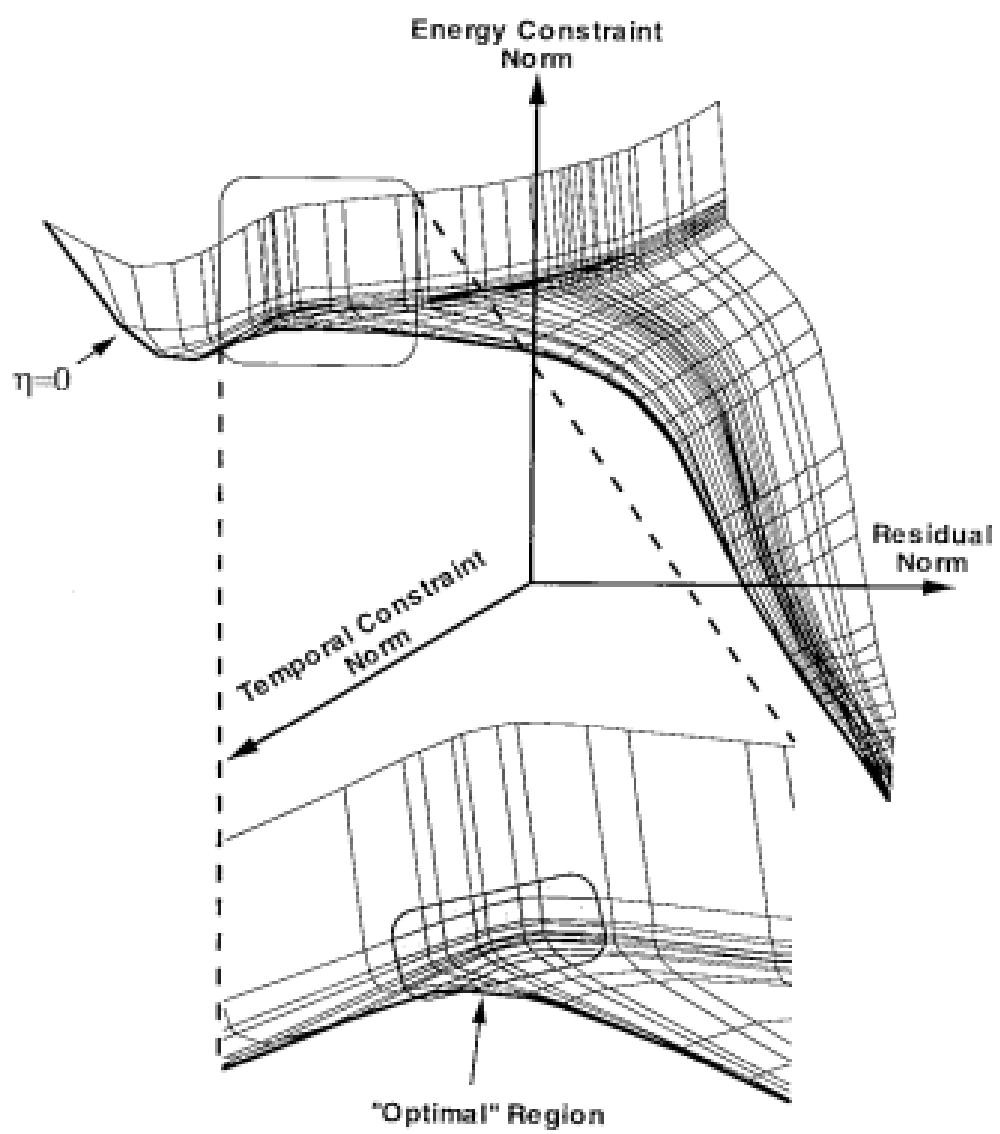

Figure 3.1.4: L-surface

Showing the $\log$ of the two-norms of the two constraint terms as a function of the $\log$ of the residual norm. an enlargement of the "corner" where optimum regularization parameters are localized are given in the lower figure. The upper figure illustrates the surface over a wide variety of regularization parameters [20].

This method is an extension to the L-curve method. Similar to the L-curve method, the corner of the L-surface corresponds to an optimum $(\lambda, \eta)$ pair.

This method not only is difficult to implement, but also very difficult to use for more than two parameters. Calculating the parameters at the corner of this L-shaped surface is time consuming and difficult. Therefore in this study we used Genetic Algorithms to automatically find the optimum regularization parameters. This new 
approach can find two regularization parameters and be extended to include more than two regularization parameters.

In the following sections, we explain two methods to obtain multiple regularization parameters.

\subsection{Regularization parameter calculation}

To determine the regularization parameters we used two different methods:

- Determining the regularization parameters using real data,

- Determining the regularization parameters using genetic algorithms (GA).

These approaches are discussed in the next two sections.

\subsubsection{Regularization Parameter Calculation Using Real Data (Maximum CC method)}

In this approach, we use the real epicardial potentials to detect the regularization parameters that produce good solutions to the inverse problem. The regularization parameters are selected so that maximum correlation coefficient (MCC) values could be achieved by the solution. This approach is not appropriate in clinical use, since it requires comparison of every solution with the real data to calculate the $\mathrm{CC}$ value and to make the optimum parameter selection. However, it provides a base-line to compare the GA-based regularization parameter selection algorithm results.

In the first step of this algorithm, we determined the number of regularization parameters that are going to be used. Next, a confidence region was selected for each regularization parameter (i.e., maximum and minimum values of regularization parameters to be tested). While changing one of the regularization parameters step by step in the entire confidence region, the other parameters are kept constant, and solutions along with associated CC values are obtained for all regularization 
parameter combinations using the formulation discussed in sections (3.3.1) or (3.3.2). The regularization parameter combination (pair, if there are only two constraints) that yields maximum value of the $\mathrm{CC}$ value is picked as the "optimum" regularization parameter set to calculate the solution. This algorithm is summarized in Figure 3.1.5.

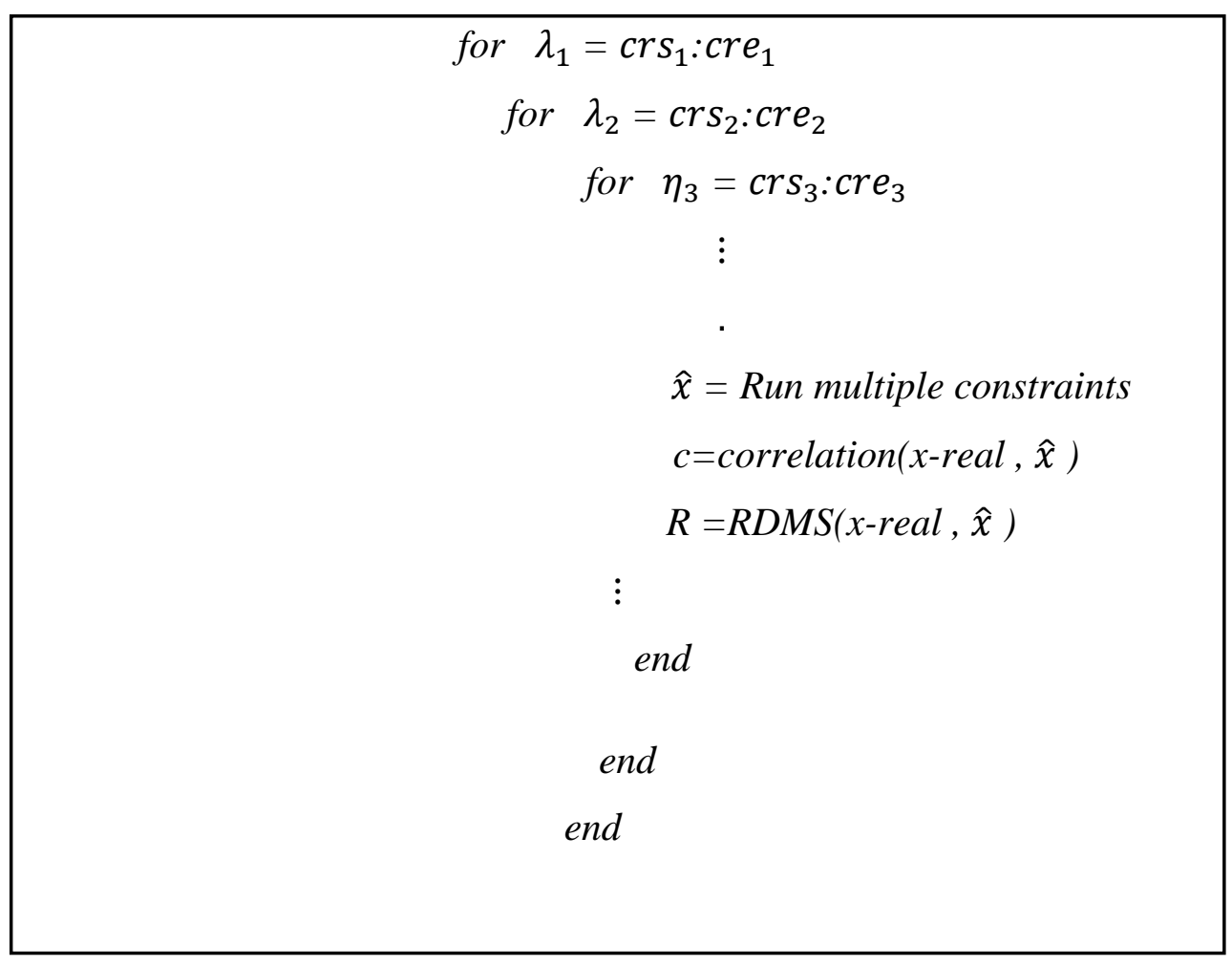

Figure 3.1.5: Pseudocode in finding the best solution

\subsubsection{Regularization Parameters Calculation Using Genetic Algorithm}

This method is a complex use of genetic algorithm to find the best solution to the problem. Before we explain how we use GA to select the regularization parameters, we first give a brief explanation of genetic algorithm [48]. 


\subsubsection{Introduction to GA}

Genetic algorithm is a search technique for handling complex problems it plays a significant role in engineering optimization problems. Genetic algorithms are based on the underlying genetic process in biological organisms and on the natural evolution principles of populations.

These algorithms process a population of chromosomes with three operations: selection, crossover and mutation.

There two general ways to use genetic algorithm:

- Binary Coded Genetic Algorithms

- Real Coded Genetic Algorithms.

The chromosomes in Binary Coded Genetic Algorithms are binary numbers. In this study we used Real Coded Genetic Algorithms that use real valued numbers for chromosomes [48].

A GA starts with a population of randomly generated chromosomes, which is called the initial population.

During iterations, which are called generations, chromosomes in the population are evaluated by the fir function and rated for their success as solutions, and on the basis of these evaluations, a new population of chromosomes is formed using a random selection mechanism and the genetic operators such as crossover and mutation.

\subsubsection{Crossover Operator}

The crossover operator combines the features of two parent chromosomes to form offspring's, with the possibility that good chromosomes may generate better chromosomes and the bad chromosomes disappear from the population. The crossover operator is a method for sharing information between chromosomes and it 
is not usually applied to all chromosomes in the population. A random choice is made, where the likelihood of crossover being applied depends on probability defined by a crossover rate, the crossover probability.

\subsubsection{Mutation Operator}

To increase the structural variability of the population in hand the mutation operator arbitrarily alters one or more components, genes, of a selected chromosome. By using mutation in GAs lost or unexplored genetic material is restored into the population to prevent the premature convergence of GA to suboptimal solutions; it insures that the probability of reaching any point in the search space is never zero.

\subsubsection{Fit function}

The fit function determines the optimality of a solution. An ideal fit function correlates closely with the algorithm's goal and directs the populations so a global minimum is reached. Speed of evaluation is very important, as a typical genetic algorithm must be iterated many, many times in order to produce a usable result. Therefore one of the most important aspects of a convergent genetic algorithm is to determine a fit function such that the solution does not get stuck in local minima or that the program does not get lost, which is commonly the case when using other optimization methods. Thus definition of the fit function is not straightforward in many cases and is often performed iteratively if the fittest solutions produced by GA are not the desired solutions. 


\subsubsection{Using GA for Regularization Parameter Selection}

Figure 3.1.6 summarizes the first steps taken to start the GA.

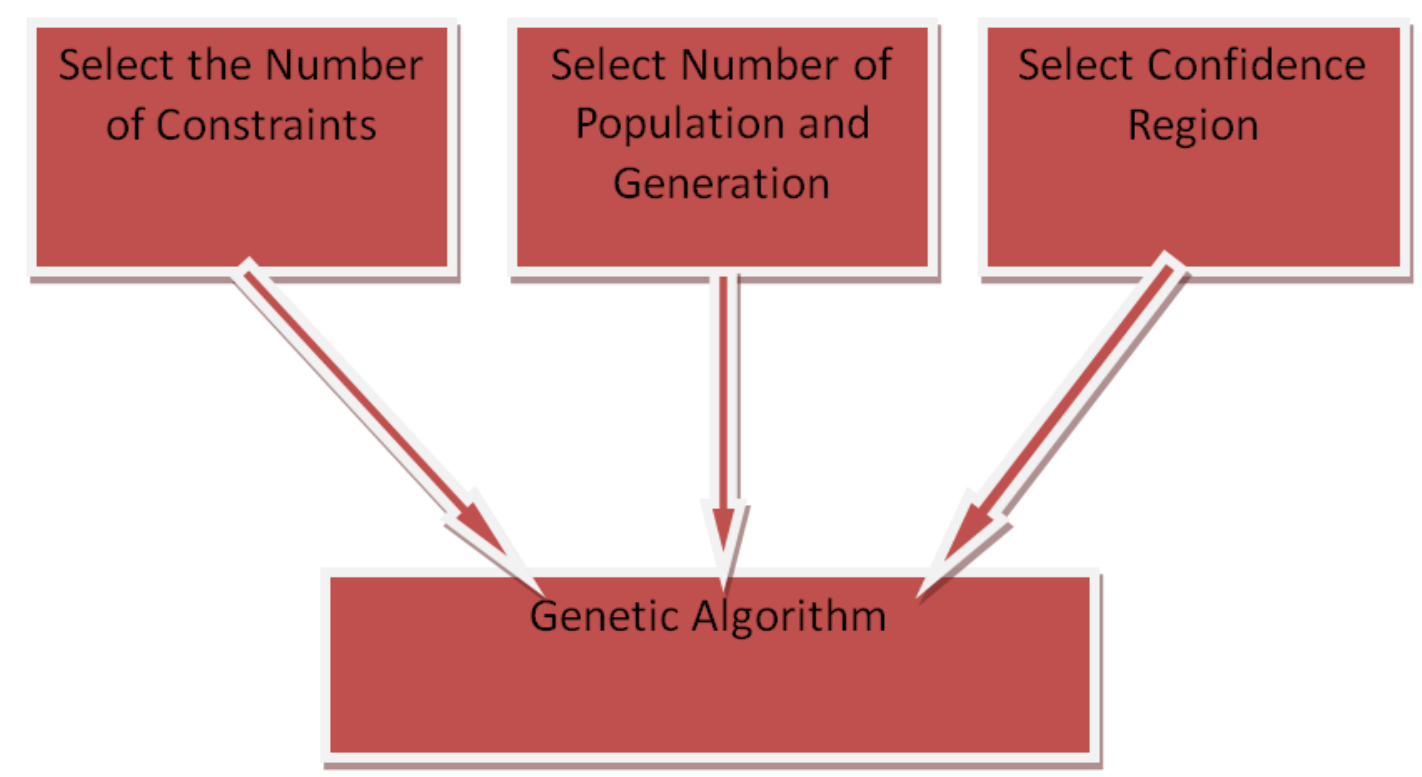

Figure 3.1.6: Flow chart of the inputs needed for regularization parameter calculation using GA.

Confidence Region Selection: Before we start the search for the optimum parameters, we choose a region that we assume the regularization parameters most likely belong to. This is similar to the confidence range of the maximum CC approach. By choosing a correct confidence region we decrease the time consumed to detect the parameters. Although if the confidence region is not selected correctly the GA method by using the mutation property does converge to a correct minimum, however, the time required for the algorithm to converge is increased significantly. If the confidence region that is selected is large, the same problem occurs; the population size should be increased to increase the chance of finding a good gene.

Number of Constraints: To start the search for optimum regularization parameters, we first input the number of constraints that we are going to use in the solution of the inverse problem. These parameters should be utilized along with their 
corresponding constraint norms in the fit function calculation. In the results given in the next chapter, two regularization parameters are used; therefore any chromosome consists of two parameters.

Number of Population and Generation: The population number determines the number of random chromosomes that are to be taken from the confidence region, and the number of chromosomes that are to be generated in each generation. Therefore, by increasing population number, the time required for the program to run is increased because the fit function for each chromosome should be calculated. Generation number determines the number of new populations that are to be created by mutation and cross over, at each iteration. Therefore, in total the number of fit function evaluations equals the population size multiplied by the generation number.

It is important to note that each chromosome is a row of the population matrix therefore for $\mathrm{N}$ numbers of constraints the population matrix is $N \times \mathrm{PS}$ where PS is the population size. For example the population for two constraints is Figure 3.1.7.

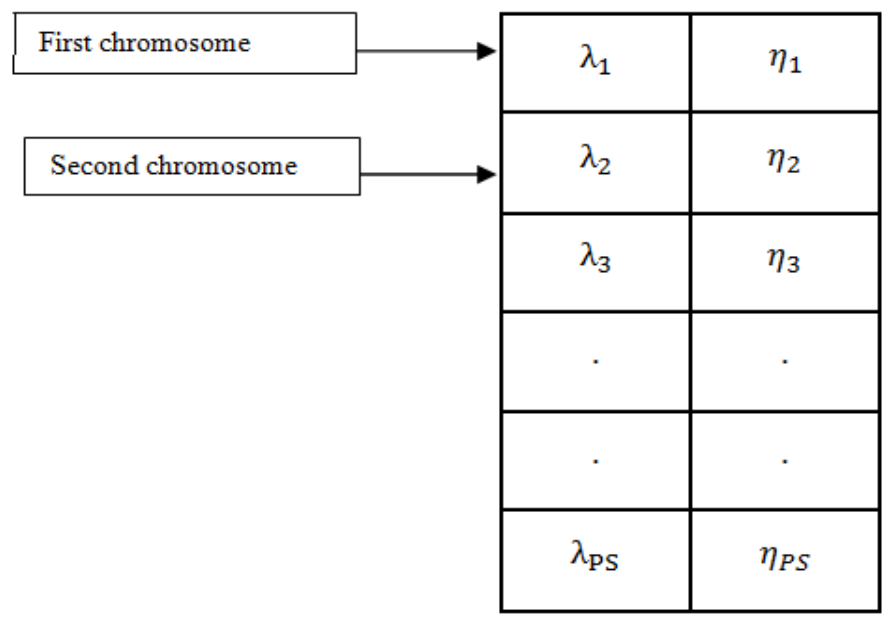

Figure 3.1.7 Population for two constraints. 


\subsubsection{Fit Function for Regularization Parameter Calculation}

To calculate the fit function for each chromosome we need to find the solution for each chromosome, therefore a multiple constraint solution is carried out for each chromosome (i.e., each row of the population matrix shown in Figure 3.1.7). Thus for each chromosome, one of the procedures explained in sections 3.3.1 or 3.3.2 is carried out.

After determining a solution for each chromosome, a fit value is evaluated. The fit function that we proposed for this problem is inspired by the L-curve function:

$$
\text { Fit function }=\frac{\|A \widehat{x}-Y\|^{2}}{\lambda^{2}\|\widehat{x}\|^{2}}+\frac{\|A \widehat{x}-Y\|^{2}}{\eta^{2}\|\widehat{x}\|^{2}}+\lambda^{2}\|R \widehat{x}\|^{2}+\eta^{2}\|\Gamma \widehat{x}\|^{2}
$$

The fit function given in equation 3.13 is for two constraints but as it is obvious this could be upgraded to more the two constraints.

The main objective using this fit function is to calculate a solution using the algorithms introduced in sections 3.3 .1 and 3.3.2 to minimize the residual norm $\|A \widehat{x}-Y\|$. Although we are trying to minimize $\|A \widehat{x}-Y\|$, the solutions obtained to satisfy this equation could produce unreasonable results with very large or very small norms. Therefore, to compensate solutions with large norms, we added the resultant norm $(\|R \widehat{x}\|)$ to the fit function value so solutions with large norms would produce large fit functions. Solutions with very small norm would produce a large value for $\frac{1}{\|\widehat{x}\|}$, therefore the fit function value would increase and chromosomes would be considered as bad chromosomes.

After a fit value for each chromosome has been evaluated, the chromosomes are ranked from the lowest fit value to the largest fit value. Next, by using mutation and crossover properties, new populations are created in way that the smaller the fit value the more chance there is for the chromosome to undergo crossover therefore the chromosomes with small fit values have the chance to transfer their genes to the next 
generation and the chromosomes with large fit values are less likely to reproduce. By this way the new generation has better chromosomes then the previous generation and a new iteration is started until the best solution can be achieved.

\subsubsection{Real Valued Crossover}

To obtain new populations in a search for better chromosomes the crossover and mutation operators are used. In crossover operation, two chromosomes are chosen from the available population; these are called as the father and the mother chromosomes Figure 3.1.8. Note that the better the chromosome is, the more chance it has to be selected. In another words, chromosomes with smaller fit functions have more probability to be selected. This is one way of passing better genes to the next generations.

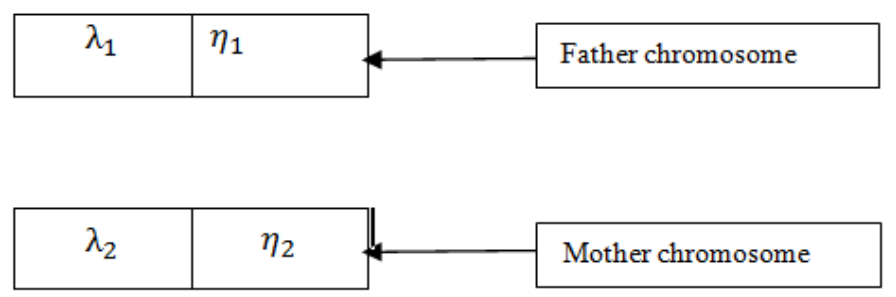

Figure 3.1.8: Father and mother chromosomes

In the first step of the crossover operation, the child chromosome is equated to the father chromosome (Figure 3.1.9).

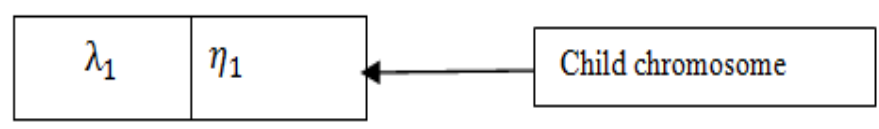

Figure 3.1.9: child chromosome before crossover

As the second step, one index of the father chromosome is selected randomly; in the example illustrated here, we have two indices, one corresponding to $\lambda$, and another 
corresponding to $\eta$. For example, in Figure 3.1.10, index two is selected randomly, thus $\eta$ is changed to a new value defined by the relation:

$$
\eta_{\text {child }}=(1-\propto) \eta_{1}+\propto
$$

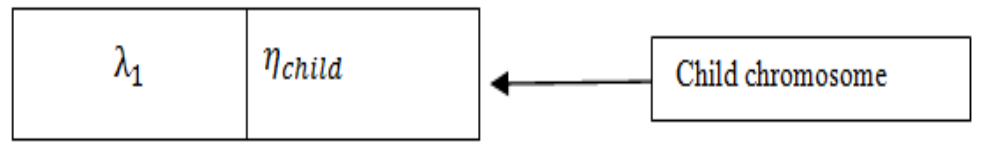

Figure 3.1.10: child chromosome after crossover.

Where $\propto$ is a selected random number. Graphically, we explain this update as selecting a random point between two $\eta_{1}$ and $\eta_{2}$ values in Figure 3.1.11.

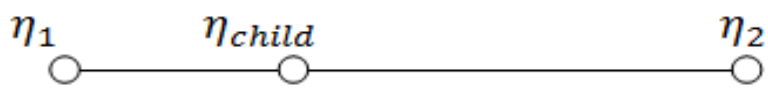

Figure 3.1.11: liner interpolation

The same procedure would be carried out for $\lambda$, if the first index was selected at the beginning.

\subsubsection{Real Valued Mutation}

As mentioned in section 3.4.1.2, by using the mutation property, we prevent the premature convergence of GA to suboptimal solutions. We have previously stated that the chromosomes with smaller fit values have more chance to pass on to the next generations, but this could cause the system to move to a local minimum and a global minimum could never be reached. Therefore, there is always a need to produce chromosomes from different regions. The mutation operator is similar to crossover, 
but in this case after choosing a chromosome, the parameter with the randomly selected index is changed by adding or substituting a random number so that lost or unexplored genetic material are returned into the next population:

$\eta_{\text {child }}=\eta_{\text {father }}+(\propto-0.5) \times \mathrm{MF} \times \mathrm{CRL}$

Where $\propto$ is a random number selected from [0 1] region and MF equals:

$\mathrm{MF}=\exp \left(-\right.$ current generation Count $\left.\times \frac{10.0}{\text { generation Count }}\right)$

$\mathrm{CRL}=$ Confidence Region Length

Any chromosome in the population can undergo mutation and the value of fit function is not important all the chromosomes share the same probability to undergo mutation which is the mutation probability. The mutation probability could either kept constant or it can be can be arranged in such way that it would decreases in each generation so in earlier generations there are more chance of being mutated this is so the algorithm can take samples from any point of the confidence region and regions close to it. The mutation probability designed for this problem is as equation

$M P=\frac{1}{\text { current generation } \times \frac{\text { number of parameters }}{\text { total generation }}+2}$

After this procedure is over the new generation is produced. In the next generation all this events are gone through again until the stopping criteria is reached this criteria is either when the number of total iterations are reached which in this case the algorithm represents the best chromosome of all populations as the solution, or the genetic diversity of the populations are zero and all the chromosomes in the populations are converged to a single chromosome which in this case this chromosome is introduced as the solution which is mostly the case. 


\section{CHAPTER 4}

\section{RESULTS AND DISCUSSION}

In the following chapter first the data used for simulations are explained and then the reconstruction results for inverse problem of electrocardiography are given.

\subsection{Test Data}

The body surface potential measurements used in this study are simulated using the QRS intervals of measured epicardial potentials.

The epicardial potentials used for this study are measured at University of Utah Nora Eccles Harrison Cardiovascular Research and Training Institute (CVRTI) [36]. The measurements were taken from dog hearts, which were perfused from another dog's circulatory system and suspended in an electrolytic filled (500 $\Omega \mathrm{cm}$ ) adolescence human thorax shaped fiberglass tank. To measure the epicardial potentials a nylon sock electrode with silver wires was slipped over the ventricles. During measurements the heart is stimulated from the ventricles to simulate ventricular arrhythmias. The epicardial measurements are taken from 490 points with sampling rate 1000 sample per second.

The forward transfer matrix that maps epicardial potentials to body surface potentials was calculated using boundary element method (BEM), The forward solver is 
developed by Dr. Özlem Birgül in Middle East Technical University (METU). The thorax model used for simulation of torso potentials consists of heart, torso and lungs.

The forward transfer matrix is used to obtain 771 body surface potentials form epicardial potential measurements from 490 points respectively.

The body surface potentials are obtained from the epicardial potential measurements by a multiplication with the transfer matrix and addition independent and identically distributed Gaussian noise Figure 4.1.

To avoid Inverse crime, the A matrix used in inverse solution is a transfer matrix calculated using only heart and torso geometric information (i.e., homogeneous forward problem solution).

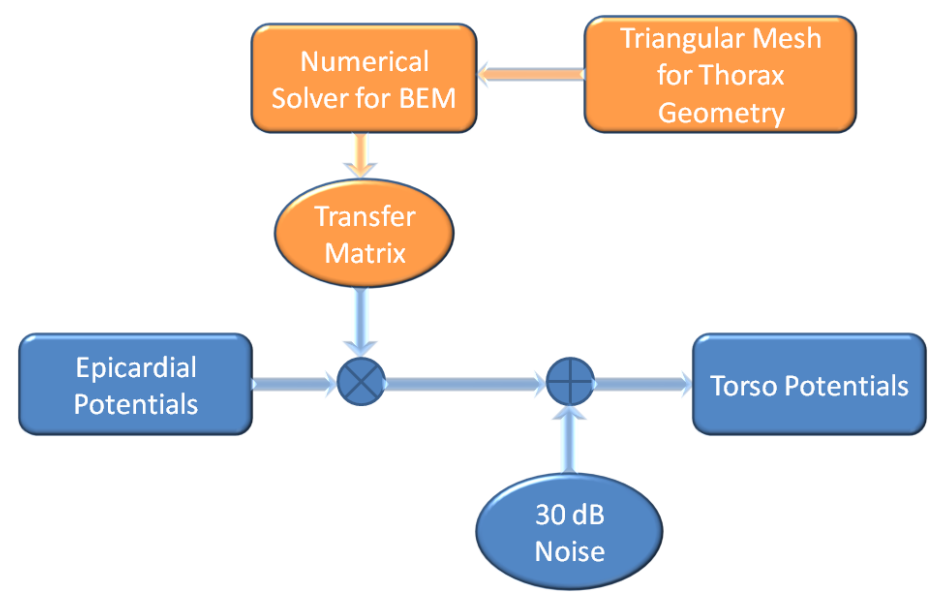

Figure 4.1: Torso potentials calculation

\subsection{Quantitative Comparisons:}

The quantitative comparisons of the solutions are made using Correlation Coefficients (CC) and Relative Difference Measurement Star (RDMS), 


$$
\mathrm{CC}(\mathrm{t})=\frac{\mathrm{N}\left(\sum_{\mathrm{i}=1}^{\mathrm{n}} \mathrm{X}_{\mathrm{i}}(\mathrm{t}) \widehat{\mathrm{X}}_{\mathrm{i}}(\mathrm{t})-\left(\sum_{\mathrm{i}=1}^{\mathrm{n}} \mathrm{X}_{\mathrm{i}}(\mathrm{t})\right)\left(\sum_{\mathrm{i}=1}^{\mathrm{T}} \widehat{\mathrm{X}}_{1}(\mathrm{t})\right.\right.}{\sqrt{\left.\mathrm{N}\left(\left(\sum_{\mathrm{i}=1}^{\mathrm{n}} \mathrm{X}_{\mathrm{i}}^{2}(\mathrm{t})\right)\left(\sum_{\mathrm{i}=1}^{\mathrm{n}} \mathrm{X}_{\mathrm{i}}(\mathrm{t})\right)^{2}\right)\right)} \sqrt{\mathrm{N}\left(\sum_{\mathrm{i}=1}^{\mathrm{n}} \widehat{\mathrm{X}}_{\mathrm{i}}^{2}(\mathrm{t})\right)\left(\sum_{\mathrm{i}=1}^{\mathrm{n}} \widehat{\mathrm{X}}_{\mathrm{i}}(\mathrm{t})\right)^{2}}}
$$

$$
\operatorname{RDMS}(\mathrm{t})=\sqrt{\sum\left[\frac{\widehat{X}_{l}(\mathrm{t})}{\sum_{i=1}^{T n} \hat{X}_{i}^{2}(\mathrm{t})}-\frac{X_{i}(\mathrm{t})}{\sum_{i=1}^{n} X_{i}^{2}(\mathrm{t})}\right]^{2}}
$$

An increase in CC or a decrease in RDMS value is interpreted as an improvement in the method.

Although RDMS and CC give us quantitative information about the performances of the methods used for inverse electrocardiography problem, it is also important to compare original and reconstructed epicardial distributions visually. One of the reasons for this visual comparison is that the error measures used in this study cannot put necessary emphasis on true reconstruction of activation wave front, which is one of the most important features for inverse electrocardiography problem. The software that we used in this study is MAP3D, which is developed at Utah University, Nora Eccles Harrison Cardiovascular Research and Training Institute for the purpose of displaying and editing three-dimensional geometric models and time-based data associated with those models.

The results are displayed as isopotential surfaces of epicardial potential distribution on the 3D heart geometry.

While the results are compared visually, two important characteristics are considered:

1. The proposed solution's ability to follow the wave front without dispersing it much. The dispersion in the wave front is due to the smoothing effects of regularization.

2. The solution's ability to correctly reconstruct the activated or inactivated regions. 


\subsection{Multiple Spatial Constraints}

In this section, we study the effects of utilizing more than one spatial constraint, as introduced in section (3.3.1). We use two spatial constraints; in equations 3.9 we choose the identity matrix as the first regularization matrix (i.e., the energy constraint), and the surface Laplacian as the second regularization matrix. This formulation requires two regularization parameters: $\lambda$, corresponding to the first constraint, and $\eta$, corresponding to the second one. We use two approaches for the selection of $\lambda$ and $\eta$,

1. Best CC approach, as explained in section 3.4.1,

2. Our proposed approach, i.e., GA-based approach, as explained in section 3.4.2.

The results obtained by these two regularization parameter selection approaches are compared with each other and with the results of zero-order Tikhonov regularization. All inverse solutions are also compared with the original epicardial potential distribution, using the quantitative and the qualitative measures introduced in Section 4.2.

The first data set we used is torso potentials with $30 \mathrm{~dB}$ Signal to Noise Ratio (SNR). Then we repeat the same procedure with the torso potentials simulated at 10dB SNR.

In the application of the GA method, we used a population size of 40 , and a generation size of 70 . Thus, the total number of fit function evaluations is 2800 , which takes about 240- 480 seconds, depending on the data size. 


\subsubsection{Two Spatial Constraints with $30 \mathrm{~dB}$ Noise Data}

In the following section Multiple Spatial (MS) method is applied to data with $30 \mathrm{~dB}$ SNR.

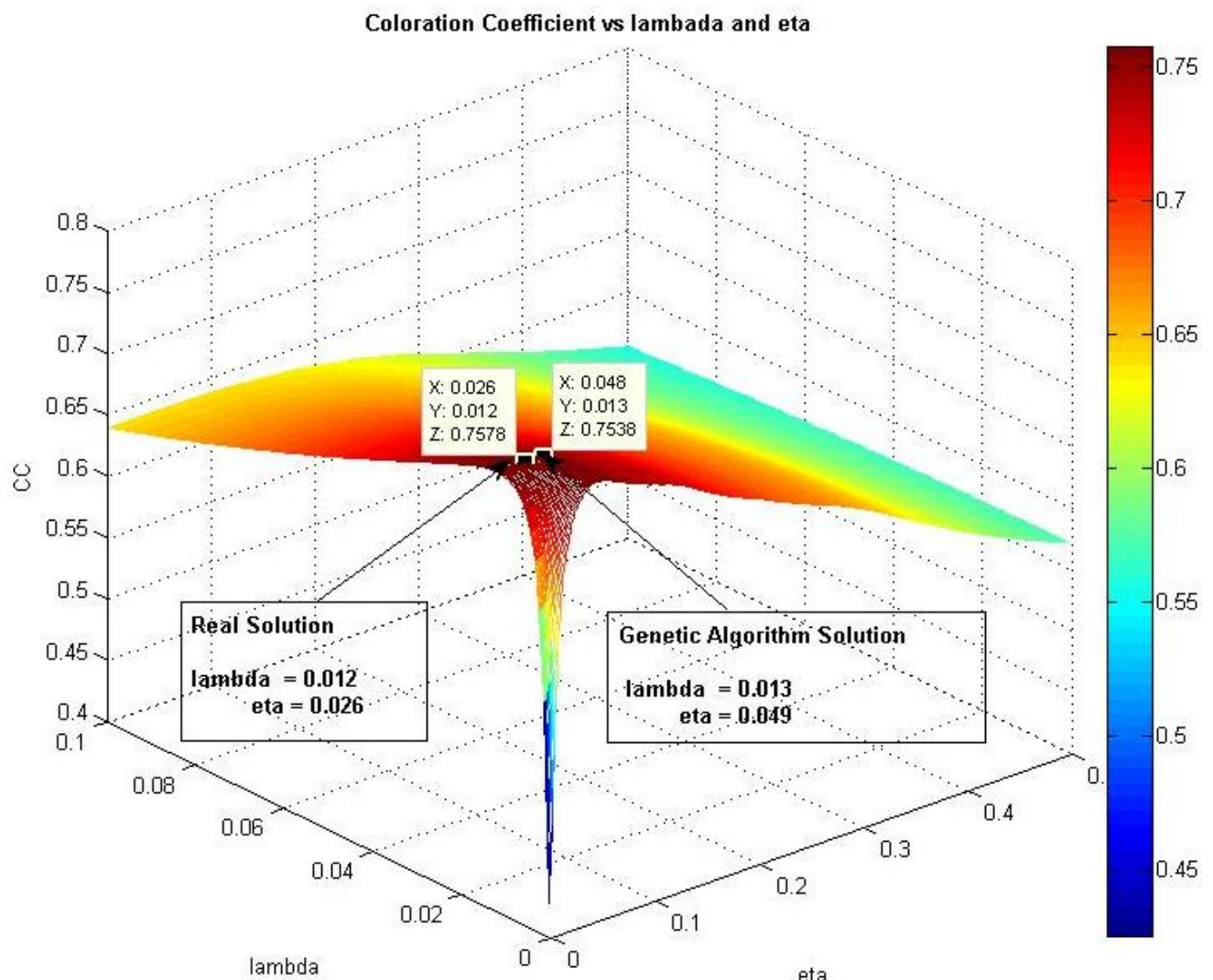

Figure 4.2 Correlation coefficient calculated for all $\boldsymbol{\lambda}$ and $\boldsymbol{\eta}$ values using MCC method

Figure 4.2 and similar figures demonstrated in this chapter are obtained by running the multiple constraints method explained in the sections 3.3.1 and 3.3.2 for a range of regularization parameters and by calculating the $\mathrm{CC}$ values corresponding to each $\lambda$ and $\eta$ pair. As stated before, in the MCC method, the regularization parameters are selected so that maximum correlation coefficient values could be achieved by the solution. In these figures, the regularization parameter pair calculated by using MCC approach is labeled as "Real Solution" and the solution to GA approch is labeld as "Genetic Algorithm Solution". 
As it could be seen from Figure 4.2 the parameters calculated by the GA method are very close to the parameters calculated using the MCC method. Therefore the solution obtained with the GA method parameters is also very close to the MCC method solution.

By looking at the average CC and RDMS values in Table 4.1, we observe that the regularization parameters chosen by the GA method produce very similar results with the MCC method.

Table 4.1: MCC and GA method solution results for $30 \mathrm{~dB}$ SNR data.

\begin{tabular}{ccc}
\hline 30 dB SNR data & GA method & Maximum CC method \\
\hline CC & $0.7538 \pm 0.0 .13$ & $0.7578 \pm 0.14$ \\
RDMS & $0.6455 \pm 0.15$ & $0.6407 \pm 0.15$ \\
$\boldsymbol{\lambda}$ value & 0.012 & 0.013 \\
$\boldsymbol{\eta}$ value & 0.026 & 0.048 \\
\hline
\end{tabular}

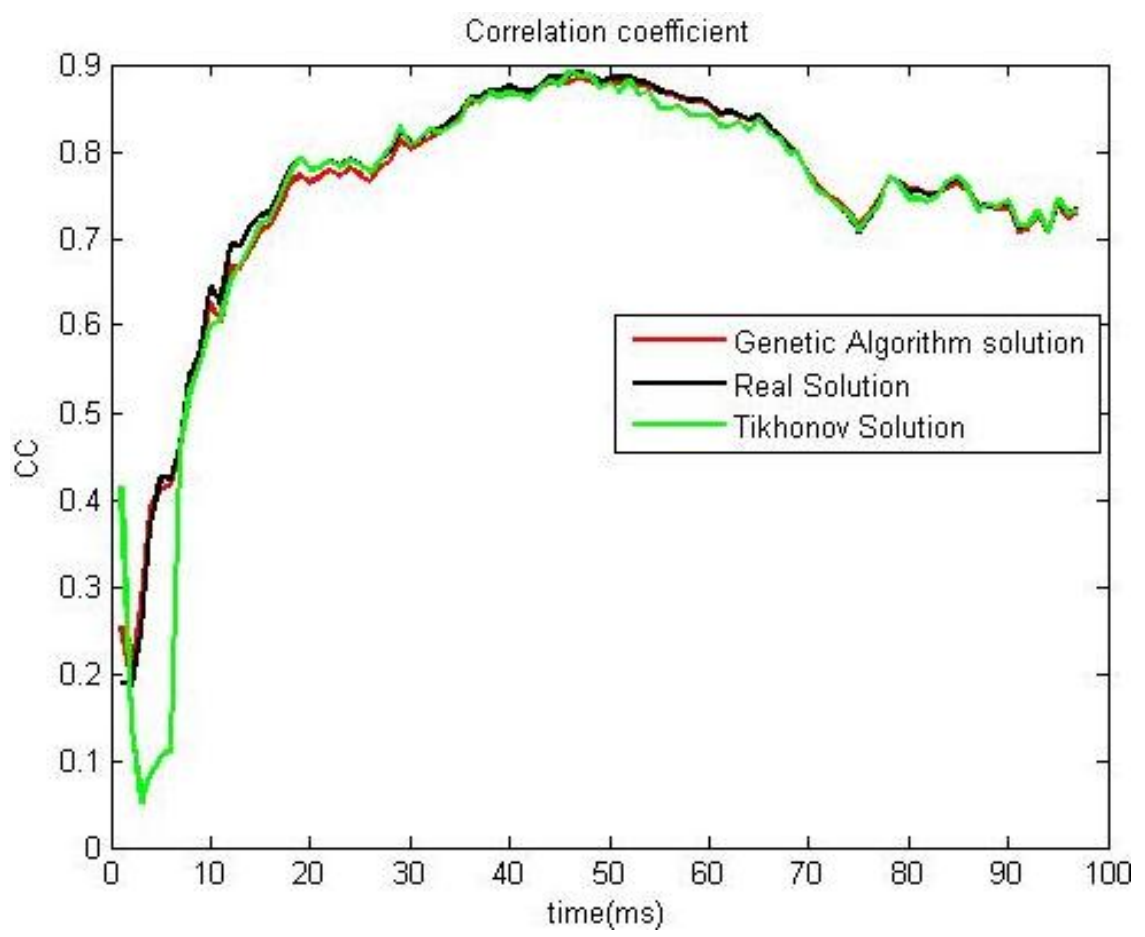

Figure 4.3: Correlation coefficient figure for each time instant. 


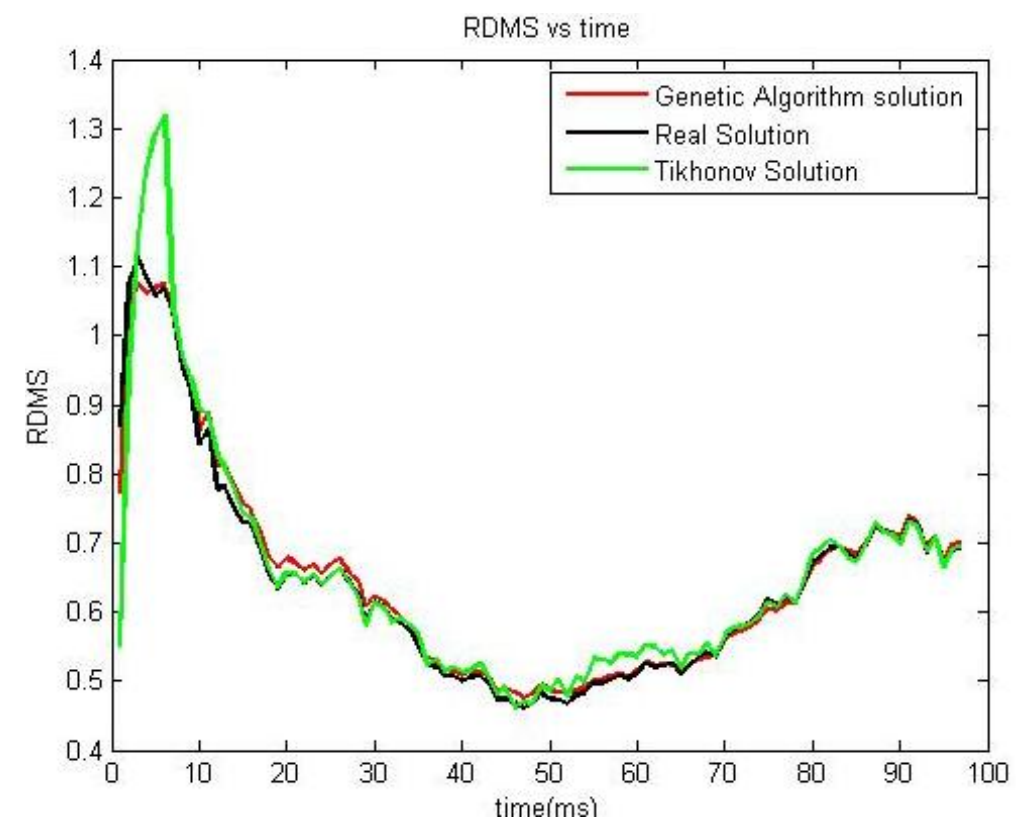

Figure 4.4 : RDMS figure for each time instant.

Figure 4.3 and Figure 4.4 shows the CC and RDMS values, respectively for all time instants during the QRS region for Tikhonov regularization, and for multiple spatial constraint approach with the parameters chosen by MCC and GA methods. These plots demonstrate that the solution of GA method follows the MCC solution. Performance of the Tikhonov regularization approach is poorer than the multiple constraint methods in the earlier time frames; but it recovers after about 10 seconds. The average values of CC and RDMS presented in Table 4.2, on the other hand, cannot differentiate between the results obtained by the Tikhonov regularization and the ones obtained by the two multiple constraints methods.

In Table 4.3 the results of the second data set are shown which are similar to the results given for Table 4.2 . 
Table 4.2 : Means and standard deviations of CC and RDMS values for $30 \mathrm{~dB}$

\begin{tabular}{lll}
\hline 30 dB SNR data & CC & RDMS \\
\hline Tikhonov & $0.74 \pm 13$ & $0.65 \pm 0.14$ \\
$\begin{array}{l}\text { Multiple constraints (genetic } \\
\text { algorithm) }\end{array}$ & $0.75 \pm 0.0 .13$ & $0.64 \pm 0.15$ \\
$\begin{array}{l}\text { Multiple constraints (maximum } \\
\text { cc) }\end{array}$ & $0.75 \pm 0.14$ & $0.64 \pm 0.15$ \\
\hline
\end{tabular}

Table 4.3: Means and standard deviations of CC and RDMS values for second data set

\begin{tabular}{lll}
\hline 30 dB SNR data & CC & RDMS \\
\hline Tikhonov & $0.76 \pm 0.12$ & $0.66 \pm 0.15$ \\
$\begin{array}{l}\text { Multiple constraints (genetic } \\
\text { algorithm) }\end{array}$ & $0.77 \pm 0.09$ & $0.64 \pm 0.12$ \\
$\begin{array}{l}\text { Multiple constraints } \\
\text { (maximum cc) }\end{array}$ & $0.78 \pm 0.09$ & $0.63 \pm 0.12$ \\
\hline
\end{tabular}



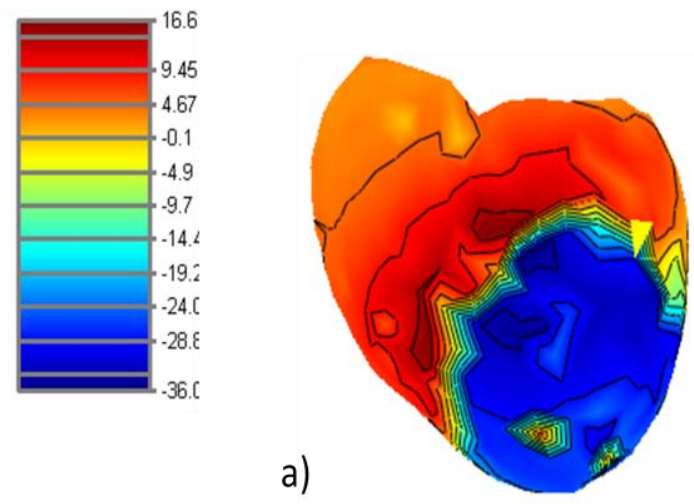

b)
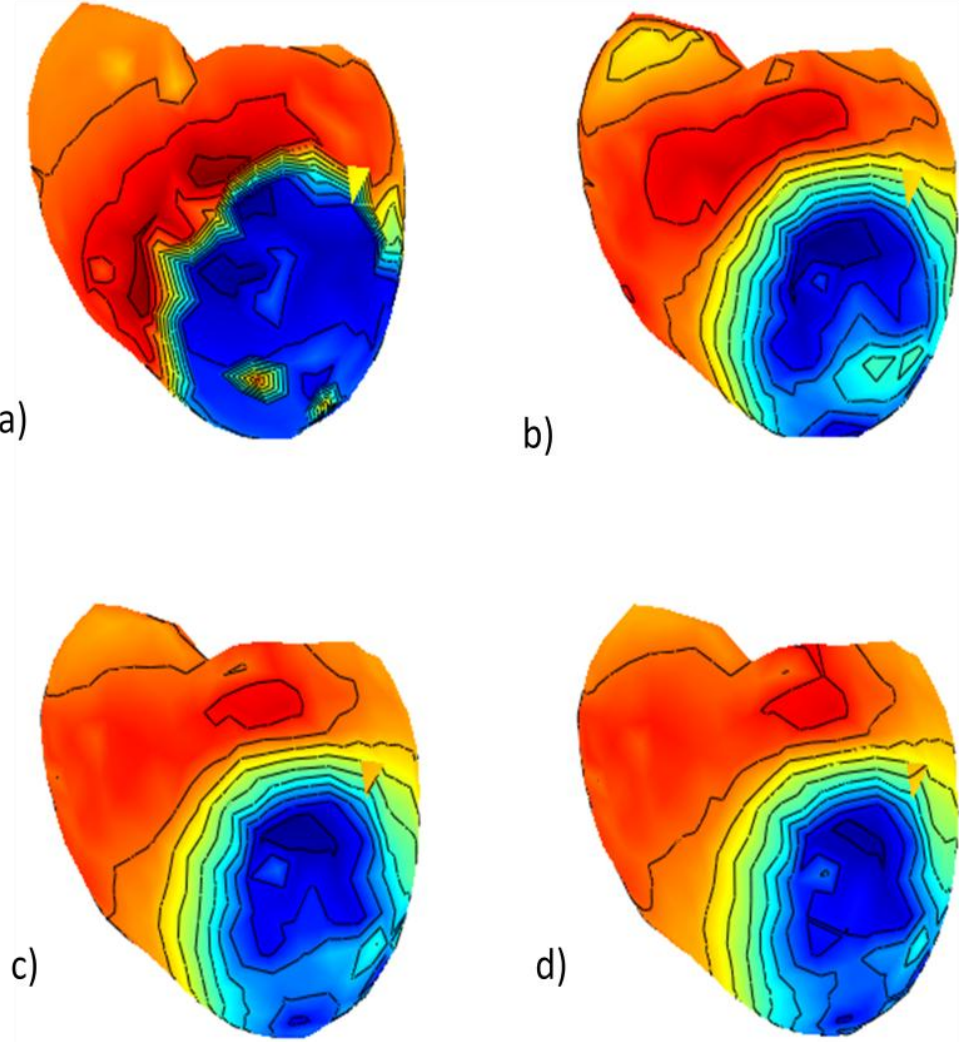

Figure 4.5: Epicardial potential map for 47th time instant. a) Real Epicardial Potentials, b) Tikhonov method, c) Multiple Constraints (MCC), d) Multiple Constraints (GA).

In Figure 4.5 , MAP3D images of epicardial potentials at the $47^{\text {th }}$ time instant are shown. Panels (a) to (d) show the original epicardial potential distribution, the epicardial potential reconstructed by Tikhonov method, the multiple spatial constraint solution using the MCC parameters, and the multiple spatial constraint solution using the GA parameters. In all the MAP3D results given in this chapter, this order of display is followed, unless stated otherwise. In all of the MAP3D images, the blue regions represent the already depolarized regions on the heart surface while the red regions show the regions at rest. At this time instant, the reconstructed epicardial potentials by all three methods are very similar to each other. 


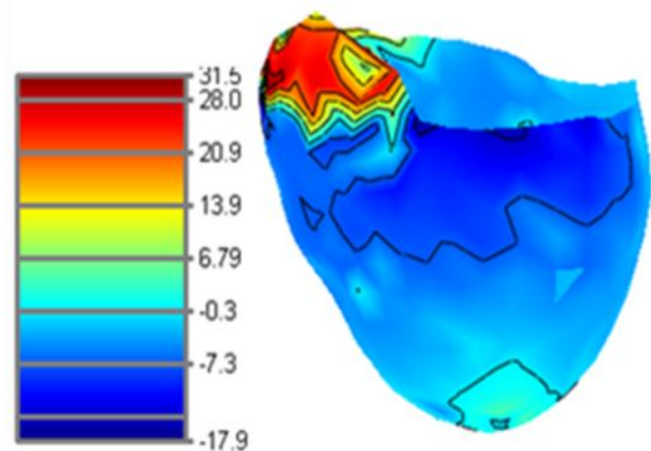

a)

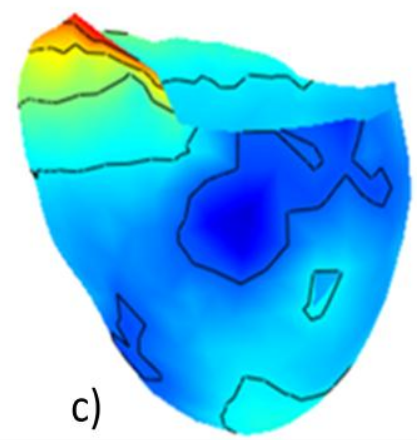

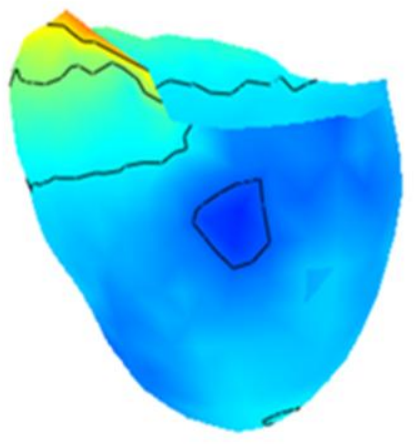

b)

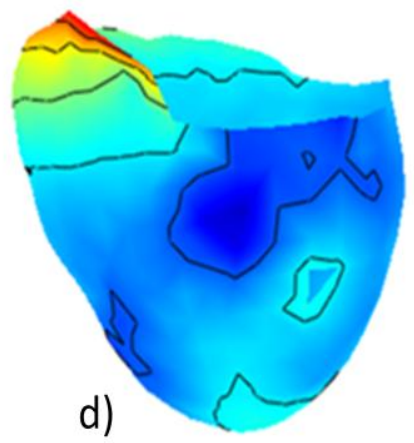

Figure 4.6: Epicardial Potential map for $91^{\text {th }}$ time instant. a) Real Epicardial Potentials, b) Tikhonov method, c) Multiple Constraints (MCC), d) Multiple Constraints (GA). 

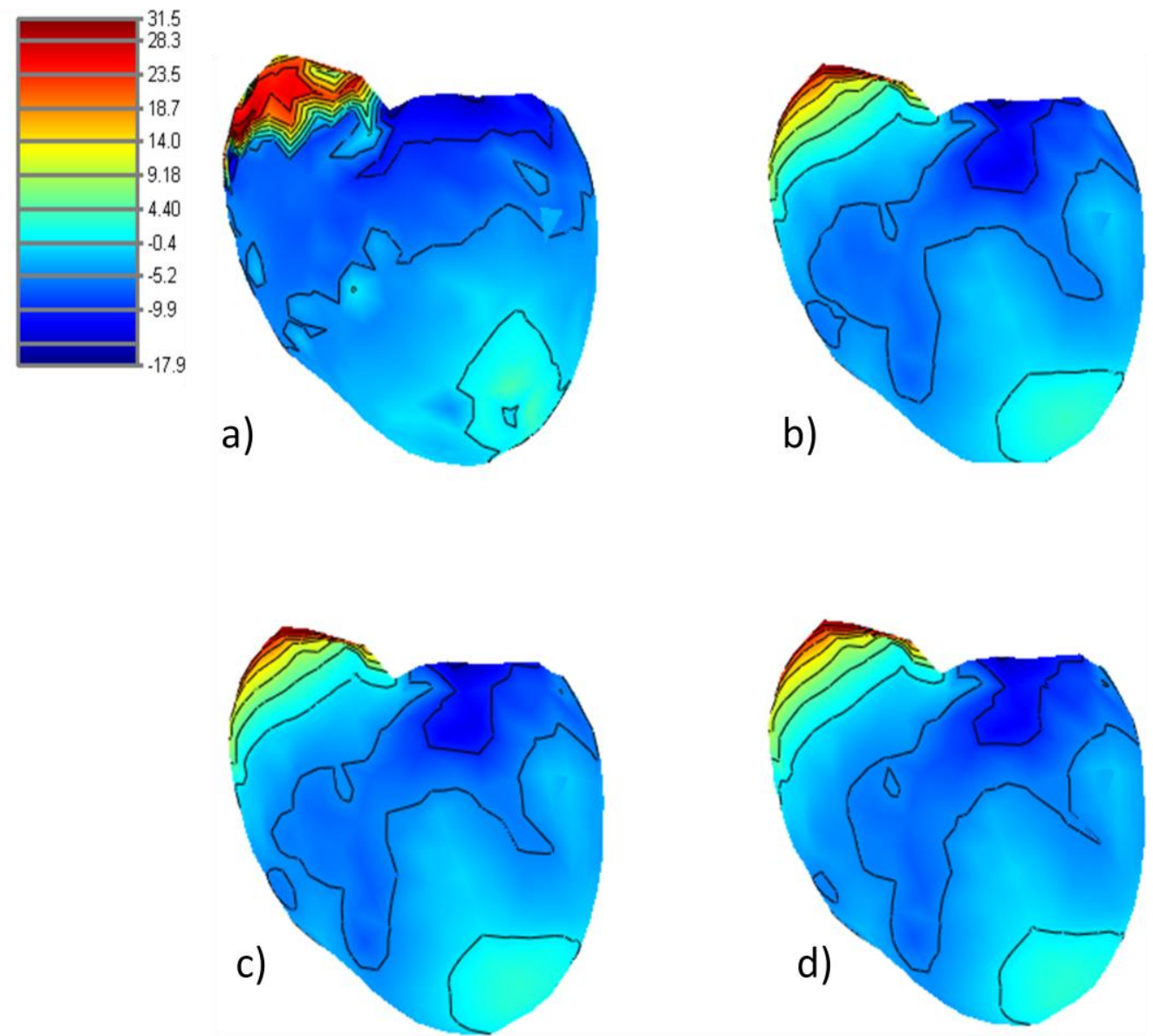

Figure 4.7: Epicardial potential map for $93^{\text {th }}$ time instant. a) Real Epicardial Potentials, b) Tikhonov method, c) Multiple Constraints (MCC), d) Multiple Constraints (GA).

In Figure 4.6 and Figure 4.7 the $91^{\text {th }}$ and $93^{\text {th }}$ time instant of epicardial potentials are demonstrated respectively. As it is expected the heart surface is covered by activated regions and there is a small region which is not activated yet the reconstructed epicardial potential have all detected the inactivated and the activated region and the solutions are very similar which is what we expected by looking at the previous tables and figures . 


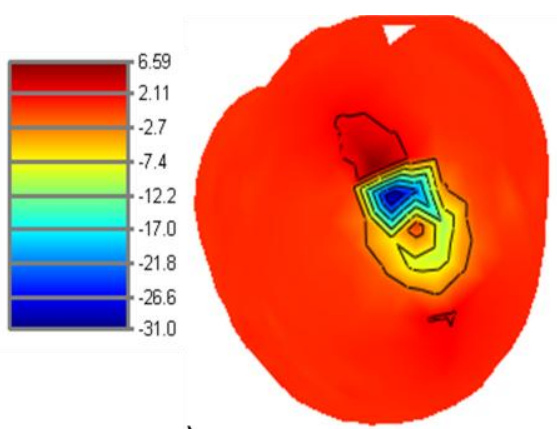

a)

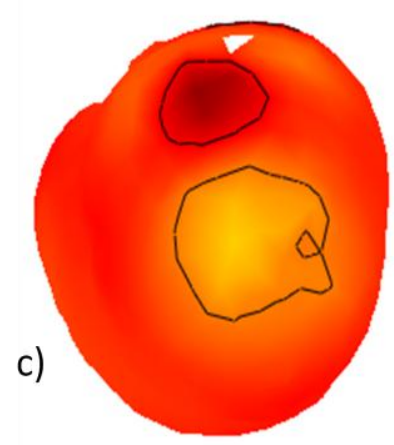

b)

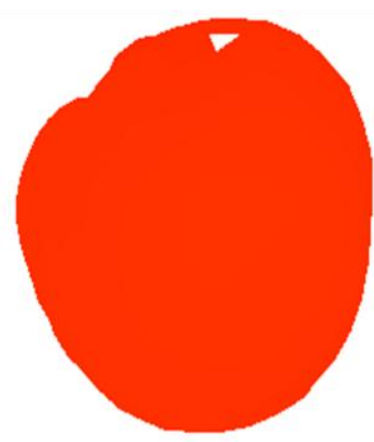

d)

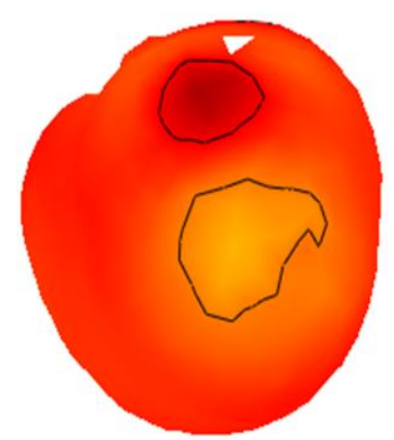

Figure 4.8 : Epicardial potential map for $6^{\text {th }}$ time instant. a) Real Epicardial Potentials, b) Tikhonov method, c) Multiple Constraints (MCC), d) Multiple Constraints (GA).

Figure 4.8 shows MAP3D original and reconstructed epicardial potential distributions, as labeled in the figure, at the $6^{\text {st }}$ time instant. We observe that the MCC and GA method has detected the ventricular ectopic focus, but the wavefront is smoother compared to the original distribution. The Tikhonov method, on the other hand, has failed to detect the ectopic focus at this time instant. 


\subsubsection{Two Spatial Constraints with $10 \mathrm{~dB}$ Noise Data}

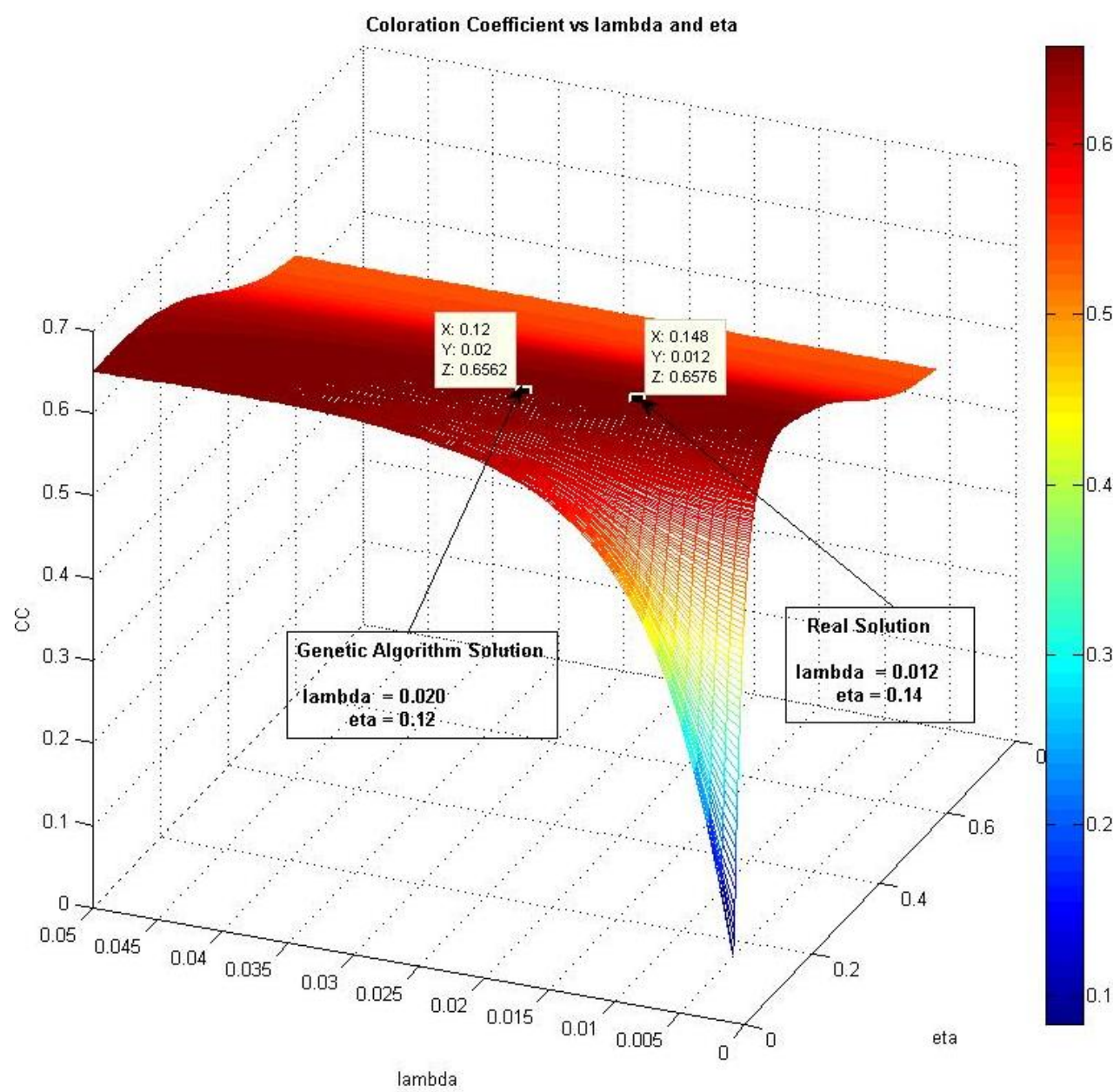

Figure 4.9: Correlation coefficient calculated for all $\boldsymbol{\lambda}$ and $\boldsymbol{\eta}$ values using MCC method for $10 \mathrm{~dB}$ data.

In this section we use torso data with $30 \mathrm{~dB}$ SNR which is calculated in the forward problem as shown in Figure 4.1.

It could be observed from Figure 4.9 that the regularization parameter values chosen by the GA method are very close to those chosen by the MCC method.

In Table 4.4 average $\mathrm{CC}$ and RDMS values are calculated for the multiple constraint solutions with regularization parameters chosen by the MCC and GA 
methods by examining this table, we can see that the solution calculated for the GA method is very close to the solution of the MCC method.

Table 4.4: MCC and GA method solution results for $10 \mathrm{~dB}$ SNR data

\begin{tabular}{ccc}
\hline $\mathbf{1 0}$ dB SNR data & GA method & Maximum CC method \\
\hline CC & $0.6562 \pm 0.22$ & $0.6576 \pm 0.21$ \\
RDMS & $0.7660 \pm 0.23$ & $0.7605 \pm 0.22$ \\
$\boldsymbol{\lambda}$ value & 0.020 & 0.020 \\
$\boldsymbol{\eta}$ value & 0.12 & 0.14 \\
\hline
\end{tabular}

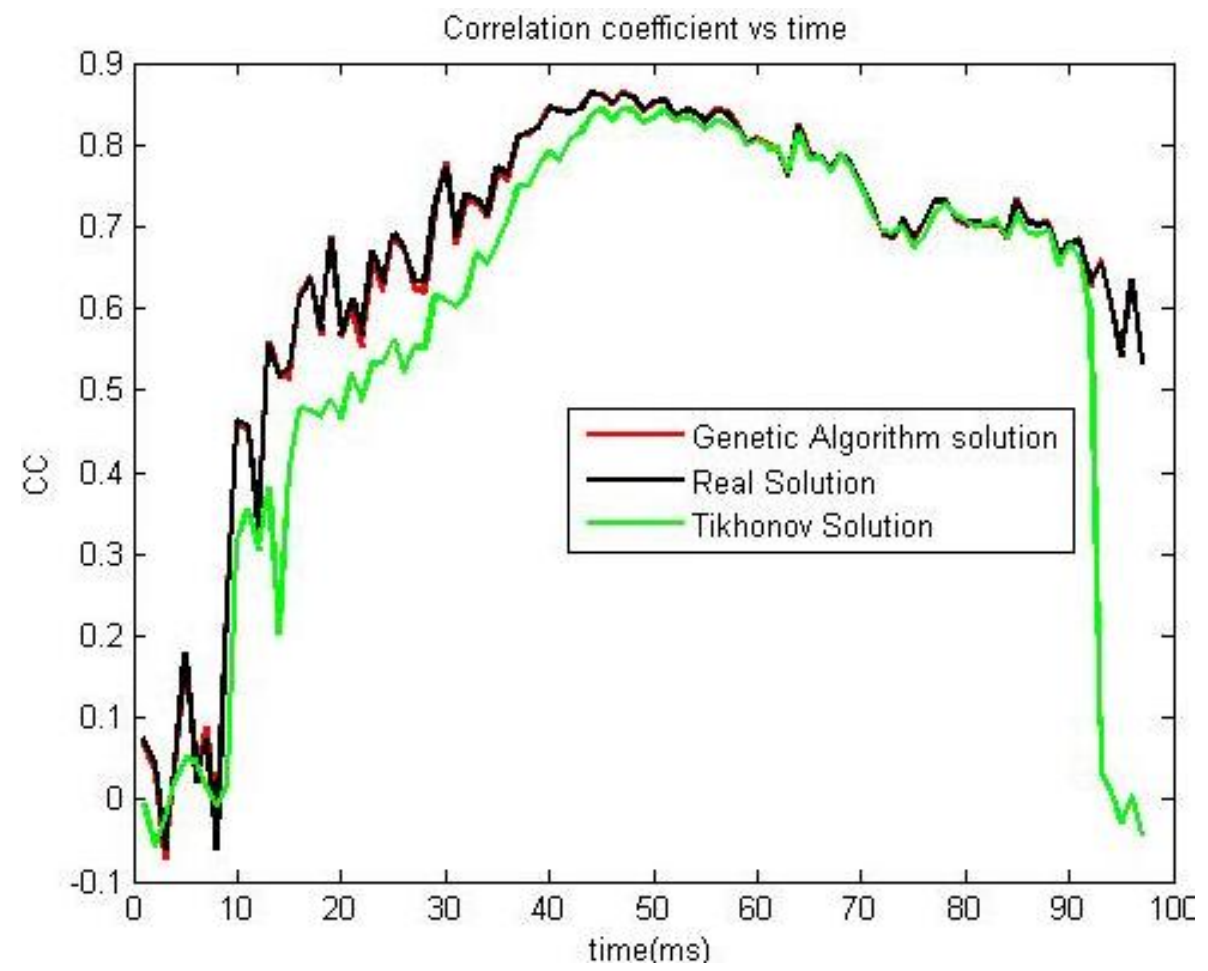

Figure 4.10: Correlation coefficients figures for each time instant. 


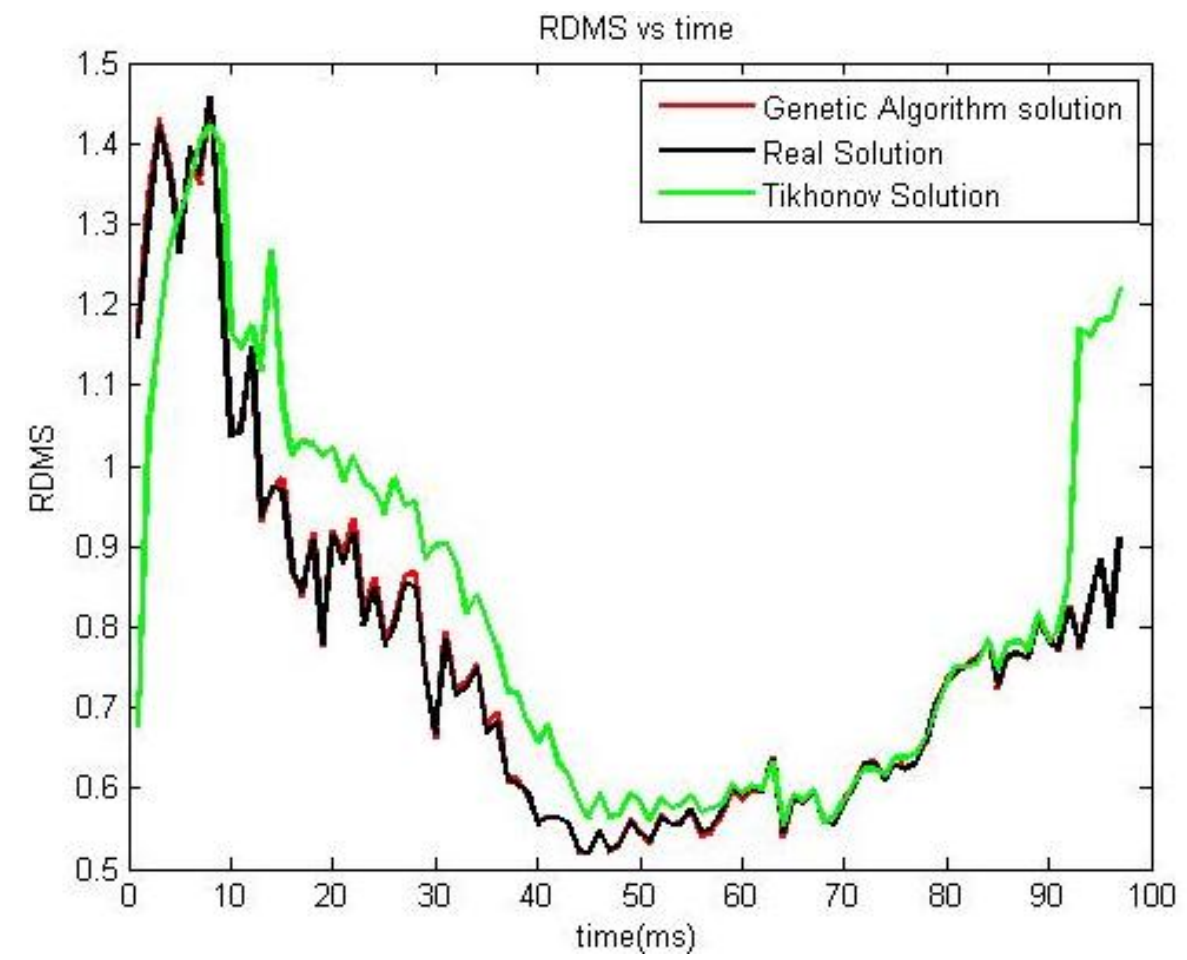

Figure 4.11: RDMS figures for each time instant

Figure 4.10 and Figure 4.11, show the CC and RDMS values respectively for all time frames, for the Tikhonov method and the two multiple constraint approaches. We can see that the GA method can successfully follow the MCC solution. Tikhonov solution, on the other hand, performs worse than the multiple constraint approaches. This fact is also supported by the average CC and RDMS values listed in Table 4.5; the Tikhonov method has an average $\mathrm{CC}$ value of $0.58 \pm 0.27$ while the GA method has an average $\mathrm{CC}$ value of $0.65 \pm 0.22$. Here we can see that the GA method has converged to the global minima, which is close to the solution obtained by the MCC method. 
Table 4.5: Means and standard deviations of CC and RDMS values

\begin{tabular}{lll}
\hline 10 dB SNR data & CC & RDMS \\
\hline Tikhonov & $0.58 \pm 0.27$ & $0.82 \pm 0.24$ \\
$\begin{array}{l}\text { Multiple constraints (genetic } \\
\text { algorithm) }\end{array}$ & $0.65 \pm 0.22$ & $0.76 \pm 0.23$ \\
$\begin{array}{l}\text { Multiple constraints } \\
\text { (maximum cc) }\end{array}$ & $0.65 \pm 0.21$ & $0.76 \pm 0.22$ \\
\hline
\end{tabular}

Table 4.6 demonstrates the results for a second test data set; the conclusions are the same as those made from Table 4.5.

Table 4.6 : Means and standard deviations of CC and RDMS values for second data set.

\begin{tabular}{lll}
\hline 10 dB SNR data & CC & RDMS \\
\hline Tikhonov & $0.62 \pm 0.24$ & $0.81 \pm 0.27$ \\
$\begin{array}{l}\text { Multiple constraints(genetic } \\
\text { algorithm) }\end{array}$ & $0.70 \pm 0.19$ & $0.73 \pm 0.21$ \\
$\begin{array}{l}\text { Multiple } \\
\text { constraints(maximum cc) }\end{array}$ & $0.71 \pm 0.17$ & $0.70 \pm 0.18$ \\
\hline
\end{tabular}



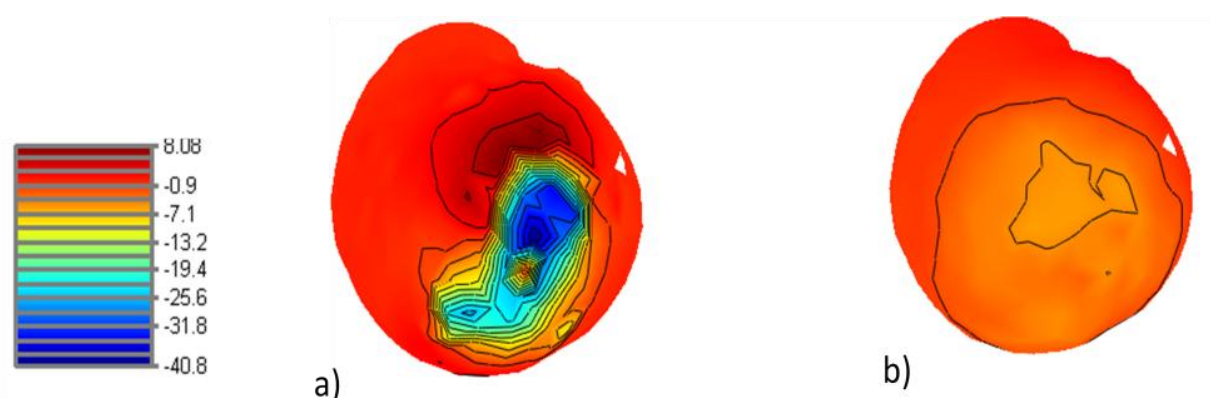

b)
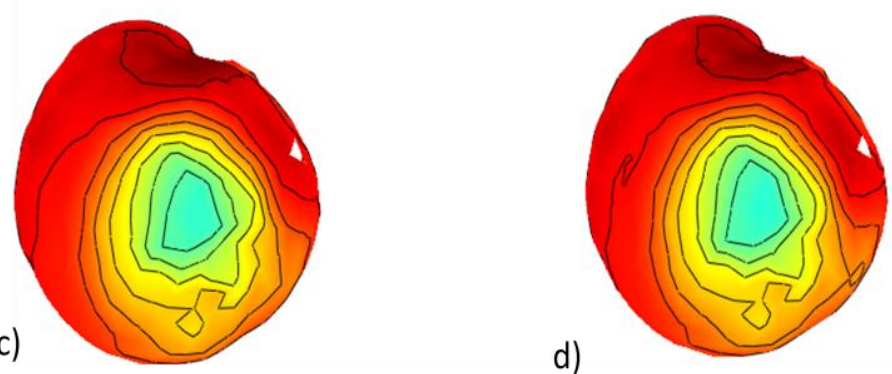

Figure $4.12: 21^{\text {th }}$ time instant of the epicardial potentials. a) Real Epicardial Potentials, b) Tikhonov method, c) Multiple Constraints (MCC), d) Multiple Constraints (GA).

Figure 4.12 shows MAP3D original and reconstructed epicardial potential distributions, as labeled in the figure, at the 21st time instant. We observe that the MCC method has detected the ventricular ectopic focus, but the wavefront is smoother compared to the original distribution. The epicardial map for the GA method is also very similar to the MCC solution. The Tikhonov method, on the other hand, has failed to detect the ectopic focus at this time instant. By looking at Figure 4.10 and Figure 4.11, we can see that this time instant is from an interval where CC is small and the RDMS is large with the Tikhonov method. Therefore we conclude that GA method is successful even in detecting earlier activated regions. 


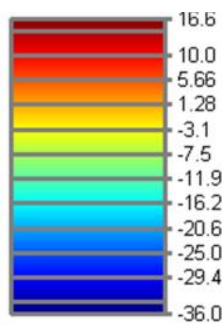

a)

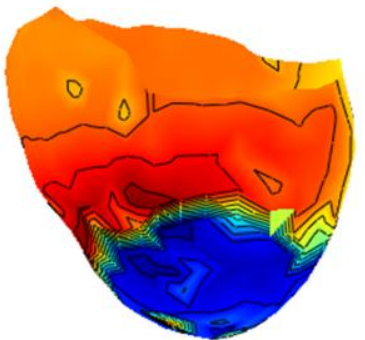

c)

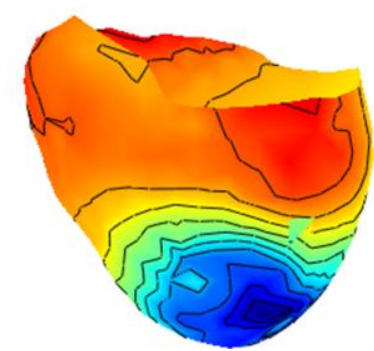

b)

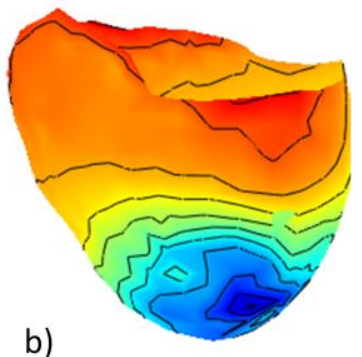

d)

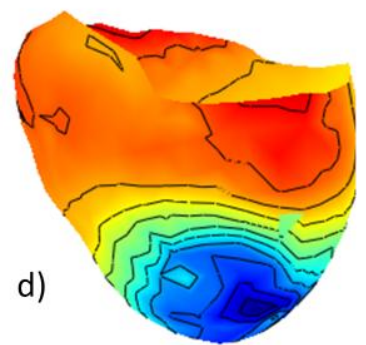

Figure 4.13: Epicardial potential map $10 \mathrm{~dB}$ SNR (47th time instant). a) Real Epicardial Potentials, b) Tikhonov method, c) Multiple Constraints (MCC), d) Multiple Constraints (GA).

In Figure 4.13, the epicardial potentials at the $47^{\text {th }}$ time instant are demonstrated. At this time instant, the Tikhonov method also produces results comparable to the multiple spatial constraint solutions, MCC and GA. By looking at Figure 4.10 we can see that at this time instant, Tikhonov, MCC and GA methods produce results with similar CC values. 


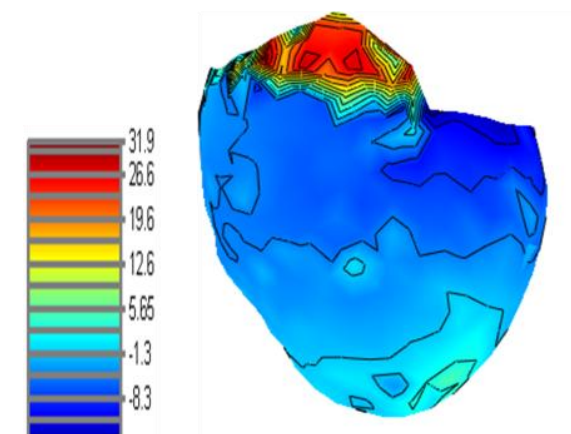

a)

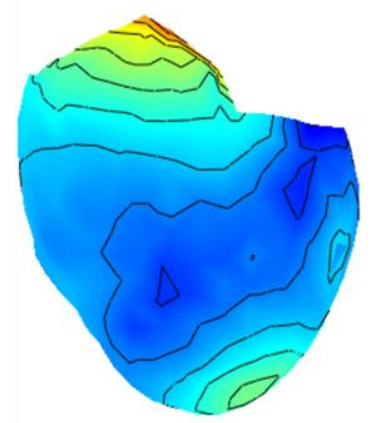

c)

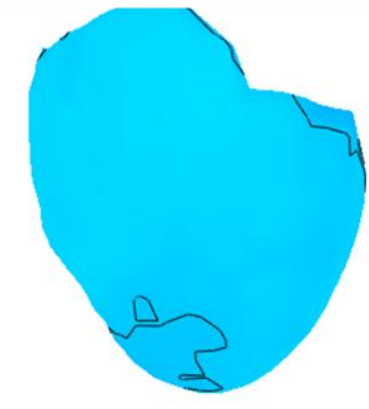

b)

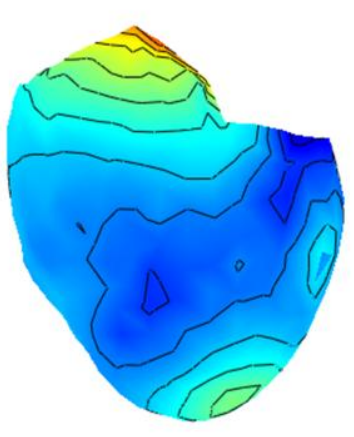

d)

Figure 4.14: Epicardial potential map $10 \mathrm{~dB}$ SNR for93 ${ }^{\text {th }}$ time instant. a) Real Epicardial Potentials, b) Tikhonov method, c) Multiple Constraints (MCC), d) Multiple Constraints (GA)

In Figure 4.14, the epicardial potentials at the $93^{\text {rd }}$ time instant are demonstrated. Here the wave front has almost propagated through all of the ventricular regions. At this time instant, multiple constraint approaches have better performance than the Tikhonov method. This fact is consistent with the CC and the RDMS values displayed in Figure 4.10 and Figure 4.11, respectively. 


\subsection{Spatio -Temporal Constraints}

In this section, we study the effects of using one spatial and one temporal constraint, as introduced in section 3.3.2. We choose the spatial constraint as the energy constraint; therefore in equation 3.11 we choose the identity matrix as the spatial regularization matrix. For the temporal regularization matrix, we used the same $\Gamma$ used in [20]:

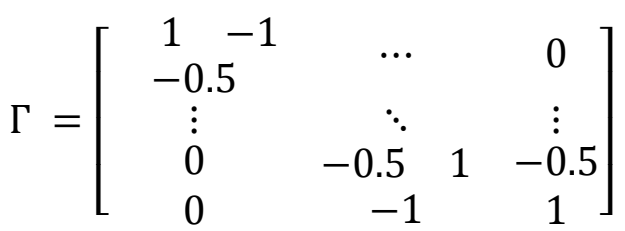

This matrix is a simple high pass filter. Again, we use the MCC and the GA approaches to calculate $\lambda$ and $\eta$, which are the spatial and the temporal regularization parameters, respectively. The results obtained by these two regularization parameter selection approaches are compared with each other and with the Tikhonov regularization with only the energy constraint. All inverse solutions are also compared with the original epicardial potential distribution, using the quantitative and the qualitative measures introduced in Section 4.2. 


\subsubsection{One Spatial and One Temporal Constraints with $30 \mathrm{~dB}$ Noise Data}

In the following section Multiple Spatial-Temporal (MST) method is applied to data with $30 \mathrm{~dB}$ SNR.

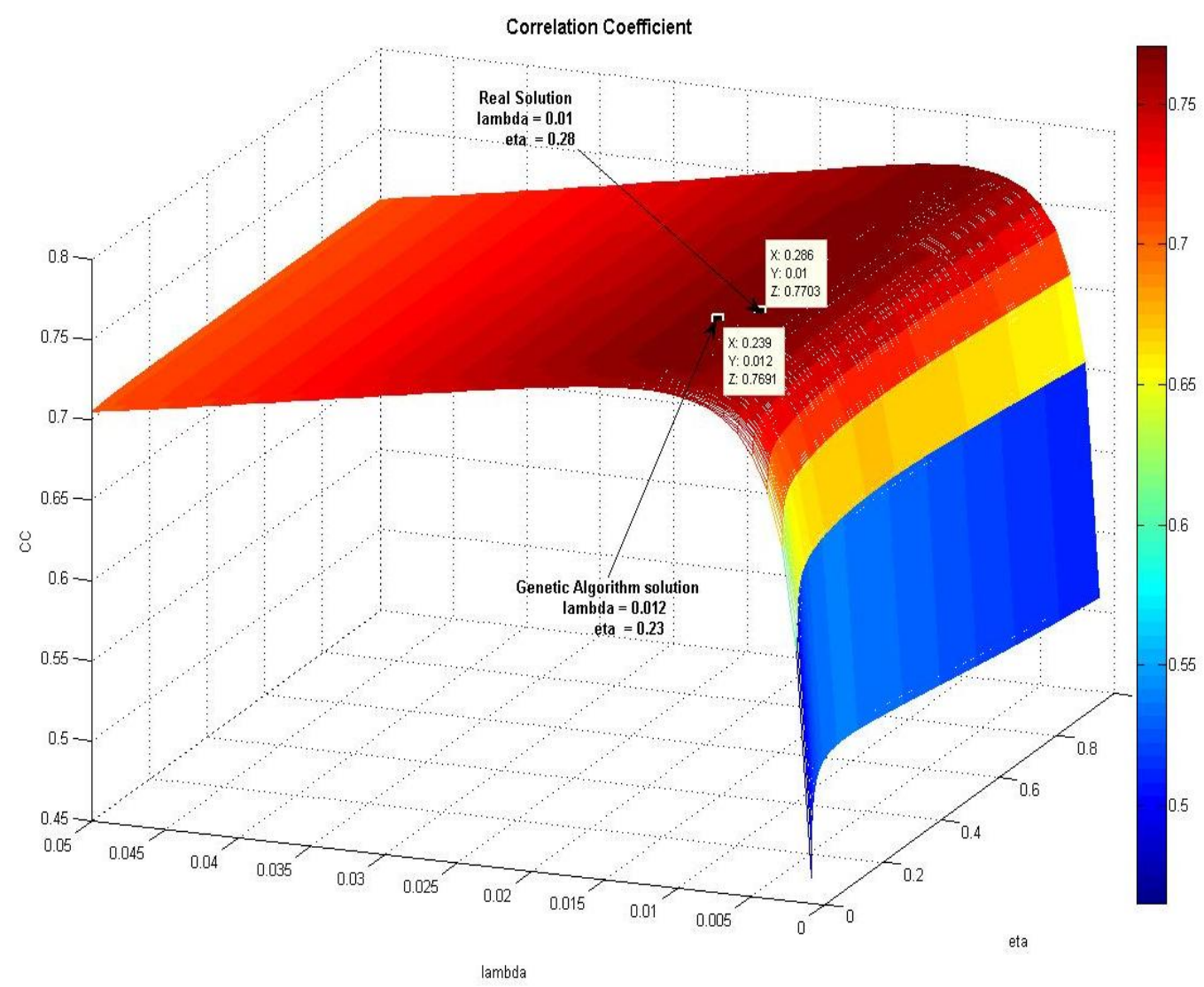

Figure 4.15: Correlation Coefficient calculated for all $\boldsymbol{\lambda}$ and $\boldsymbol{\eta}$ values using MCC method

In Figure 4.15 a 3D representation of the $\mathrm{CC}$ values corresponding to all regularization parameter pairs are plotted, and the regularization parameter choices for both MCC and GA methods are marked. As it could be seen from this figure, the regularization parameter pairs obtained by the GA are very similar those calculated by using the MCC method. The results shown in Table 4.7 approves these observations by looking at this table we can see that the CC and RDMS results for MCC and GA methods are very similar. 
Table 4.7 : MCC and GA method solution results for $30 \mathrm{~dB}$ SNR data.

\begin{tabular}{ccc}
\hline 30 dB SNR data & GA method & Maximum CC method \\
\hline CC & $0.7691 \pm 0.11$ & $0.7706 \pm 0.11$ \\
RDMS & $0.6274 \pm 0.13$ & $0.6258 \pm 0.12$ \\
$\boldsymbol{\lambda}$ value & 0.12 & 0.010 \\
$\boldsymbol{\eta}$ value & 0.23 & 0.28 \\
\hline
\end{tabular}

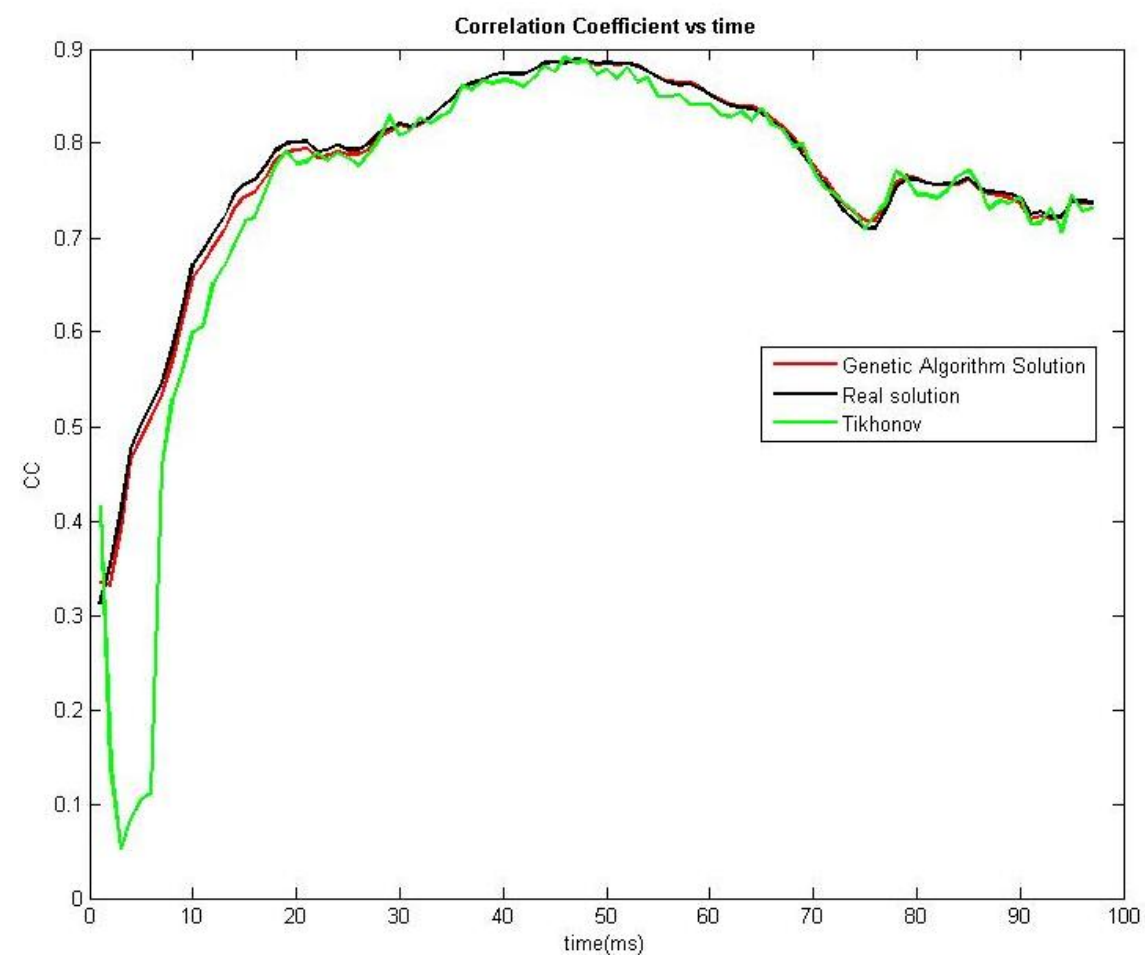

Figure 4.16 : CC figures for each time instant 


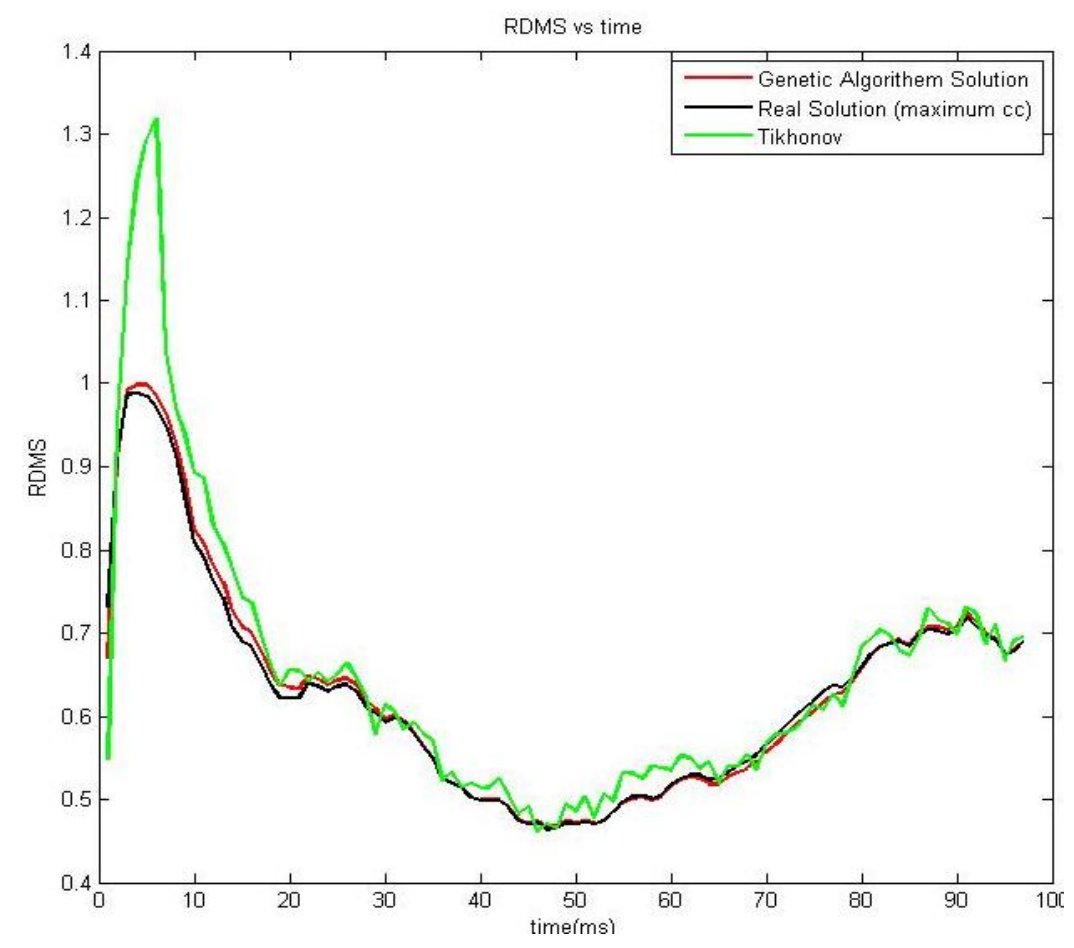

Figure 4.17: RDMS figures for each time instant.

Figure 4.16 and Figure 4.17 show CC and RDMS plots, respectively, for the Tikhonov regularization and the multiple constraints method with the GA and the MCC approaches. GA method gives very similar results with the MCC method. As explained in the previous section Tikhonov solution at the earlier time frames cannot produce impressive results; at these time frames, the $\mathrm{CC}$ value drops to very small values and RDMS peaks, but at the rest of the time instants, the results produced by Tikhonov method are acceptable.

Table 4.8: Means and standard deviations of CC and RDMS values

\begin{tabular}{lll}
\hline 30 dB SNR data & CC & RDMS \\
\hline Tikhonov & $0.74 \pm 0.17$ & $0.65 \pm 0.17$ \\
$\begin{array}{l}\text { Multiple Constraints (genetic } \\
\text { algorithm) }\end{array}$ & $0.76 \pm 0.11$ & $0.62 \pm 0.13$ \\
$\begin{array}{l}\text { Multiple Constraints } \\
\text { (maximum cc) }\end{array}$ & $0.77 \pm 0.11$ & $0.62 \pm 0.12$ \\
\hline
\end{tabular}


Table 4.9: The average and standard deviation values of RDMS and CC of the second data set.

\begin{tabular}{lll}
\hline 30 dB SNR data & CC & RDMS \\
\hline Tikhonov & $0.76 \pm 0.12$ & $0.66 \pm 0.15$ \\
$\begin{array}{l}\text { Multiple Constraints (genetic } \\
\text { algorithm) }\end{array}$ & $0.78 \pm 0.07$ & $0.63 \pm 0.10$ \\
$\begin{array}{l}\text { Multiple } \\
\text { Constraints (maximum cc) }\end{array}$ & $0.78 \pm 0.06$ & $0.63 \pm 0.10$ \\
\hline
\end{tabular}

By looking at the Figure 4.16 and Figure 4.17 show CC and RDMS plots, respectively, for the Tikhonov regularization and the multiple constraints method with the GA and the MCC approaches. GA method gives very similar results with the MCC method. As explained in the previous section Tikhonov solution at the earlier time frames cannot produce impressive results; at these time frames, the CC value drops to very small values and RDMS peaks, but at the rest of the time instants, the results produced by Tikhonov method are acceptable.

Table 4.8 and Table 4.9, we can see that the multiple constraints method with the regularization parameters chosen by the GA method performs slightly better than the Tikhonov regularization method. 


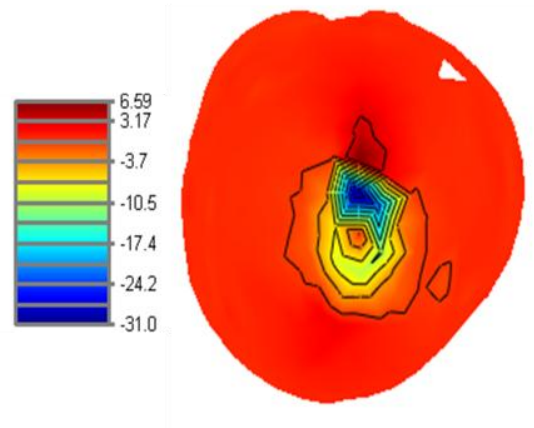

a)

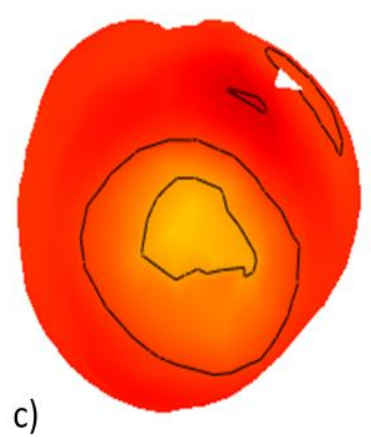

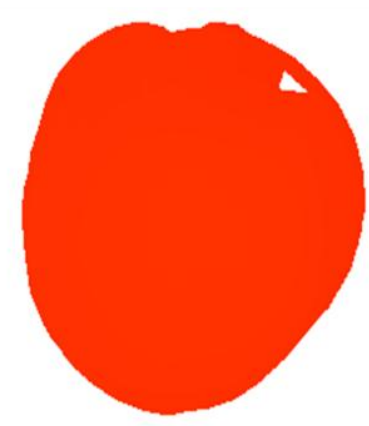

b)

d)

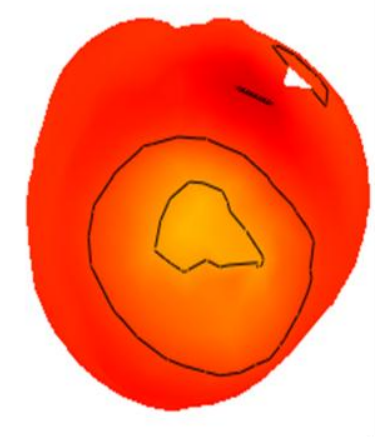

Figure 4.18 epicardial potentials 6th time instant. a) Real Epicardial Potentials, b) Tikhonov method, c) Multiple Constraints (MCC), d) Multiple Constraints (GA).

In Figure 4.18 epicardial potentials at the $6^{\text {th }}$ time instant are demonstrated. The initial activation point seen in the real epicardial potential distribution cannot be observed in the Tikhonov solution. In the maps with the multiple constraints method using both MCC and GA approaches the earliest activated region is visible; even though it is smoothed. This comparison of the three methods is also supported by the plots in Figure 4.16 and Figure 4.17. 


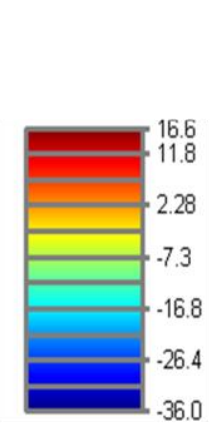

a)

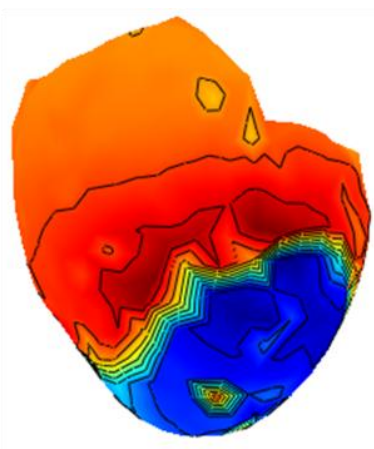

c)

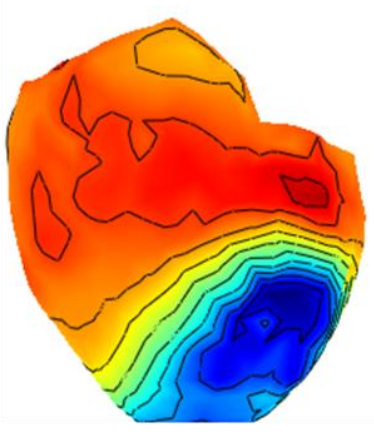

b)
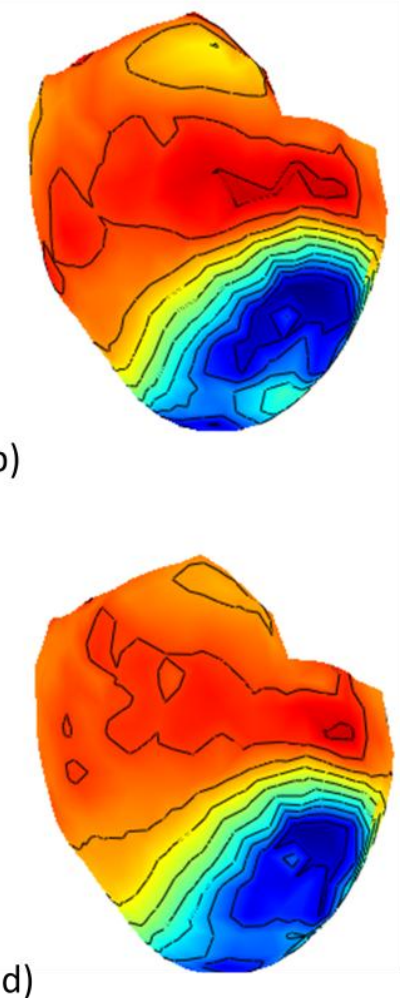

Figure 4.19 epicardial potential map for 47 time instant. a) Real Epicardial Potentials, b) Tikhonov method, c) Multiple Constraints (MCC), d) Multiple Constraints (GA).

In the rest of the time instants, all the methods reconstruct the epicardial potentials almost the similarly. For example, Figure 4.19 demonstrating the epicardial maps at the $47^{\text {th }}$ time instant, shows that the reconstructed epicardial potentials with all the methods do not have any distinguishing differences. 


\subsubsection{One Spatial One Temporal Constraints with $10 \mathrm{~dB}$ Noise Data}

In this section, we present results with the data simulated at $10 \mathrm{~dB}$ SNR.

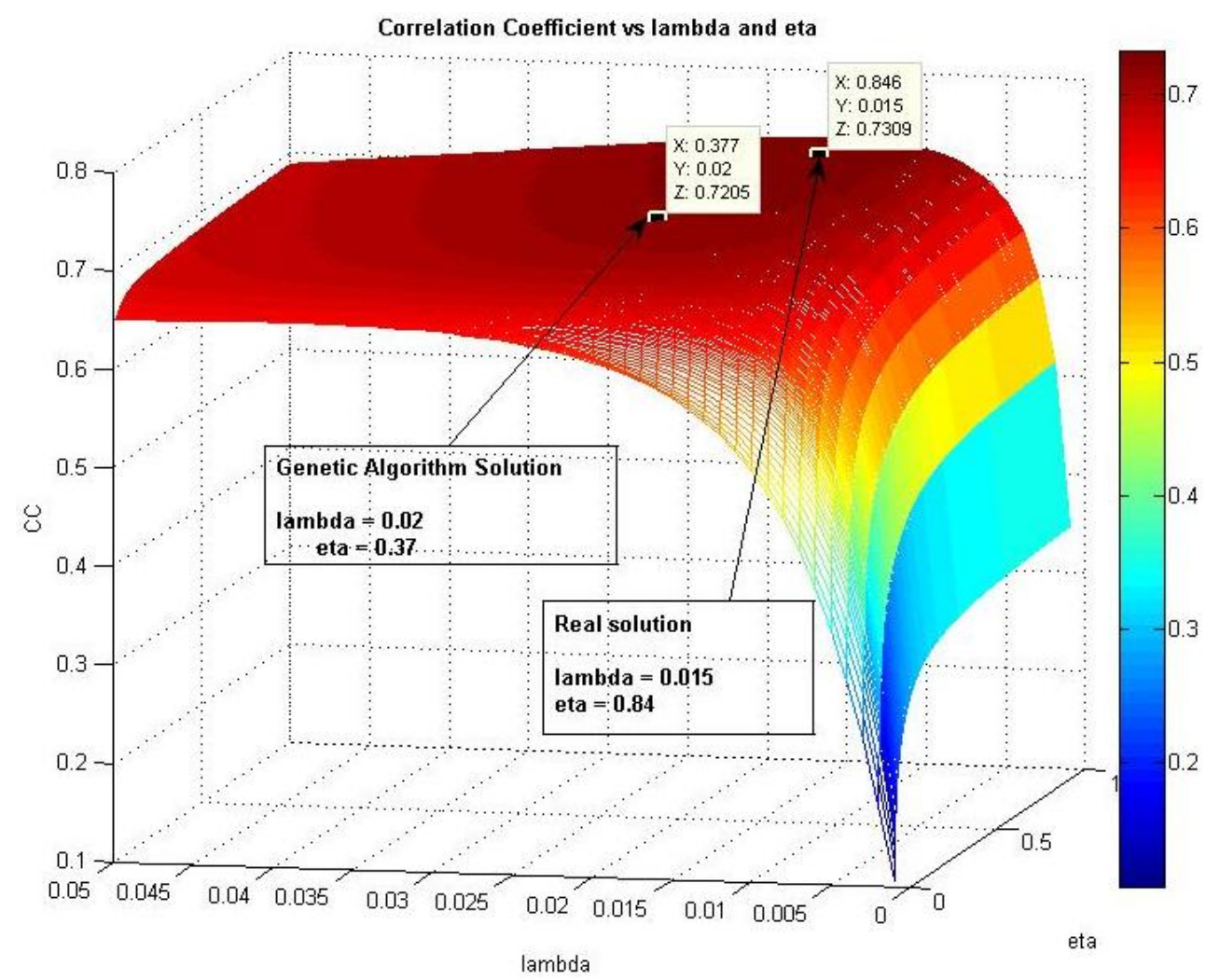

Figure 4.20 Correlation coefficient calculated for all $\boldsymbol{\lambda}$ and $\boldsymbol{\eta}$ values using MCC method for data with $10 \mathrm{~dB}$ SNR.

In Figure 4.20, a 3D representation of the $\mathrm{CC}$ values corresponding to all regularization parameter pairs are plotted, and the regularization parameter choices for both MCC and GA methods are marked. Although we can see a difference in the temporal regularization parameter choices of each approach $\left(\eta_{M C C}-\eta_{G A}=0.469\right)$, the $\mathrm{CC}$ values corresponding to both pair of parameters are close. We can see from this figure that the spatial regularization parameter is more dominant and the change in the $\mathrm{CC}$ value along the $\eta$ direction is less dramatic. This similarity of the 
performances of the two approaches is also apparent in the CC and RDMS values given in Table 4.10.

Table 4.10: MCC and GA method solution results for $10 \mathrm{~dB}$ SNR data.

\begin{tabular}{ccc}
\hline 10 dB SNR data & GA method & Maximum CC method \\
\hline CC & $0.7205 \pm 0.19$ & $0.7309 \pm 0.16$ \\
RDMS & $0.6976 \pm 0.21$ & $0.6733 \pm 0.18$ \\
$\boldsymbol{\lambda}$ value & 0.02 & 0.015 \\
$\boldsymbol{\eta}$ value & 0.37 & 0.84 \\
\hline
\end{tabular}

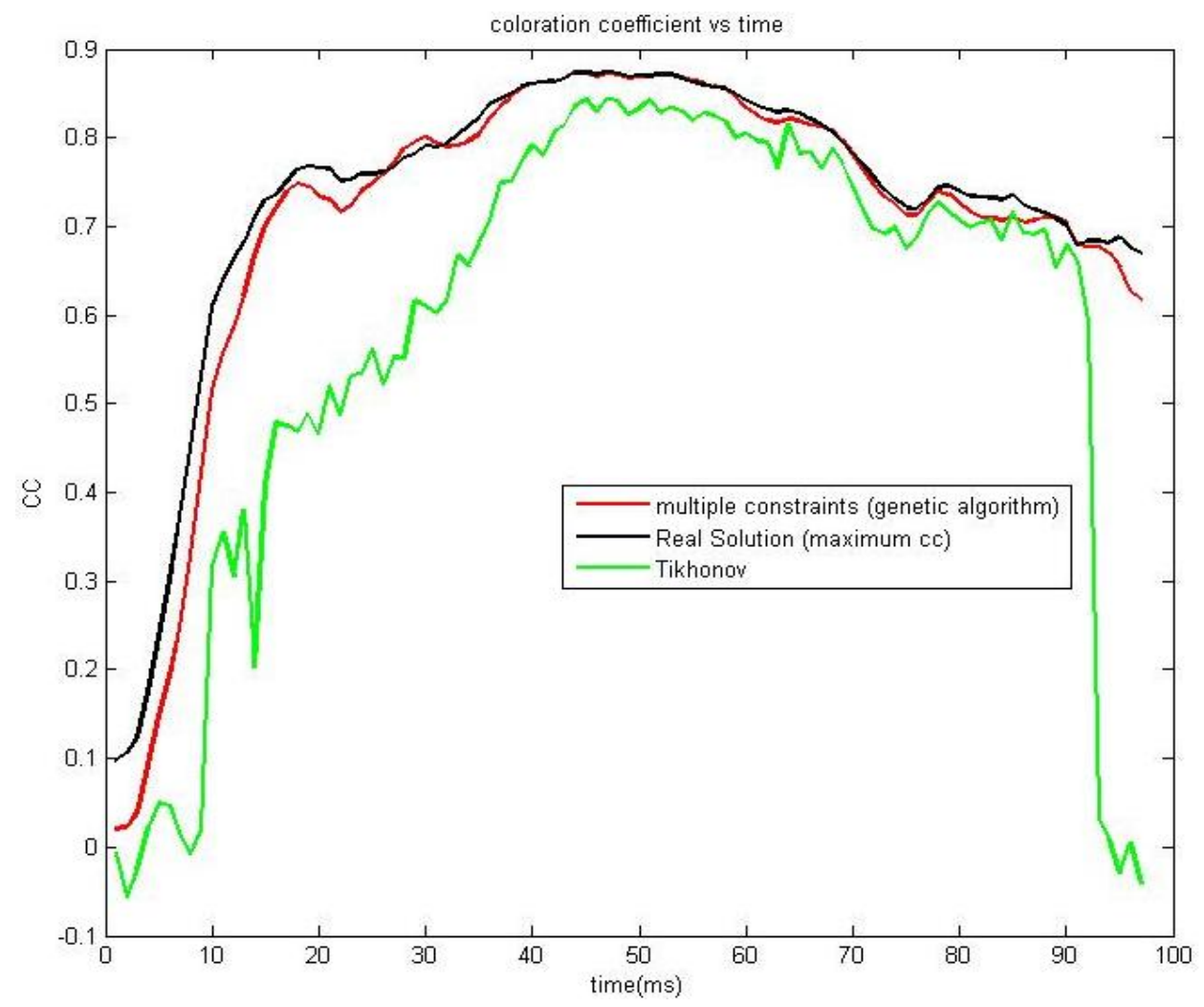

Figure 4.21 Correlation Coefficient figures for $10 \mathrm{~dB}$ SNR data. 


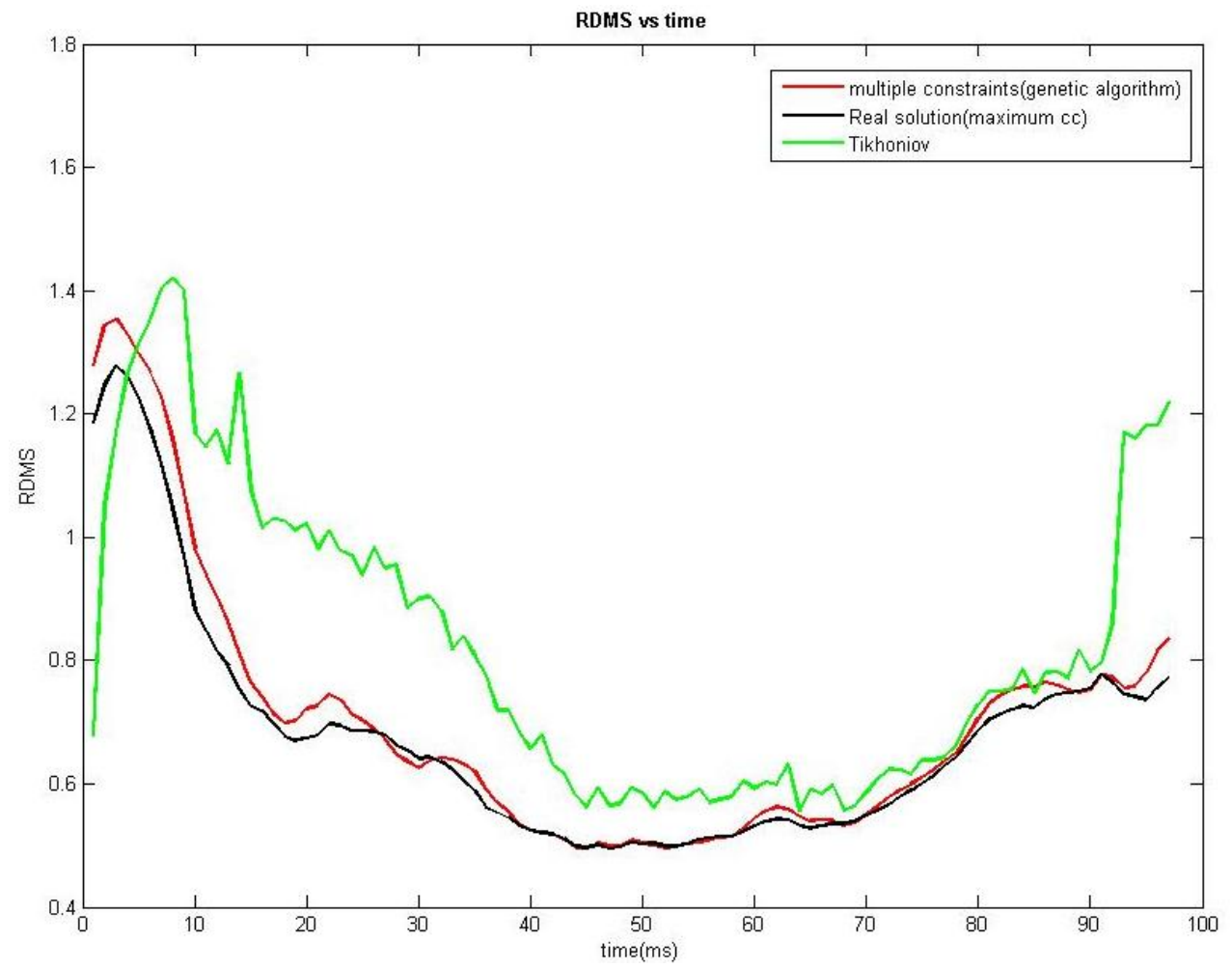

Figure 4.22 : RDMS Figures for $10 \mathrm{~dB}$ SNR data

By examining figures Figure 4.21 and Figure 4.22 and Table 4.11, we can see that by using multiple spatial-temporal approaches, we have a significant improvement in the $\mathrm{CC}$ and the RDMS values compared to the Tikhonov regularization solution.

Table 4.11 : The average and standard deviation values of RDMS and CC

\begin{tabular}{lcc}
\hline 10 dB SNR data & CC & RDMS \\
\hline Tikhonov & $0.58 \pm 0.27$ & $0.82 \pm 0.24$ \\
$\begin{array}{l}\text { Multiple Constraints (genetic } \\
\text { algorithm) }\end{array}$ & $0.72 \pm 0.19$ & $0.69 \pm 0.21$ \\
$\begin{array}{l}\text { Multiple } \\
\text { Constraints (maximum cc) }\end{array}$ & $0.73 \pm 0.16$ & $0.67 \pm 0.18$ \\
\hline
\end{tabular}


Table 4.12: The average and standard deviation values of RDMS and CC of the second data set.

\begin{tabular}{lll}
\hline 10 dB SNR data & CC & RDMS \\
\hline Tikhonov & $0.62 \pm 0.24$ & $0.81 \pm 0.27$ \\
\hline $\begin{array}{l}\text { Multiple Constraints (genetic } \\
\text { algorithm) }\end{array}$ & $0.74 \pm 0.14$ & $0.66 \pm 0.16$ \\
\hline $\begin{array}{l}\text { Multiple Constraints } \\
\text { (maximum cc) }\end{array}$ & $0.75 \pm 0.13$ & $0.66 \pm 0.15$ \\
\hline
\end{tabular}

Comparing the average CC and RDMS values for Tikhonov regularization in Table 4.8 and Table 4.11, we observe that performance of this approach drops when SNR value is lower; hence this method is highly sensitive to noise. Nevertheless, the choice of the regularization parameter plays a great role in the performance of Tikhonov method, and L-curve cannot provide the best parameter for lower SNR case; if the regularization parameter, $\lambda$, used with the Tikhonov regularization is set to the spatial regularization parameter that was calculated by the MST approach, the CC value in Table 4.11 would increase to $0.64 \pm 0.21$ and the RDMS value would decrease to $0.78 \pm 0.23$ for Tikhonov regularization. However, these improvements in the average CC and RDMS values still does not change the fact that multiple spatial-temporal constraint method performs better than the Tikhonov regularization. 


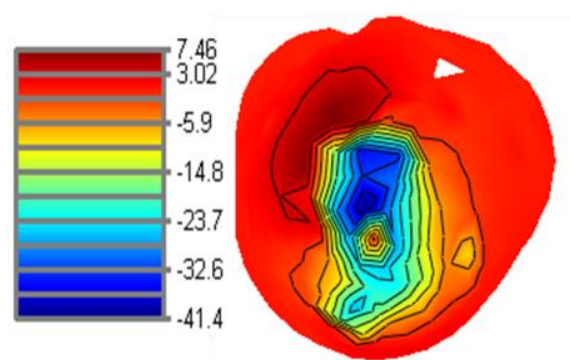

a)

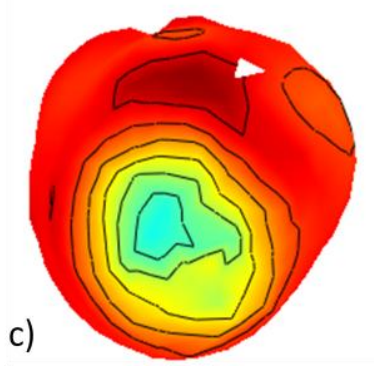

b)

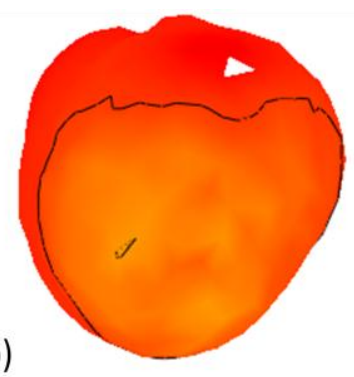

d)

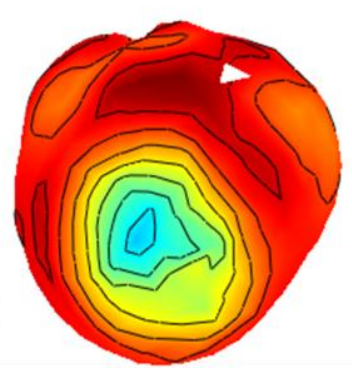

Figure 4.23 : Epicardial potentials map for 25th time instant. a) Real Epicardial Potentials, b) Tikhonov method, c) Multiple Constraints (MCC), d) Multiple Constraints (GA).

In Figure 4.23, the epicardial potential maps at the $25^{\text {th }}$ time instant are demonstrated. As it could be seen from the original epicardial potential map, an initial activation region is observed and a wave front has started to propagate on the heart surface. The epicardial potential distribution reconstructed by the Tikhonov method failed to detect this activity. On the other hand, the multiple constraints reconstructed maps, both MCC and GA approaches, have detected the initial activation region and the propagating wave front. The problem with these two approaches is that the maps are smoothed. 

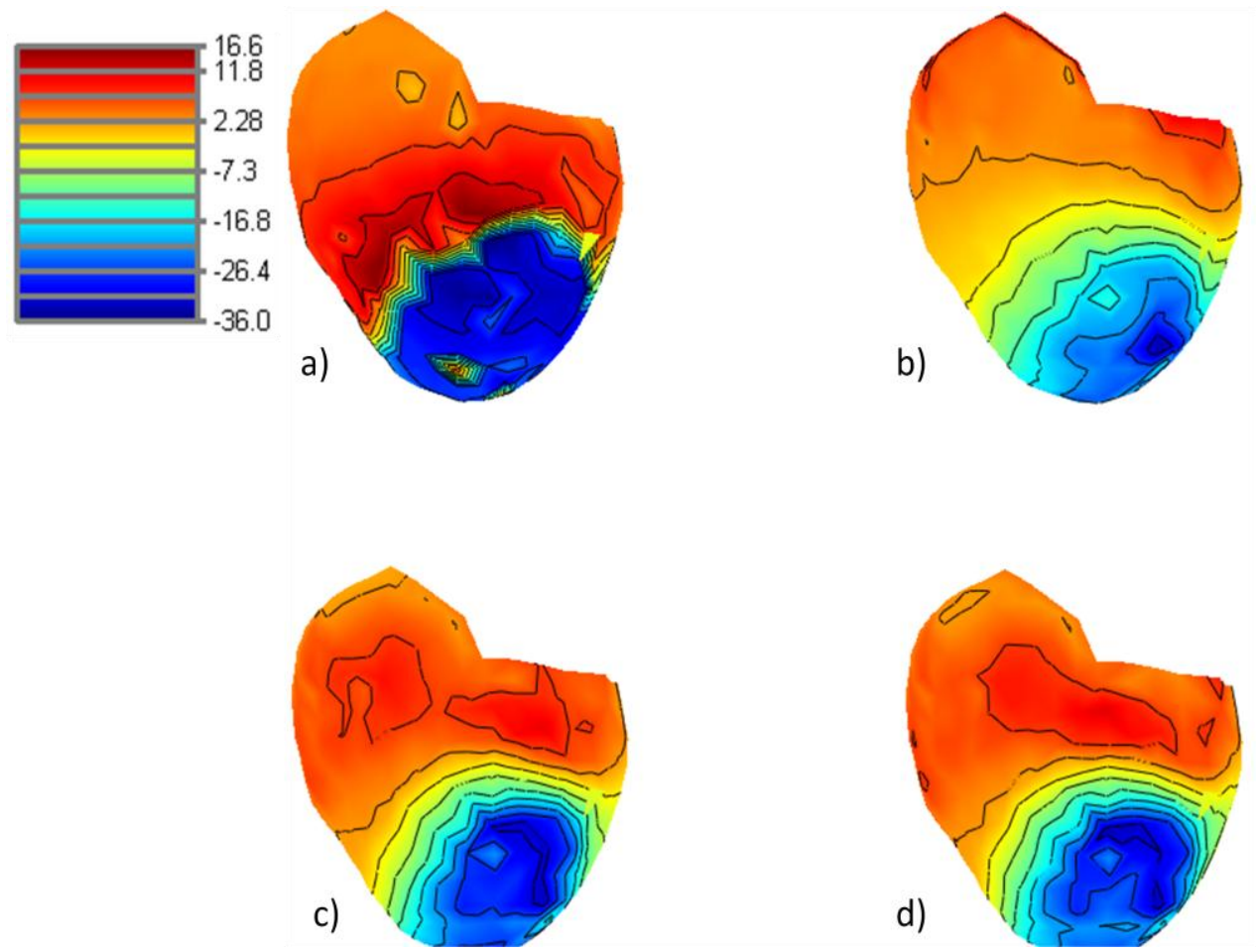

Figure 4.24 : Epicardial potentials map for 47th time instant. a) Real Epicardial Potentials, b) Tikhonov method, c) Multiple Constraints (MCC), d) Multiple Constraints (GA).

In Figure 4.24, the epicardial potential maps at the $47^{\text {th }}$ time instant are plotted. At this time instant, all of the methods reconstructed similar waveforms, but Tikhonov solution is more smoothed than the multiple constraint solutions. 

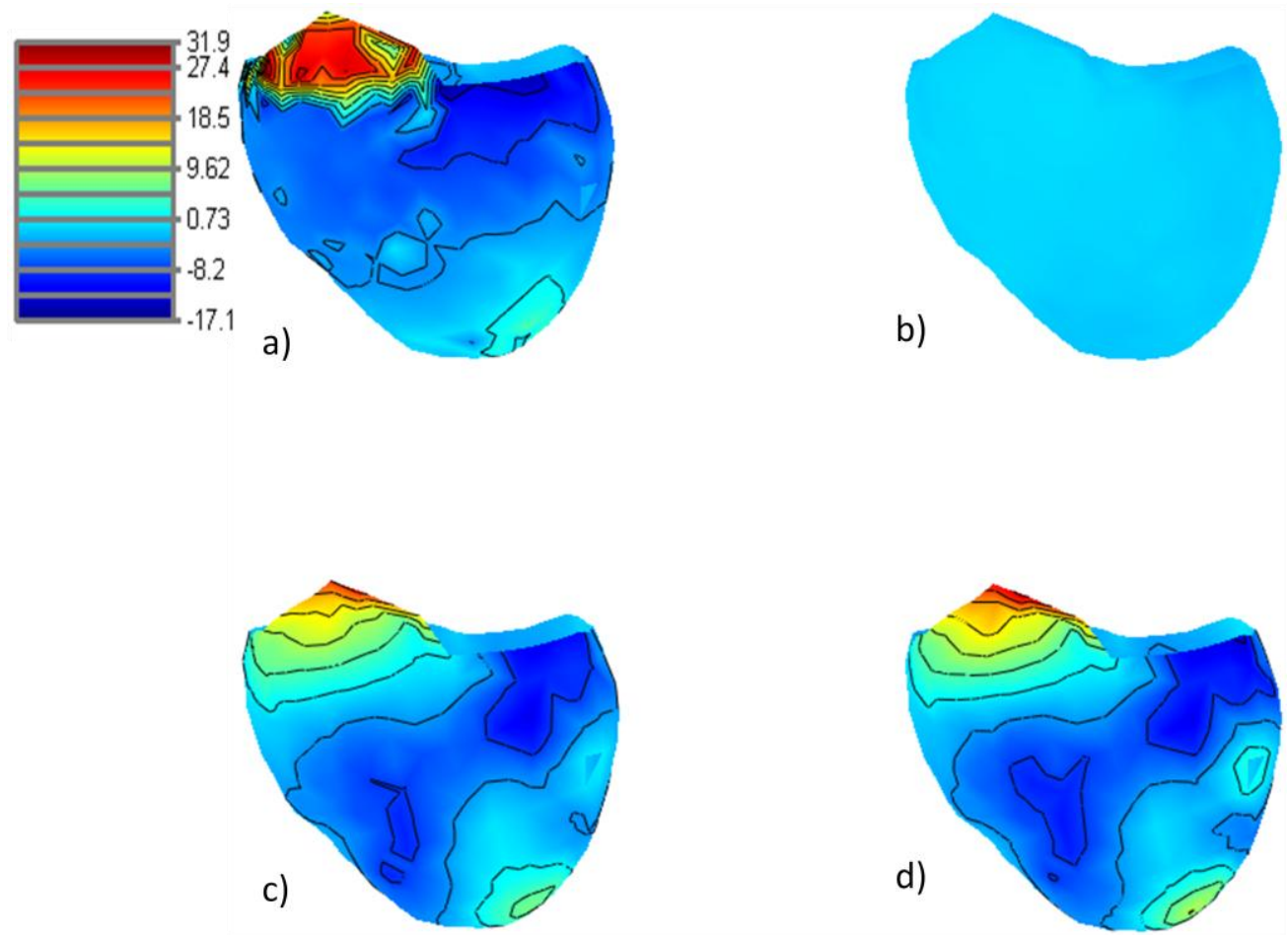

Figure 4.25 Epicardial Potential map corresponding to 93th time instant. a) Real Epicardial Potentials, b) Tikhonov method, c) Multiple Constraints (MCC), d) Multiple Constraints (GA).

In Figure 4.25, the epicardial potential maps at the $93^{\text {rd }}$ time instant are demonstrated. At this time instant, the wave front has almost propagated through the entire ventricular region. Both multiple constraints approaches, MCC and GA, reconstruct epicardial maps more similar to the original map than the Tikhonov method. Tikhonov method cannot show the wavefront; the entire ventricular surface seems to be at a value close to zero $\mathrm{mV}$. 


\subsubsection{Two Spatial One Temporal Constraints with $30 \mathrm{~dB}$ Noise Data}

In this section, we study the effects of using MST( three constraints; two spatial and one temporal). The results will be compared to MST(two constraint) method, which preformed better than MS and Tikhonov regularization methods.

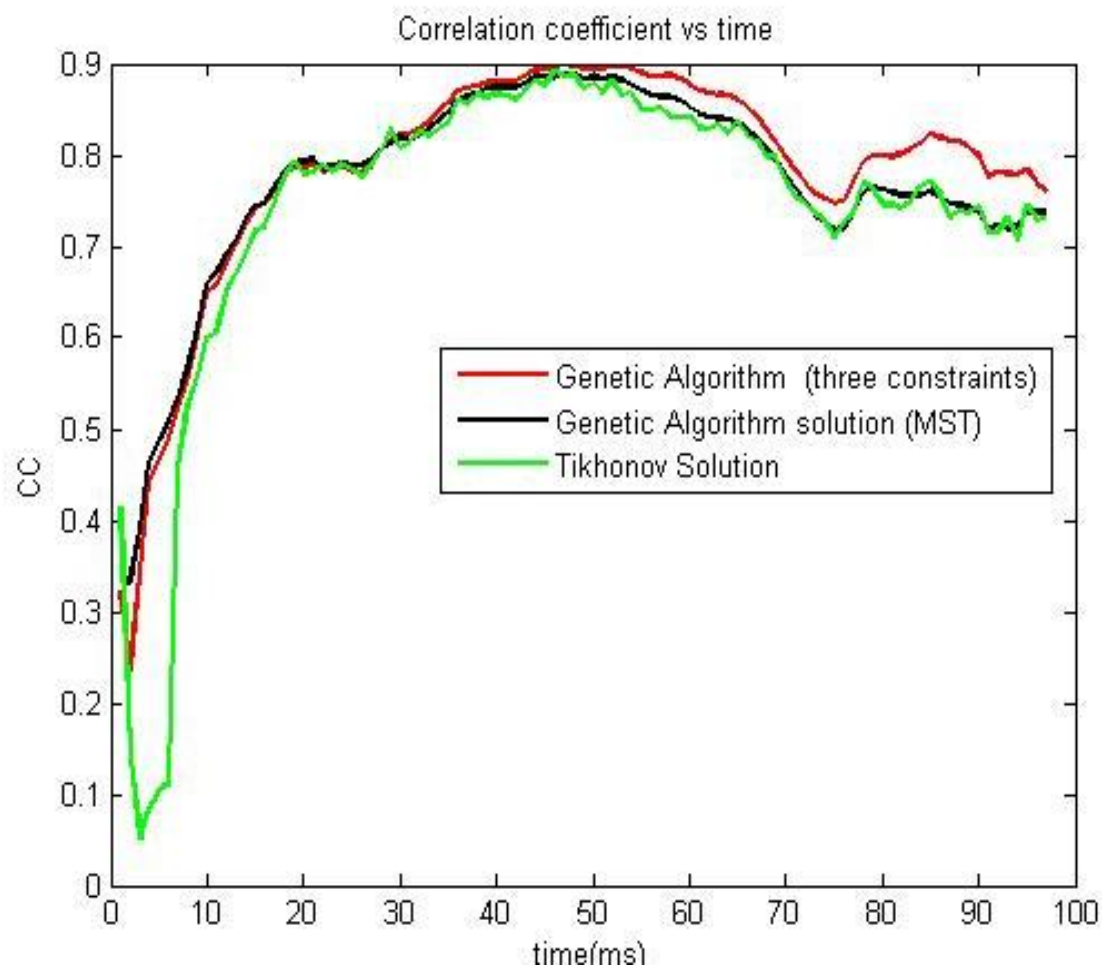

Figure 4.26 Correlation Coefficient figures for $30 \mathrm{~dB}$ SNR data. 


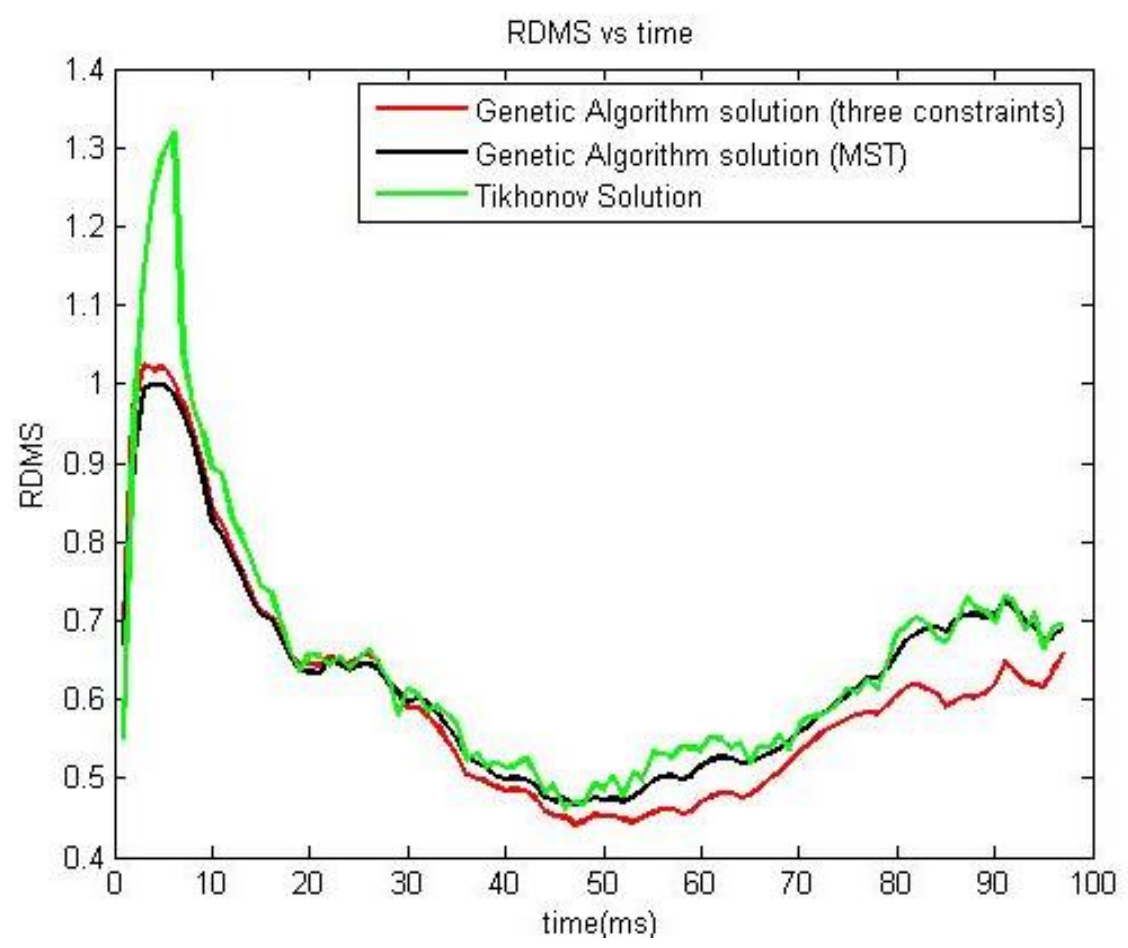

Figure 4.27 RDMS figures for $30 \mathrm{~dB}$ SNR data.

According to $\mathrm{CC}$ and RDMS plots shown in Figure 4.26 and Figure 4.27, using three constraints instead of one improves the results, especially during the second half of the QRS interval. However, this improvement is not clear when we compare the average values given in the Table 4.13 .

Table 4.13 the average and standard deviation values of RDMS and CC

\begin{tabular}{lcl}
\hline $\mathbf{3 0} \mathrm{dB}$ & CC & RDMS \\
\hline Tikhonov & $0.74 \pm 0.17$ & $0.65 \pm 0.17$ \\
Multiple Constraints (three & $0.78 \pm 0.11$ & $0.60 \pm 0.13$ \\
constraints) & & \\
Multiple Constraints (MST) & $0.76 \pm 0.12$ & $0.62 \pm 0.14$ \\
\hline
\end{tabular}




\subsubsection{Two Spatial One Temporal Constraints with $10 \mathrm{~dB}$ Noise Data}

Here, again three constraints are used to solve the problem using the proposed GA approach, however, the torso potentials are simulated at $10 \mathrm{~dB}$ SNR in this section.

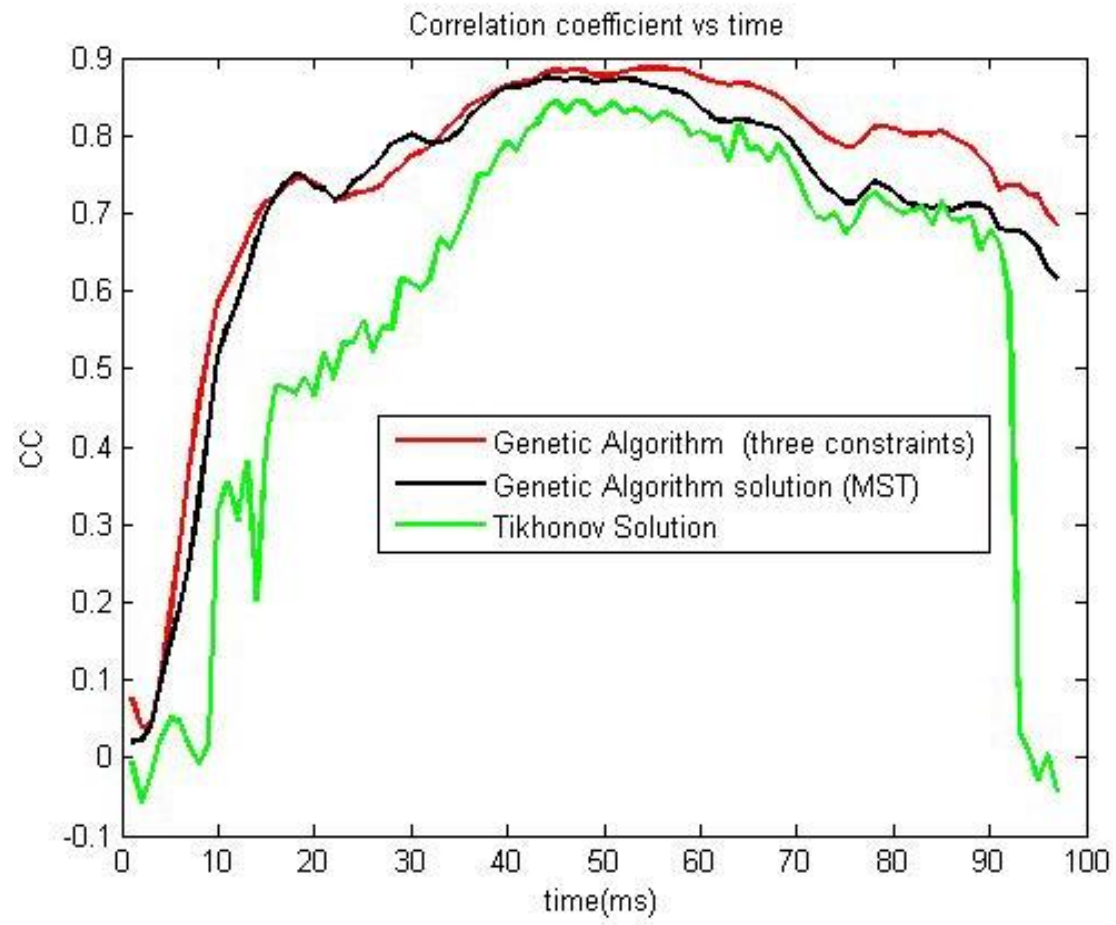

Figure 4.28 Correlation Coefficient figures for $10 \mathrm{~dB}$ SNR data. 


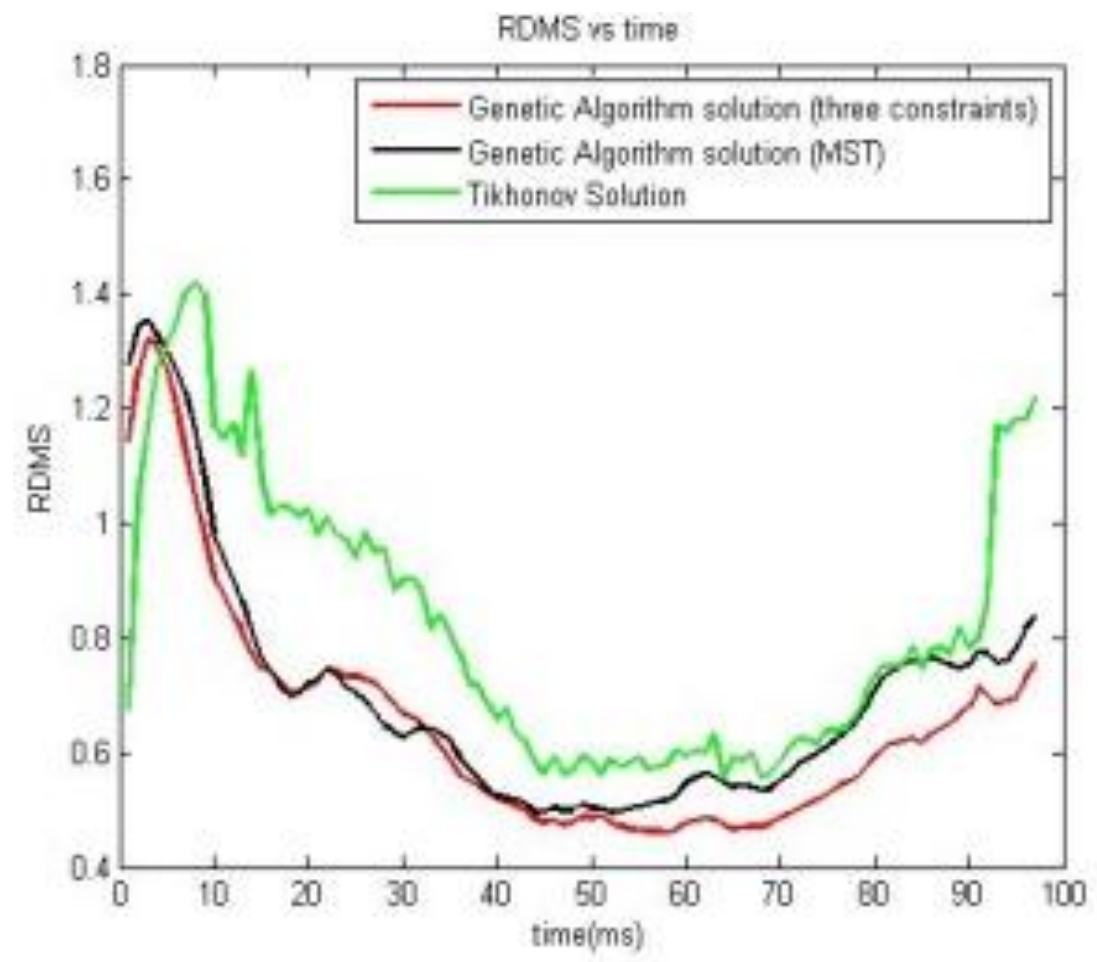

Figure 4.29 RDMS figures for $10 \mathrm{~dB}$ SNR data.

As it could be seen from the CC and the RDMS plots in Figure 4.29, Figure 4.28 using three constraints produces better results compared to Tikhonov regularization and MST methods. This fact is also reflected in the average CC and RDMS values displayed in Table 4.14.

Table 4.14 the average and standard deviation values of RDMS and CC

\begin{tabular}{lcl}
\hline $\mathbf{1 0}$ dB & CC & RDMS \\
\hline Tikhonov & $0.58 \pm 0.27$ & $0.82 \pm 0.24$ \\
Multiple constraints (three & $0.74 \pm 0.18$ & $0.65 \pm 0.20$ \\
constraints) & & \\
Multiple constraints (MST) & $0.72 \pm 0.19$ & $0.69 \pm 0.21$ \\
\hline
\end{tabular}




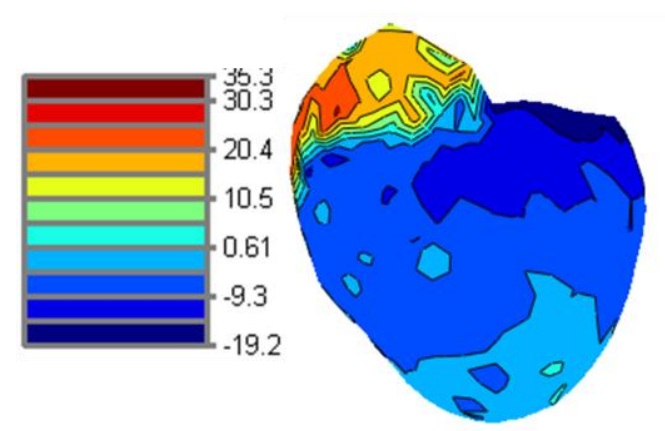

a)

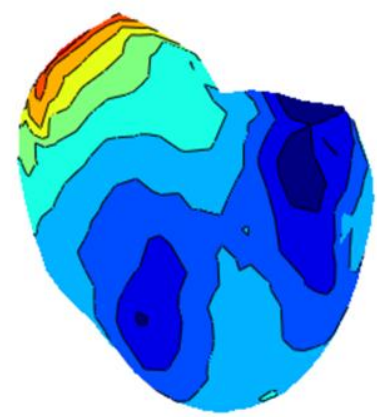

C)

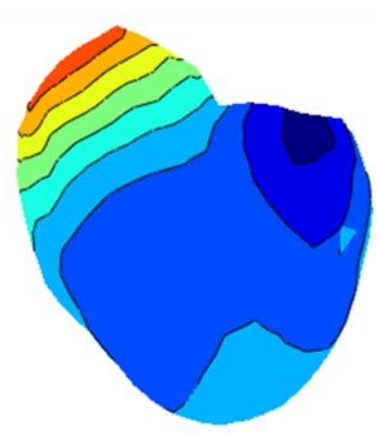

b)

d)

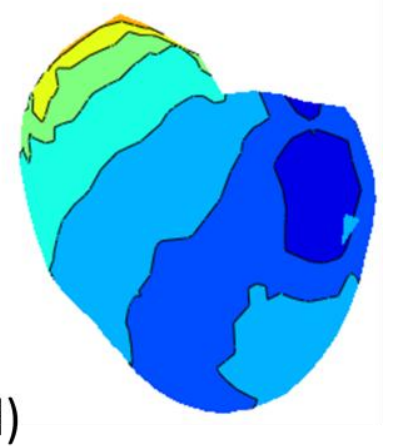

Figure 4.30 epicardial Potential maps corresponding to 85th time instant. a) Real Epicardial Potentials, b) MST (Three Constraints) c) Multiple Constraints (MST), d) Tikhonov.

Figure 4.30 plots the real and reconstructed epicardial potential map at $85^{\text {th }}$ time instant. The results obtained when three constraints are used are more similar to the real epicardial potentials at this time instant. This result is a validation of what we observed by looking at Figure 4.28 and Figure 4.29, which shows that using three constraints is better than using one or two constraints. 


\section{CHAPTER 5}

\section{INVERSE ECG TOOLBOX}

In over a decade, different methods have been introduced to solve forward and inverse problems of electrocardiography using various regularization methods. All of these methods have advantages and disadvantages which should be compared using different data sets. Thus there is a need for a tool that is easy to use to implement these methods and to compare their performances. Therefore in this study we designed a Graphical User Interface (GUI) which can be considered as an Inverse ECG Toolbox to use for this purpose. This Toolbox could also be used in biomedical engineering teaching laboratories for educational purposes.

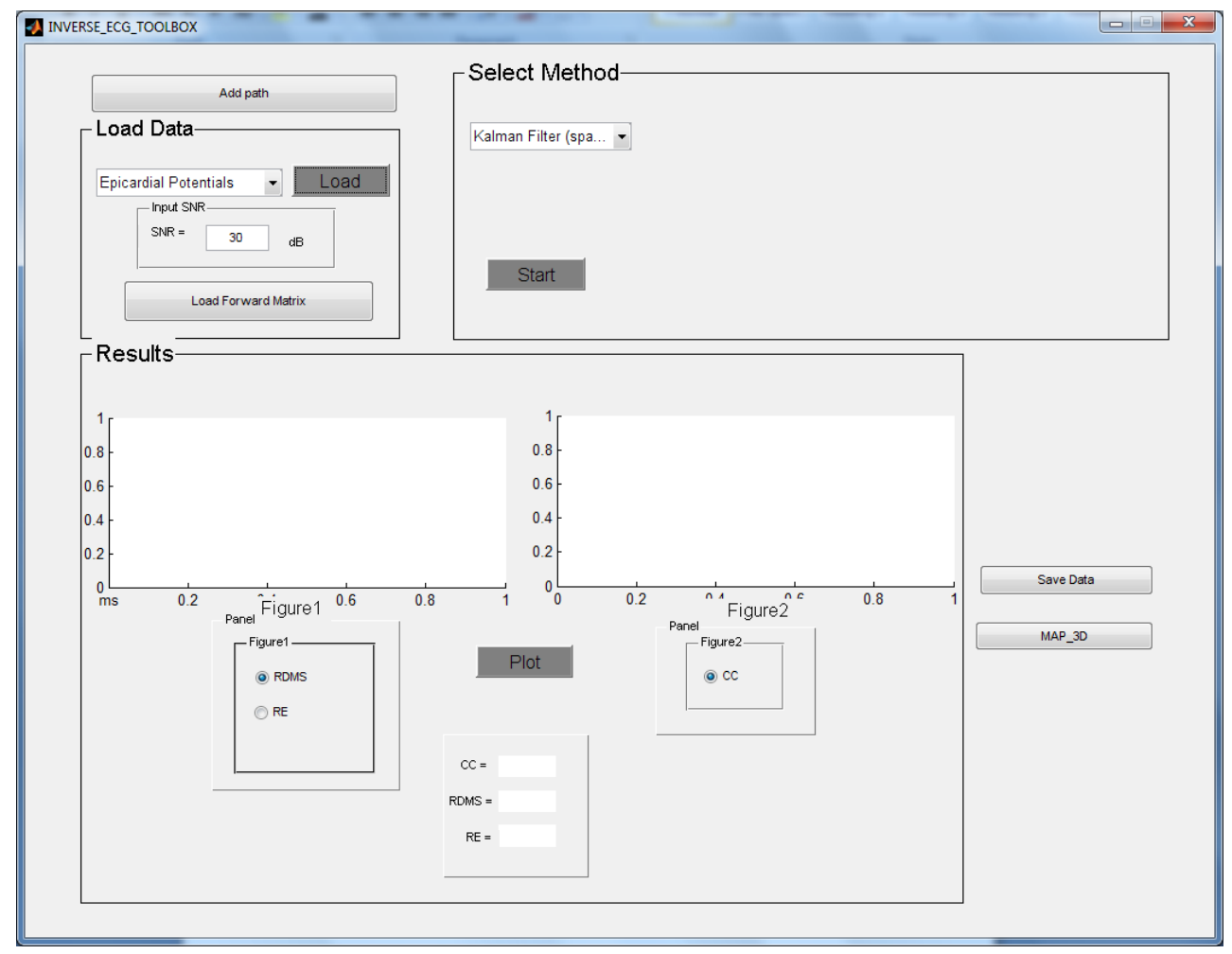

Figure 5.1 Inverse ECG Toolbox

This toolbox is composed of four major parts: 

a) Data Loading
b) Method Selection
c) Results
d) Saving data and displaying the results using Map3d.

These parts will be explained briefly in the following subsections.

\subsection{Loading Data to the Toolbox}

In this section the user loads the data that are used in all methods. In this section, the toolbox provides two options for the user:

a) Use simulated data: If the user prefers to work with s'mulated torso potentials, he or she needs to load three inputs:

1. Real epicardial potentials

2. Forward transfer matrix

3. Data SNR value

b) Use real torso data: The user can directly load measured or previously simulated "torso potentials". When this option is selected, the 2nd and 3rd inputs of case (a) above are disabled. If this option is selected only two inputs are required to be loaded.

In addition to the loaded or simulated torso potentials, the inverse solutions require a forward transfer matrix, which is also loaded by using the interface.

All the required inputs explained above are loaded automatically by the program by pressing the Load button. The corresponding Popup menu appears on the screen, the user can select the data from any folder or directory to be loaded. Figure 5.2 


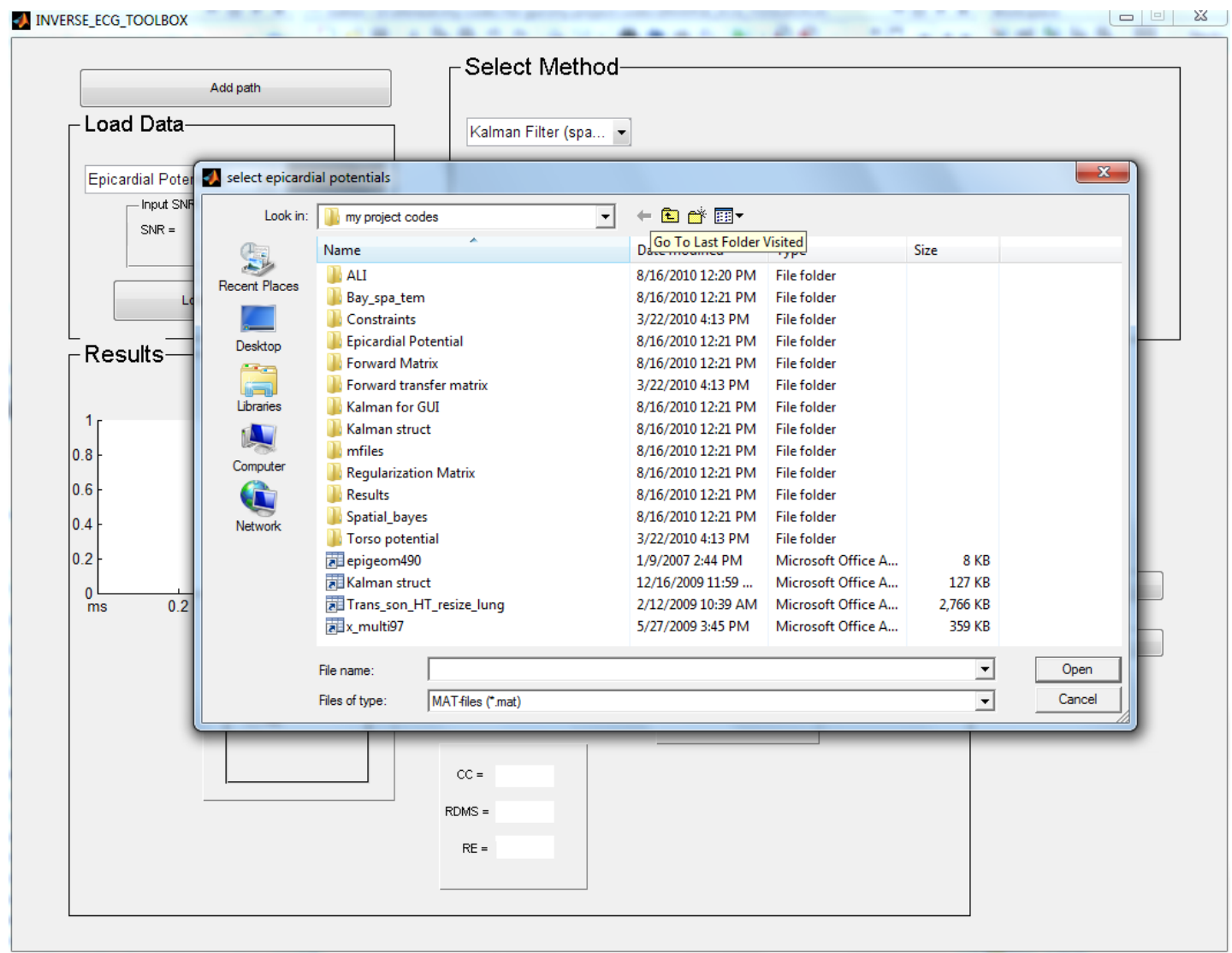

Figure 5.2 Loading data to the Toolbox.

\subsection{Selecting a Solution Method:}

In this part, the user has to choose from the methods that are listed in the select method part drop bar.

The methods listed in this menu are as follows:

1. Tikhonov (Spatial) [17]

2. Tikhonov (Spatio-Temporal) [17]

3. TSVD (Spatial) [18]

4. TSVD (Spatio-Temporal) [18]

5. Bayesian MAP (Spatial) [18][19][49][50].

6. Bayesian MAP (Spatio-Temporal) [18][19][49][50][51][52].

7. Kalman Filter [21][22][23][24][25][53][54]

8. Multiple Constraints (GA) 
When a method is selected, a panel appears on the Toolbox to input parameters needed by each method. For example Figure 5.3 shows the panel for Kalman filter.

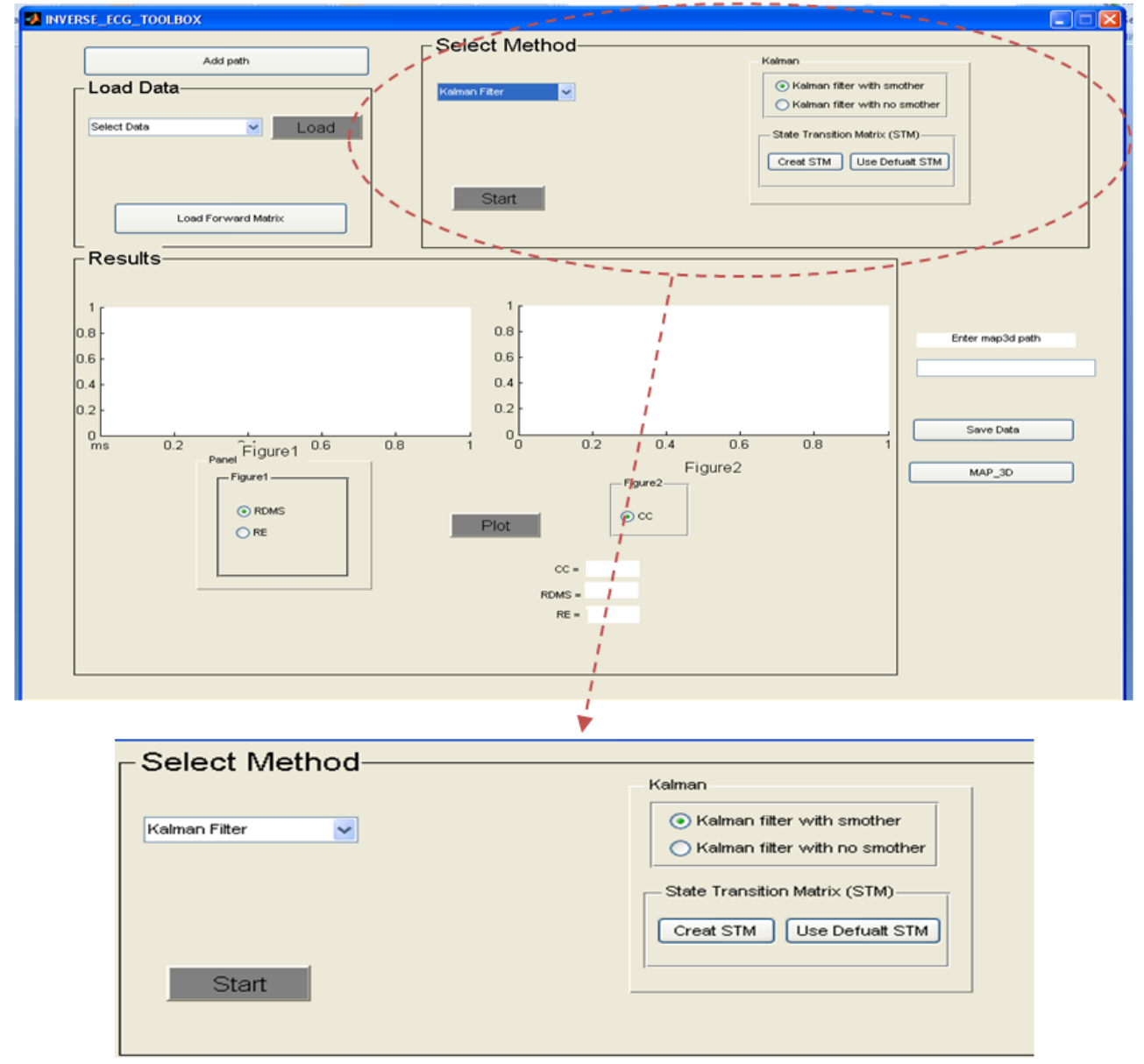

Figure 5.3: Selecting method and inputting corresponding properties.

\subsection{Display of Results:}

This part, as shown in Figure 5.4, contains two figure plots that are used to display results graphically. Correlation Coefficients (CC) versus time is plotted by default (right figure panel). Figure panel on the left offers a choice for the user; by clicking on the radio buttons under this figure panel, user can choose to plot either Relative Error (RE) versus time, or Relative Difference Measure Star (RDMS) versus time. The average values of CC, RE, and RDMS values are given in a edit box below the figure panels. 


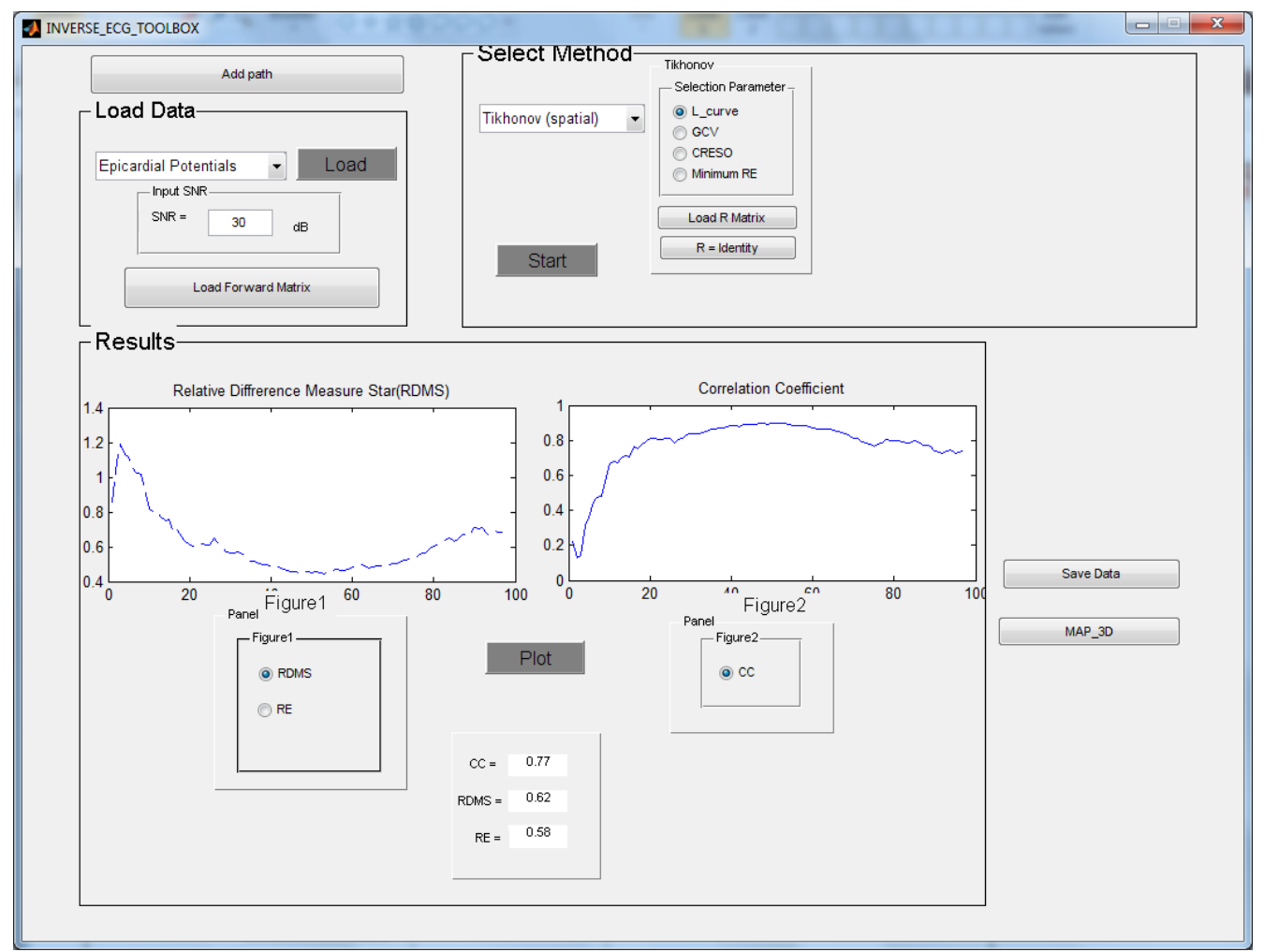

Figure 5.4: Result demonstration.

\subsection{Saving Data and MAP3D Connection:}

After running the program, the program returns the estimated epicardial potentials as a matrix. This matrix could be saved to any folder chosen by the user to be used later on for reviewing the results, or to be used with another program like MAP3D. 


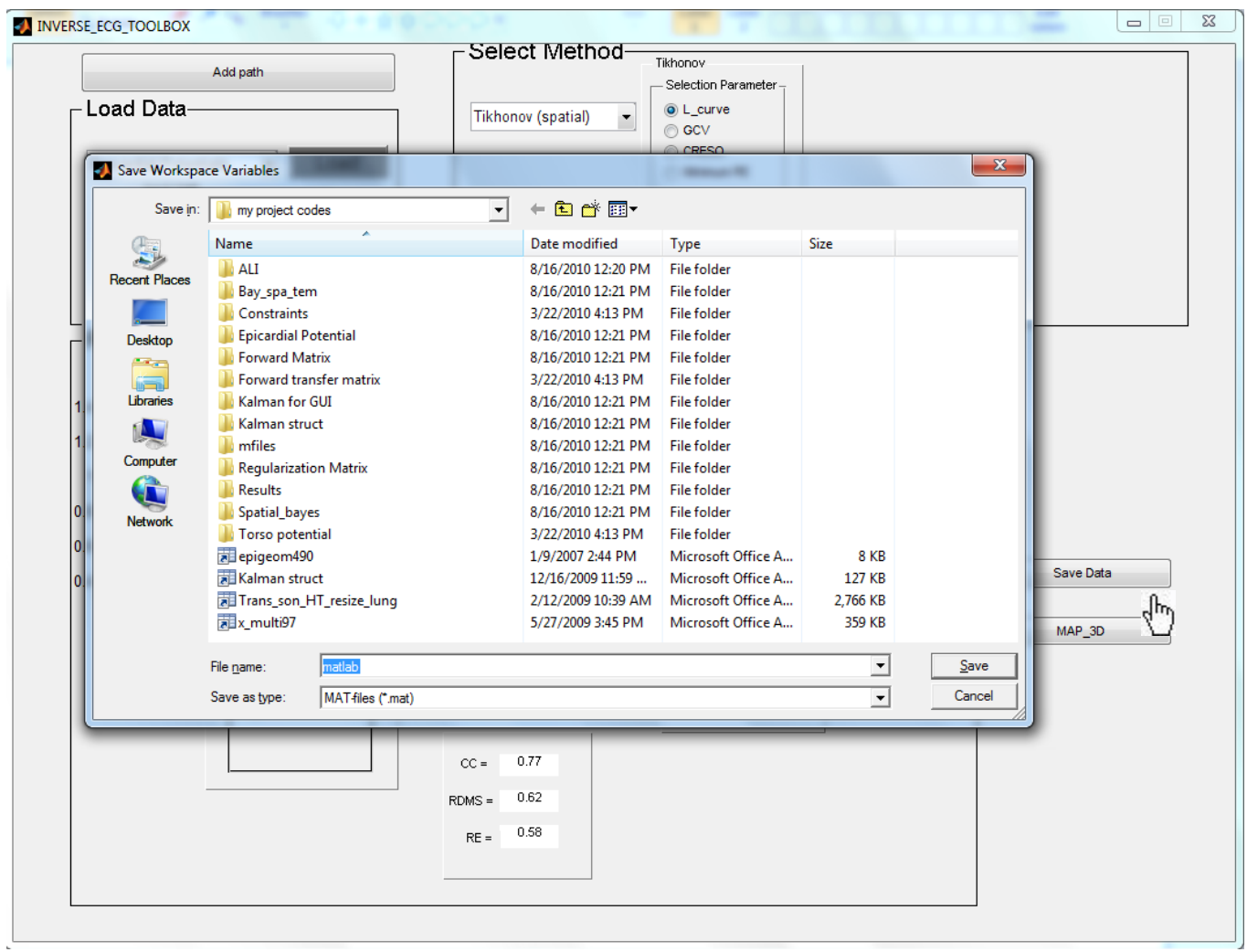

Figure 5.5: Saving Results and taking notes.

If the Save button is pressed, the user could decide where to save the obtained results. To add more information about the obtained results, a text document will be opened after the data is saved, in which the user could add any information about the obtained result, such as the SNR value of the simulated data, the method used for estimating the result, parameters used for the chosen method, etc. 


\subsubsection{MAP3d connection:}

There is a direct access to MAP3D program using the INVERSE_ECG_TOOLBOX; if the user presses the MAP3D button the MAP3D program will start running and the user could load the saved result or any result saved before to the MAP3D program.

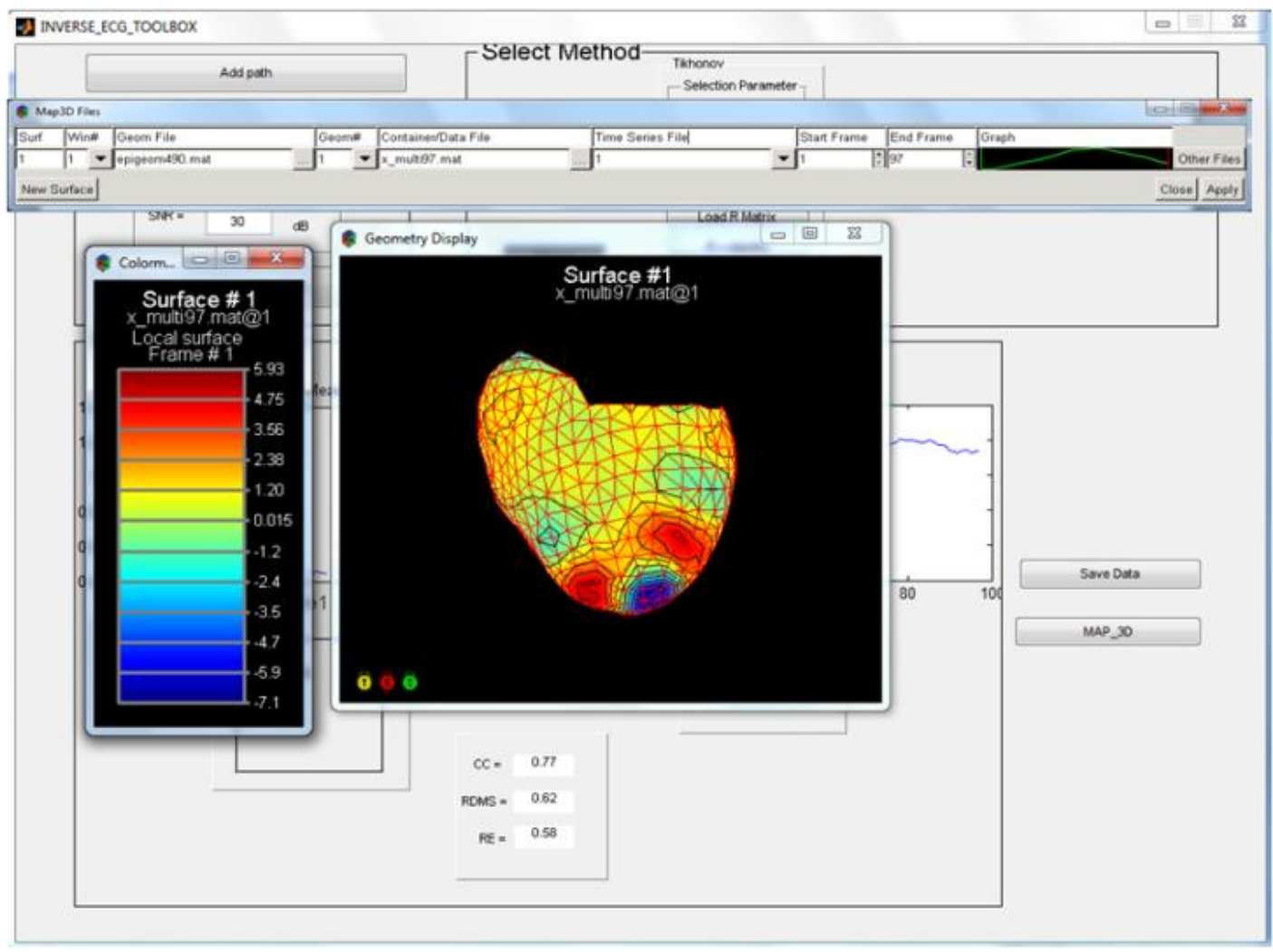

Figure 5.6: Results Display using direct connection to MAP3D 


\subsection{Toolbox Run Example}

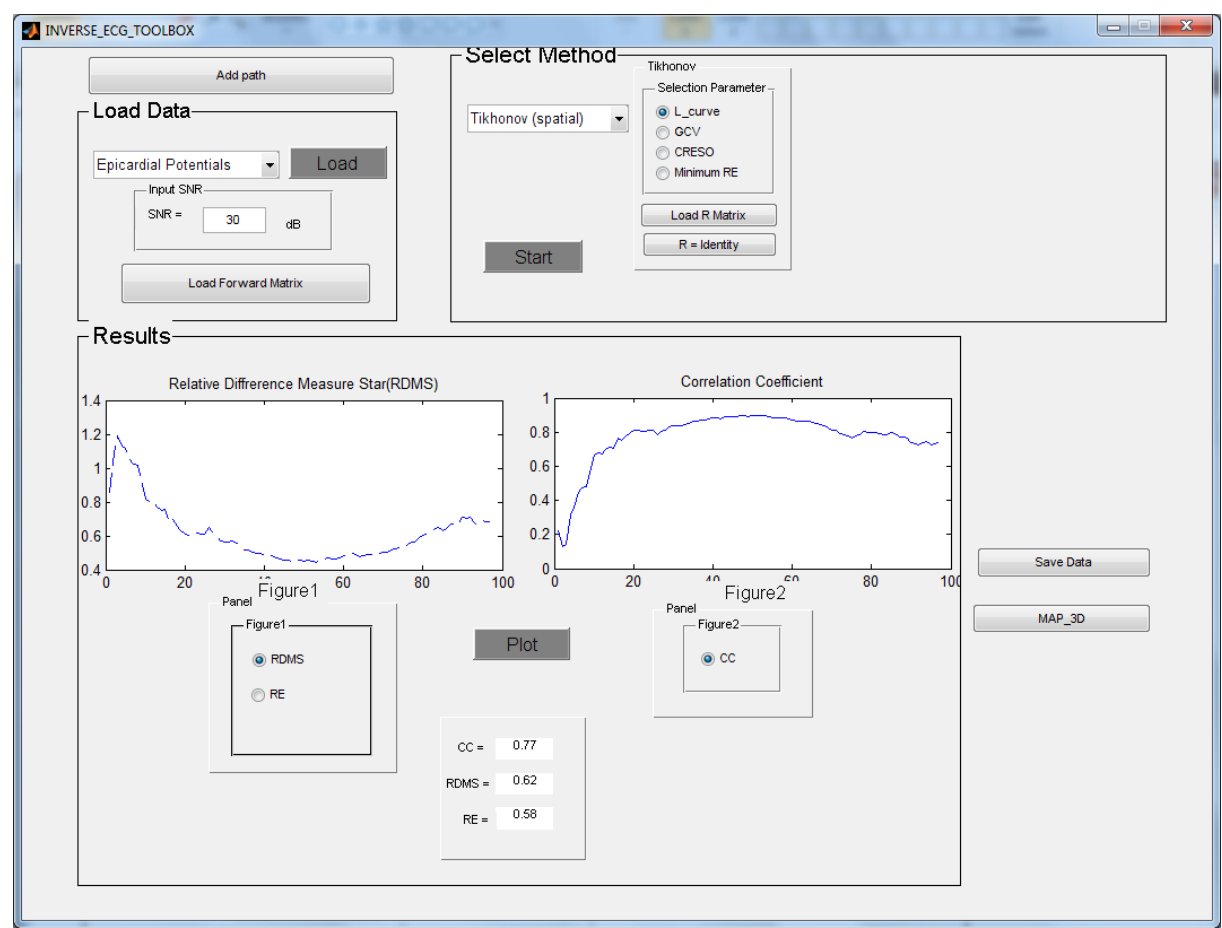

Figure 5.7 Tikhonov method.

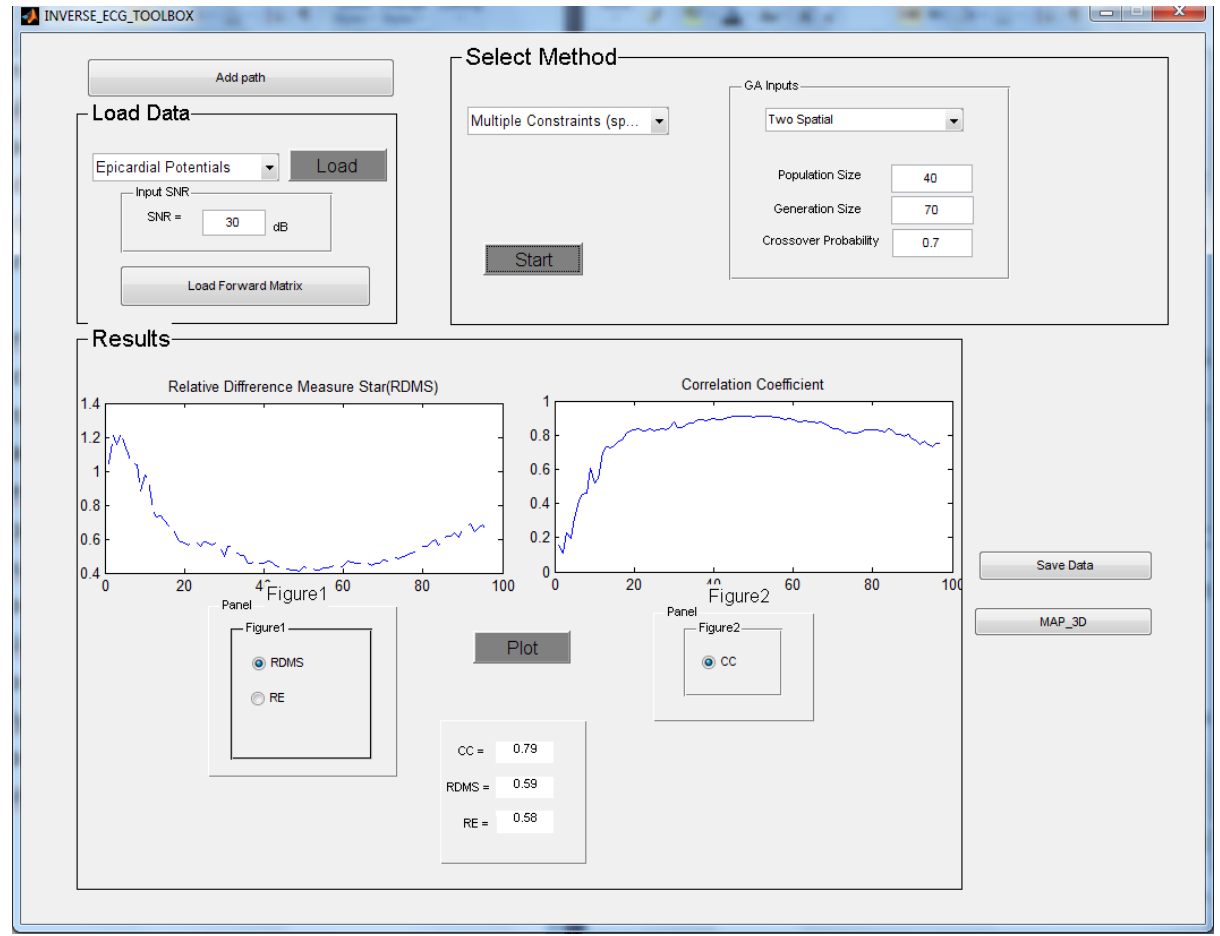

Figure 5.8 Multiple constraints method using GA 
In the Figure 5.7 and Figure 5.8 we can see a simple example of using the toolbox to compare Tikhonov regularization and multiple constraints approach using GA. Each method has a panel from which the required inputs of the corresponding method can be entered into the program. For example for multiple constraints method using GA number of populations and number of generations and the crossover probability is entered by the user and also there is an option for the user to select number of constraints that are to be used in the solution.

The average values of $\mathrm{CC}$, RDMS and RE are given for each method; also the user can compare the discussed Quantitative Comparisons methods in each time instant by using the graphs in the toolbox. 


\section{CHAPTER 6}

\section{CONCLUSION}

The main objective of this thesis is to use multiple constraints to improve the solutions discussed for solving inverse ECG problem. But the problem of finding appropriate regularization parameters restricts the application of this approach. To overcome this problem, we proposed a genetic algorithm based approach to find multiple regularization parameters. One advantage of this new approach is its adaptability to include arbitrary number of constraints. This method was not used before to solve the inverse ECG problem; thus it is a novelty in the research area.

The inferences made from our results can be summarized as follows:

- As the ground truth, we obtained the regularization parameters corresponding to two constraints using the CC values; we picked the optimum parameters so as to yield maximum $\mathrm{CC}$ values (MCC method). This is not a realistic way to obtain the regularization parameters; however it gives us an idea about what the values of "best" regularization parameters should be, and how much improvement we can obtain by the multiple constraint approach. The regularization parameters obtained by the GA algorithm are very close to the ones obtained by MCC, and the corresponding solutions are improved compared to single-constraint Tikhonov solutions.

- The regularization parameters calculated by the GA method both in Multiple Spatial (MS) and Multiple Spatial-Temporal (MST) regularization are closer to the parameters calculated by the MCC method when we simulate the measurements at $30 \mathrm{~dB}$ SNR, compared to the case with $10 \mathrm{~dB}$ SNR. Consequently, the regularization parameters obtained by the GA approach 
produce average $\mathrm{CC}$ and RDMS values very close to those obtained by MCC method. This similarity is more obvious when the SNR value is $30 \mathrm{~dB}$.

- The solutions obtained by using GA method to select the regularization parameters both in MS and MST produce results with better average CC and RDMS values compared to Tikhonov method which uses only one regularization parameter in both $30 \mathrm{~dB}$ and $10 \mathrm{~dB}$ cases. This result shows the advantage of using more than one regularization parameter.

- In the CC and RDMS plots, both multiple constraint approaches (two spatial (MS), and one temporal - one spatial constraints (MST)) produce results that can follow the MCC method, while the Tikhonov method fails to follow the MCC solution; this fact is especially more obvious in the beginning and ending regions of the QRS interval; the performance of Tikhonov regularization is worse than the multiple constraint approaches. The gap between Tikhonov regularization and the multiple constraint solutions is even larger when 10dB SNR data is used.

- Examining the MAP3D figures, we can see that Tikhonov regularization cannot reconstruct the epicardial potential distribution at the initial activation point in the first time samples, especially at $10 \mathrm{~dB}$ SNR case. Both multiple spatial, and multiple spatial-temporal constraint methods using the proposed GA approach to select the regularization parameters succeed in detecting the earliest activation regions, even for the $10 \mathrm{~dB}$ SNR data.

- By Examining the MAP3D figures we understand that all three methods (Tikhonov, MS and MST) discussed in the results chapter have smoothing effect on the solutions, although the smoothing effect of Tikhonov approach is higher than both MS and MST approaches.

- By comparing the MS and MST approaches, we can see that the MST produces better results, both in terms of the average CC and RDMS values, and in terms of visual inspection, compared to the multiple spatial constraint approach. This observation is more noticeable when SNR value is reduced. Therefore, adding a second temporal constraint is more effective than adding a second spatial constraint. 
- By comparing the MST (three constraints) and MST approaches, we can see that using three constraints produces better results, both in terms of the average $\mathrm{CC}$ and RDMS values, and in terms of visual inspection, compared to the MST constraint approach.

By examining the results obtained in using GA method to calculate the regularization parameters in a multiple constraint frame work, we can see that the GA method is very successful in calculating these parameters and due to its compatibility to be upgraded for more than two parameters depending on the problem, application of this method could improve the results and open new doors in the fields using regularization parameters in solving inverse problems.

Although both methods used in this study (MS, MST) provide an improvement in the solutions compared to using a single constraint, among the two multiple constraint approaches, MST method is preferable to MS; the computation time is comparable for both approaches, however MST yields more improved results than MS.

The maximum run time obtained when using a $2.4 \mathrm{GHz}$ processor with 6GB RAM is 600 seconds, and the average run time is 300 seconds for the same system. This is the main disadvantage of using GA method. The run time could be decreased to some extend by improvements in the programming or other programming techniques like CUDA (Compute Unified Device Architecture) programming to solve the problem permanently.

In addition to our study on determination of regularization parameters for multiple constraint inverse ECG solutions, we developed a graphical user interface to implement and compare various inverse solution approaches. This toolbox makes it easier for the user to run various solution algorithms on the same data, or with multiple data. It could also be used in biomedical engineering laboratories as an educational tool. 


\subsection{Future work}

- Practically regularizations could be used in different regions of the data for instance one regularization parameter for wave front region another for activated regions and another for inactivated points therefore multiple temporal and spatial regularization parameters could be suggested which the GA approach could be adapted to this problem.

- Studies on how to remove the smoothing effect on the multiple constraint method could improve the results obtained.

- Studies on using constraints that could improve the results.

- The forward problem of ECG could be added to the Inverse ECG Toolbox. 


\section{REFERENCES}

[1] Eriksson H. Heart failure: a growing public health problem. J Intern Med, 237:135-141, 1995.

[2] Pullan A. J., Paterson D., and Greensite F. Noninvasive imaging of cardiac electrophysiology. Phil. Trans. R. Soc. Lond. A, 359:1277-1286, 2001.

[3] Malmivuo J. and Plonsey R. Bioelectromagnetism - Principles and Applications of Bioelectric and Biomagnetic Fields. Oxford University Press, 1995

[4] Guyton A. C. and Hall J. E. Textbook of Medical Physiology. Elsevier Saunders, 2006.

[5] Cardiac Action Potential (http://en.wikipedia.org/wiki/Cardiac_action_potential), $(27.09 .2010)$

[6] Despopoulos A. and Silbernagl S. Color Atlas of Physiology. Georg Thieme Verlag, 2003.

[7] Einthoven W., Fahr G. and de Waart A., "Über die Richtung und die Manifeste Grösse der Potentialschwankungen im mennschlichen Herzen und über den Einfluss der Herzlage auf die form des Elektrokardiogramms" Pflüger Arch. ges. Physiol. 150: 275-31, 1913.

[8] Cardiovascular \& Medical Sciences, The Royal Infirmary (http://www.gla.ac.uk/researchinstitutes/cms/), (27.09.2010)

[9] Despopoulos A. and Silbernagl S. Color Atlas of Physiology. Georg ThiemeVerlag, 2003. 
[10] Gulrajani R. M. The forward and inverse problems of electrocardiography. IEEE Eng Med Biol Mag., 17:84-101,122, 1998.

[11] Seger M., Fischer G., Modre R., Messnarz B., Hanser F., and Tilg B. Lead field computation for the electrocardiographic inverse problem-finite element versus boundary elements. Computer Methods and Programs in Biomedicine, 77:241-252, 2005 .

[12] Shou C., Xia L., Jiang M., Liu F., and Crozier S. Forward and inverse solutions of electrocardiography problem using an adaptive bem method. Springer-Verlag Berlin Heidelberg FIMH, pages 290-299, 2007.

[13] Johnson C. R. and MacLeod R. S. Nonuniform spatial mesh adaptation using a posteriori error estimates: applications to forward and inverse problems. Applied Numerical Mathematics, 14:311-326, 1994.

[14] Inverse ECG (http://matthijs.cluitmans.net/academic/documents/53-thesis), (27.09.2010)

[15] Arthur R. M., Geselowitz D. B., Briller S. A., and Trost R. F. The path of the electrical center of the human heart determined from surface electrocardiograms. J. Electrocardiol., 4(1):29-33, 1971.

[16] Gulrajani R. M. and Mailloux G. M. A simulation study of the effects of torso inhomogeneities on electrocardiographic potentials, using realistic heart and torso models. Circ. Res., 52:45-56, 1983.

[17] Rudy Y. and Messinger-Rapport B. J. The inverse problem in electrocardiography: Solutions in terms of epicardial potentials. CRC Crit. Rev. in Biomed. Eng., 16:215-268, 1988.

[18] Hansen P. C. Rank-deficient and discrete ill-posed problems: Numerical aspects of linear inversion. 1998.

[19] Serinagaoglu Y., Brooks D.H., and MacLeod R.S. Bayesian solutions and performance analysis in bioelectric inverse problems. IEEE Trans. Biomed. Eng.,526:1009-1020, 2005. 
[20] Brooks D. H., Ahmad G. F., Macleod R. S., and Maratos G. M. Inverse electrocardiography by simultaneous imposition of multiple constraints. IEEE Trans. Biomed. Eng., 46-1:3-18, 1999.

[21] Berrier K.L., Sorensen D.C., and Khoury D.S. Solving the inverse problem of electrocardiography using a duncan and horn formulation of the kalman filter. IEEE Trans. Biomed. Eng., 51:507-515, 2004.

[22] Goussard Y., Joly D., and Savard P. Time-recursive solution to the inverse problem of electrocardiography. http://citeseer.ist.psu.edu/357941.html, (25.11.2010)

[23] El-Jakl J., Champagnat F., and Goussard Y. Time-space regularization of the inverse problem of electrocardiography. IEEE EMBC and CMBEC, pages 213-214, 1995.

[24] Joly D., Goussard Y., and Savard P. Time-recursive solution to the inverse problem of electrocardiography a model-based approach. Proc. IEEE-EMBS, pages 767-768, 1993.

[25] Aydin U., Serinagaoglu Y. "A Kalman Filter Based Approach to Reduce the Effects of Geometric Errors and the Measurement Noise in the Inverse ECG Problem", IFMBE Proceedings,2008.

[26] Jiang M., Xia L., Shou G., and Tang M. Combination of the lsqr method and a genetic algorithm for solving the electrocardiography inverse problem. Phys. Med. Biol., 52:1277-1294, 2007.

[27] Jiang M., Xia L., and Shou G. Noninvasive electrocardiographic imaging: Application of hybrid methods for solving the electrocardiography inverse problem. FIMH 2007, LNCS 4466, 4466:269-279, 2007.

[28] He B. and Wu D. Imaging and visualization of 3-d cardiac electrical activity. IEEE Trans. Inf. Tech. in Biomedicine, 5-3:181-186, 2001.

[29] Jiang, M., Xia, L., Shou, G., and Tang, M., Combination of the LSQR method and a genetic algorithm for solving the electrocardiography inverse problem, Phys. Med. Biol. 52 (2007) 1277-1294. 
[30] Jiang, M., Xia, L., and Shou, G., The Use of Genetic Algorithms for Solving Inverse Problem of Electrocardiography, EMBS Annual International Conference, 2006.

[31] Farina D., Jiang Y., Skipa O., D“ossel O., Kaltwasser C., and Bauer W. R. The use of the simulation results as a priori information to solve inverse problem of ecg for a patient. Computers in Cardiology, 32:571-574, 2005.

[32] Doessel O., Bauer W. R., Farina D., Kaltwasser C., and Skipa O. Imaging of bioelectric sources in the heart using a cellular automaton model. Proc. IEEE EMBS, pages 1067-1070, 2005.

[33] He B., Li G., and Zhang X. Noninvasive imaging of cardiac transmembrane potentials within three-dimensional myocardium by means of a realistic geometry anisotropic heart model. Phys. Med. Biol., 47:4063-4078, 2002.

[34] Nash M.P., Bradley C.P., Kardos A., Pullan A.J., and Paterson D.J. n experimental model to correlate simultaneous body surface and epicardial electropotential recordings in vivo. Chaos, Solitons and Fractals, 13:1735-1742, 2002.

[35] Oster H. and Tacardi B. Electrocardiographic imaging: Noninvasive characterization of intramural myocardial activation from inverse reconstructed epicardial potentials and electrograms. Circulation, 97:1496-1507, 1998

[36] Macleod R. S., Lux R. L., and Tacardi B. A possible mechanism for electrocardiographically silent changes in cardiac repolarization. J. Electrocardiol., 30:114-121, 1997.

[37] Barr R. C. and Spach M. S. Inverse calculation of qrs-t epicardial potentials from body surface potential distributions for normal and ectopic beats in the intact dog. Circ. Res., 42:661-675, 1978.

[38] Spach M. S., Barr R. C., Lanning C. F., and Tucek P. C. Origin of body surface qrs and $\mathrm{t}$ wave potentials from epicardial potential distributions in the intact chimpanzee. Circulation, 55:268-278, 1977. 
[39] Ramanathan C., Ghanem R. N., Jia P., Ryu K., and Rudy Y. Noninvasive electrocardiographic imaging for cardiac electrophysiology and arrhythmia. Nature Medicine, 2004.

[40] Colli Franzone P., Guerri L., Taccardi B., Viganotti C. "Finite element approximation of regularized solutions of the inverse potential problem of electrocardiography and applications to experimental data”. Calcolo, 22:91- 186, 1985.

[41] Wahba G., "Practical approximate solutions to linear operator equations when the data are noisy" SIAM J. Num. Anal. 14: 651-667, 1977.

[42] Golub G.H., Heat M., Wahba G. "Generalized cross-validation choosing a good ridge parameter" Technometrics 21:215-223, 1979.

[43] Hansen P. C. and OLeary D. P. "The use of the 1-curve in the regularization of discrete ill-posed problems". SIAM J. Sci. Comput., 14:1487-1503, 1993.

[44] Hansen P. C. Analysis of Discrete Ill-Posed Problems by Means of the L-Curve , SIAM Review, Vol. 34, No. 4, pp. 561-58, Dec 1992.

[45] Zhang Y., Ghodrati A., Brooks D.H., “An analytical comparison of three spatiotemporal regularization methods for dynamic linear inverse problems in a common statistical framework" Inverse Problems, vol. 21, pp. 357-382, 2005.

[46] Ghandi Ahmad F., Brooks Dana H., MacLeod Robert S., An Admissible Solution Approach to Inverse Electrocardiography. Annals of Biomedical Engineering Volume 26, Number 2, 278-292, DOI: 10.1114/1.56,

[47] Jameson A., "Solution of the equation $\mathrm{AX}+\mathrm{XA}=\mathrm{C}$ by inversion of an $\mathrm{M} \times \mathrm{M}$ or N × N matrix," SIAM J. Appl. Math., vol. 16, pp. 1020-1023, 1968.

[48] HERRERA F., LOZANO M, VERDEGAY J.L., Tackling RealCoded Genetic Algorithms: Operators and Tools for Behavioural Analysis Artificial Intelligence Review 12: 265-319, 1998.

[49] Kay S. M., Fundamentals of Statistical Signal Processing: Estimation Theory. Englewood Cliffs, NJ: Prentice-Hall, 1993. 
[50] Aster R., Borchers B., and Thurber T. Parameter Estimation and Inverse Problems , Academic Press, 2004.

[51] Greensite F. The temporal prior in bioelectromagnetic source imaging problems. IEEE Trans. Biomed. Eng., 50-10:1152-1159, 2003.

[52] Greensite F. A new treatment of the inverse problem of multivariate analysis. Inverse Problems, 18:363-379, 2002.

[53] Xiaoyun L., Xiaoling Z., and Xian X. Application of kalman filter in agricultural economic forecasting. IEEE Int. Conf. on Sys, Man, and Cybernetics, 3:2185-2189, 1996.

[54] Ennola K., Sarvala J., and Devai G. Modelling zooplankton population dynamics with the extended kalman filtering technique. Ecological Modelling 110, pp. 135-149, 1998, 110:135-149, 1998. 


\section{APPENDIX A}

\section{USING PRE/POST DIAGONALIZATION METHOD TO SOLVE MULTIPLE SPATIAL CONSTRAINTS}

Proceeding from equation 3.1 we start by setting

$$
\begin{aligned}
& \hat{\mathrm{x}}_{\lambda_{\mathrm{i}}}=\left(\mathrm{A}^{\mathrm{T}} \mathrm{A}+\sum_{\mathrm{i}=1}^{\mathrm{n}} \lambda_{\mathrm{i}}^{2} \mathrm{R}_{\mathrm{i}}^{\mathrm{T}} \mathrm{R}_{\mathrm{i}}\right)^{-1} \mathrm{~A}^{\mathrm{T}} \mathrm{y} \\
& \mathrm{P}=\mathrm{A}^{\mathrm{T}} \mathrm{A}+\sum_{\mathrm{i}=1}^{\mathrm{n}} \lambda_{\mathrm{i}}^{2} \mathrm{R}_{\mathrm{i}}^{\mathrm{T}} \mathrm{R}_{\mathrm{i}}
\end{aligned}
$$

Where $P x=Z$ and $Z=A^{T} Y$

Next we find the SVD of P and A

$\mathrm{A}=\mathrm{U}_{\mathrm{a}} \mathrm{S}_{\mathrm{a}} \mathrm{V}_{\mathrm{a}}^{*}$

$\mathrm{P}=\mathrm{U}_{\mathrm{p}} \mathrm{S}_{\mathrm{p}} \mathrm{V}_{\mathrm{p}}{ }^{*}$

The singular values of $\mathrm{P}$ are $\left(\sigma_{1}^{2}+\sum_{\mathrm{i}=1}^{\mathrm{n}} \lambda_{\mathrm{i}}^{2} \sigma_{\mathrm{R}}^{2}, \sigma_{2}^{2}+\sum_{\mathrm{i}=1}^{\mathrm{n}} \lambda_{\mathrm{i}}^{2} \sigma_{\mathrm{R}}^{2}+\ldots,+\sigma_{\mathrm{n}}^{2}+\sum_{\mathrm{i}=1}^{\mathrm{n}} \lambda_{\mathrm{i}}^{2} \sigma_{\mathrm{R}}^{2}\right)$ where $\sigma_{1}^{2}$ is the singular values of $\mathrm{A}$ and $\sigma_{R}^{2}$ are the singular values of the regularization matrixes [47].

$\mathrm{V}^{*}$ denotes the conjugate transpose of $\mathrm{V}$.

Let

$$
\mathrm{Q}=\mathrm{V}_{\mathrm{p}}{ }^{*} \mathrm{Z}
$$


And let

$\mathrm{w}_{\mathrm{ij}}=\frac{\mathrm{q}_{\mathrm{ij}}}{\sigma_{1}^{2}+\sum_{\mathrm{i}=1}^{\mathrm{n}} \lambda_{\mathrm{i}}^{2} \sigma_{\mathrm{R}}^{2}}$

We conclude with

$\widehat{\mathrm{x}}=\mathrm{V}_{\mathrm{p}} \mathrm{W}$

(a. 7) 


\section{APPENDIX B}

\section{USING PRE/ POST DIGONALIZATION METHOD TO SOLVE MULTIPLE SPATIO-TEMPORAL CONSTRAINTS}

The solution of multiple constraints approach with spatio-temporal constraints is:

$\hat{x}_{\lambda_{i} \eta_{\mathrm{j}}}=\left(\bar{A}^{T} \bar{A}+\sum_{\mathrm{i}=1}^{\mathrm{n}} \lambda_{\mathrm{i}}^{2} \overline{\mathrm{R}}_{\mathrm{i}}^{\mathrm{T}} \overline{\mathrm{R}}_{\mathrm{i}}+\sum_{j=1}^{k_{t}} \eta^{2}{ }_{\mathrm{j}} \bar{\Gamma}^{T}{ }_{j} \bar{\Gamma}_{j} \quad\right)^{-1} \bar{A}^{T} \bar{y}$

This equation can be written in the following form [20]:

$\mathrm{Px}+\mathrm{xS}=\mathrm{Z}$

where $Z=A^{T} Y$

$P=A^{T} A+\sum_{i=1}^{n} \lambda_{i}^{2} R_{i}^{T} R_{i}$

$\mathrm{S}=\sum_{\mathrm{j}}^{\mathrm{k}_{\mathrm{t}}} \eta_{\mathrm{i}} \Gamma_{\mathrm{i}}^{\mathrm{T}} \Gamma_{\mathrm{i}}$

As explained in the previous section we find the SVD values of A and P.

$$
\begin{aligned}
& \mathrm{A}=\mathrm{U}_{\mathrm{a}} \mathrm{S}_{\mathrm{a}} \mathrm{V}_{\mathrm{a}}{ }^{*} \\
& \mathrm{P}=\mathrm{U}_{\mathrm{p}} \mathrm{S}_{\mathrm{p}} \mathrm{V}_{\mathrm{p}}{ }^{*}
\end{aligned}
$$


The singular values of $\mathbf{P}$ are $\left(\sigma_{1}^{2}+\sum_{\mathrm{i}=1}^{\mathrm{n}} \lambda_{\mathrm{i}}^{2} \sigma_{\mathrm{R}}^{2}, \sigma_{2}^{2}+\sum_{\mathrm{i}=1}^{\mathrm{n}} \lambda_{\mathrm{i}}^{2} \sigma_{\mathrm{R}}^{2}+\ldots,+\sigma_{\mathrm{n}}^{2}+\right.$ $\sum_{\mathrm{i}=1}^{\mathrm{n}} \lambda_{\mathrm{i}}^{2} \sigma_{\mathrm{R}}^{2}$ ) where $\sigma_{1}^{2}$ is the singular values of $\mathrm{A}$ and $\sigma_{R}^{2}$ are the singular values of the regularization matrices [47].

$\mathrm{V}^{*}$ Denotes the conjugate transpose of $\mathrm{V}$.

$\mathrm{S}=\mathrm{U}_{\mathrm{S}} \mathrm{S}_{\mathrm{S}} \mathrm{V}_{\mathrm{S}}{ }^{*}$

Let

$\mathrm{Q}=\mathrm{V}_{\mathrm{p}}^{*} \mathrm{Z} \mathrm{V}_{\mathrm{T}}$

And let

$\mathrm{w}_{\mathrm{ij}}=\frac{\mathrm{q}_{\mathrm{ij}}}{\sigma_{1}^{2}+\sum_{\mathrm{i}=1}^{\mathrm{n}} \lambda_{\mathrm{i}}^{2} \sigma_{\mathrm{R}}^{2}+\sum_{\mathrm{j}=1}^{\mathrm{kt}} \eta_{\mathrm{j}}^{2} \sigma_{\Gamma}^{2}}$

We conclude with:

$\widehat{\mathrm{x}}=\mathrm{V}_{\mathrm{p}} \mathrm{WV}_{\mathrm{T}}^{\mathrm{T}}$ 
